\title{
COMPUTABLY BASED LOCALLY COMPACT SPACES
}

\author{
PAUL TAYLOR
}

University of Manchester

e-mail address: pt@cs.man.ac.uk

\begin{abstract}
ASD (Abstract Stone Duality) is a re-axiomatisation of general topology in which the topology on a space is treated, not as an infinitary lattice, but as an exponential object of the same category as the original space, with an associated lambda-calculus. In this paper, this is shown to be equivalent to a notion of computable basis for locally compact sober spaces or locales, involving a family of open subspaces and accompanying family of compact ones. This generalises Smyth's effectively given domains and Jung's strong proximity lattices. Part of the data for a basis is the inclusion relation of compact subspaces within open ones, which is formulated in locale theory as the way-below relation on a continuous lattice. The finitary properties of this relation are characterised here, including the Wilker condition for the cover of a compact space by two open ones. The real line is used as a running example, being closely related to Scott's domain of intervals. ASD does not use the category of sets, but the full subcategory of overt discrete objects plays this role; it is an arithmetic universe (pretopos with lists). In particular, we use this subcategory to translate computable bases for classical spaces into objects in the ASD calculus.
\end{abstract}

\section{Contents}

Introduction

1. Locally compact sober spaces

2. Locally compact locales

3. Axioms for abstract Stone duality

4. Sets, unions and bases

5. Compact subspaces

6. Effective bases

7. Sigma-split subobjects

8. Every definable object has a basis

9. Basic corollaries

10. Primes and nuclei

11. The way-below relation

2000 ACM Subject Classification: F.4.1.

2000 Mathematics Subject Classification: 54D45, 03D45 (Primary), 06B35, 54D30, 68N18 (Secondary).

Key words and phrases: abstract Stone duality, locally compact, basis for topology, effectively presented, way below relation, continuous lattice, Sigma-split subspace. 
12. Domain theory in ASD 47

13. The lattice basis on $\Sigma^{N}$

14. From the basis to the space

15. The points of the new space 57

16. Morphisms as matrices 60

17. Relating the classical and term models $\quad 64$

References $\quad 68$

Acknowledgements 70

\section{INTRODUCTION}

A locally compact space is one in which there is a good interaction of open and compact subspaces. In this paper we shall show how this interaction can be captured abstractly, establishing an equivalence with a new recursive account of topology.

We shall consider the following formulations of local compactness:

(a) open and compact subspaces in a traditional sober topological space (Section 11);

(b) disjoint open subspaces in a compact Hausdorff (regular) space (Example 6.11); and

(c) the way-below relation $\ll$ in a continuous distributive lattice (Section 2).

In the categories of locally compact sober spaces and locales, the topology (lattice of open subspaces) of $X$, considered as another space or locale equipped with the Scott topology, is the exponential $\Sigma^{X}$, where $\Sigma$ is the Sierpiński space. We shall show how the three classical formulations above give rise to the following abstract structure in this category:

(d) families of terms $\beta^{n}: \Sigma^{X}$ and $A_{n}: \Sigma^{\Sigma^{X}}$ with a basis expansion (Section 6)

$$
\phi x \Leftrightarrow \exists n . A_{n} \phi \wedge \beta^{n} x
$$

(e) a $\Sigma$-split inclusion $i: X \succ \Sigma^{N}$ and $I: \Sigma^{X} \triangleleft \Sigma^{\Sigma^{N}}$ with $\Sigma^{i} \cdot I=\mathrm{id}_{\Sigma^{X}}$ (Section 7);

(f) an idempotent $\mathcal{E} \equiv I \cdot \Sigma^{i}$ on $\Sigma^{\Sigma^{N}}$ (Section [10);

(g) an abstract way-below relation $n \preccurlyeq m \equiv A_{n} \beta^{m}$ on $N$ (Section 11); and

(h) an Eilenberg-Moore algebra for the monad arising from the adjunction $\Sigma^{(-)} \dashv \Sigma^{(-)}[\mathrm{B}$.

We will give complete axiomatisations of each of these structures, and define the translations amongst them, doing this in a new $\lambda$-calculus (Abstract Stone Duality). We consider several special cases, in particular the real line, the interval domain (Definition [7.10), continuous dcpos and various kinds of domains (Section 12).

It may help you to find your way around this lengthy paper if I explain that Section 6 was the first to be written. It shows how the classical notions of locally compact space and locale can be expressed in a $\lambda$-calculus.

However, the present paper was the first to put this calculus to work to do topology. The previous ones $[\mathrm{A}, \mathrm{B}, \mathrm{C}]$ had examined components of the theory as abstract categorical ideas, without being aware of how many (in fact, few) more such components would be needed to build a logically complete account of topology. Sections 34 and 70 discuss the way in which these components go together to make a viable theory. This discussion has, 
in fact, spilled over into several additional papers, in particular $\mathrm{E}, \mathrm{H}$. If you would prefer to read a dry summary of the axioms in a "user manual" style, please see [I]

Once all this structure is in place, Section 11] begins to take it apart again, characterising the finitary properties of the way below relation in an abstract way. This corresponds to the concrete treatment in Sections 1 and 2. Sections 13 16] construct an object of the ASD calculus from any such abstract relation (with a "practice run" for domains in Section [12) and the final section completes the circle with the traditional theory.

\section{LOCALLY COMPACT SOBER SPACES}

Definition 1.1. The traditional definition of local compactness was generalised from Hausdorff to sober spaces in [HM81, p. 211]: Whenever a point is contained in an open subspace $(x \in V)$, there is a compact subspace $K$ and an open one $U$ such that $x \in U \subset K \subset V$.

It is an easy exercise in the "finite open sub-cover" definition of compactness to replace the point $x$ by another compact subspace:

Lemma 1.2. Let $L \subset V \subset X$ be compact and open subspaces of a locally compact space. Then there are $L \subset U \subset K \subset V \subset X$ with $U$ open and $K$ compact.

We call this result the interpolation property. Alternating inclusions of open and compact subspaces like this will be very common in this paper, and compact subspaces will be more important than points.

Notation 1.3. We write $U \nVdash V$ and $K \preccurlyeq L$ if there is such an interpolating compact or open subspace, respectively. The second version, in which $K \supset L$, follows the usage of [HM81, JS96], $c f$. our Theorem 5.18.

Now consider what we might mean by a computably defined locally compact space.

Suppose that you have some computational representation of a space. It can only encode some of the points and open and compact subspaces, since in classical topology there are uncountably many of them in any interesting case. Hence your "space" cannot be literally sober, or have arbitrary unions of open subspaces. We understand the intended space to be the corresponding sober one, in which arbitrary unions of opens have also been adjoined. Those points, open and compact subspaces that have codes are called basic.

Example 1.4. A computable definition of $\mathbb{R}$ as a locally compact space might have

(a) as basic points, (encodings of) the rationals;

(b) as basic open subspaces, the (names of) open intervals $(q \pm \epsilon) \equiv(q-\epsilon, q+\epsilon) \equiv$ $\{x|| x-q \mid<\epsilon\}$ with rational or infinite endpoints; and

(c) as basic compact subspaces, the closed intervals $[q \pm \delta] \equiv[q-\delta, q+\delta] \equiv\{x|| x-q \mid \leq \delta\}$ with finite rational endpoints.

Notice that both open and closed intervals are used, although treatments of exact real arithmetic often just use one or the other, $c f$. Example 17.12 Also, by "rationals" we might actually mean all pairs $p / q$ with integers $p$ and $q \neq 0$, or the dyadic rationals $p / 2^{n}$, or continued fractions, or whatever our favourite countable dense set of reals may be. Unlike Dedekind cuts, this example readily generalises: for $\mathbb{R}^{3}$ we use open and closed cuboids whose vertices have rational co-ordinates (or, better, a system based on close packing of spheres CS88). 
In the Example, the intersection of two basic opens is again basic, but for technical reasons we shall also need to extend the families to include finite unions of open (respectively, compact) intervals or cuboids. It's an exercise that's a little too complicated to be called algebra, but easy programming, to test inclusion and compute the representations of such unions and intersections.

Definition 1.5. A computably based locally compact space consists of a set of codes for basic "points", "open" and "compact" subspaces, together with an interpretation of these codes in a locally compact sober space. We require of the space that every open subspace be a union of basic ones. We also want to be able to compute

(a) codes (that we shall just call 0 and 1) for the empty set and the entire space, considered as open and compact subspaces (if, that is, the entire space is in fact compact);

(b) codes for the union and intersection of two open subspaces, and for the union of two compact ones, given their codes (we write + and $\star$ instead of $\cup$ and $\cap$ for these binary operations, to emphasise that they act on codes, rather than on the subspaces that the codes name);

(c) whether a particular representable point belongs to a particular basic open subspace, given their codes; but we only need a positive answer to this question if there is one, as failure of the property is indicated by non-termination;

(d) more generally, whether an open subspace includes a compact one, given their codes;

(e) codes for $U$ and $K$ such that $L \subset U \subset K \subset V$, given codes for $L \subset V$ as above.

(f) In fact, we shall require the basic compact and open subspaces to come in pairs, with $U^{n} \subset K^{n}$ as in [JS96], where the superscript $n$ names the pair, and we also need part

(e) to yield such a pair as the interpolant.

Extensional equivalence of computable functions is not captured within the strength of the logic that we wish to study. So, for the "computations" above, we mean a particular program to be specified - at least up to provable equivalence, which means that we don't have to nominate a programming language.

Definition 1.6. A computably continuous function between such spaces is a continuous function $f: X \rightarrow Y$ between the topological spaces themselves, for which the binary relation

$$
f K^{m} \subset U^{n}
$$

(between the codes $(n, m)$ for a compact subspace $K^{m} \subset X$ and an open one $U^{n} \subset Y$ ) is recursively enumerable, $c f$. part (c) of the previous definition.

In particular, computably equivalent bases for the same space are those for which the identities in both directions are computably continuous functions. This means that the relations $K^{n} \subset U^{m}$ and $K^{m} \subset U^{n}$ between $n$ and $m$ are recursively enumerable. For example, whilst there are several choices for the "rationals" and intervals in Example 1.4. all of the reasonable ones are computably equivalent.

Remark 1.7. It is out of place in the definition of something computable to specify a topological space: this was only included to guarantee topological consistency of the computations of union, intersection, containment and interpolation. This is also the reason why the codes for basic points played no actual role in Definition 1.5 - there needn't be any of them. Since we said that the spaces are sober, continuous functions are determined entirely by their effect on basic open and compact subspaces, as they are in locale theory. Neither 
the (optional) basic points, nor the computable ones that we recover in Section 15, nor the uncountably many points of the classical theory, are actually needed to specify continuous functions, or prove topological properties such as compactness.

At the technical level, therefore, what we call a computably based locally compact "space" is really just a system of codes and programs acting on them, for which there exists a topological space in which these codes may be interpreted as compact and open subspaces. Likewise, a computably "continuous function" is just an equivalence class of programs, for which there exists a continuous function that agrees with the program in the way that we have said.

The particular way in which computations are set up is of course a matter for discussion: I still have to convince you of the merits of my way, in comparison to the many other ways that have been used to calculate in $\mathbb{R}^{\mathbf{n}}$ over the millennia, and of course any theoretical picture is subject to technical optimisation. However, these other ways share the same pattern, in that they have a technical definition that is (a) used for applied computation but (b) justified by the existence of an interpretation in some "pure" theory of (Euclidean) space. The pure theory is not the computation, at any rate if it is based on twentieth century topology. So the practical situation is illustrated by Example 1.4 we have a classical definition of a topological space, equipped with a basis that is defined in some conventional way, and which we want to use to obtain values in the space. So long as the above features of the basis are computable, the classical space guarantees the consistency conditions.

In Section 11 we shall formulate this consistency in terms of finitary conditions on the transformations of encodings themselves, eliminating the topological space, i.e. we prove necessity of the abstract conditions. Then in Section [14 we prove sufficiency, i.e. that any such encoding (such as the one for $\mathbb{R}$ above) satisfying these consistency requirements does define a locally compact sober space, and this is unique up to homeomorphism.

This construction is done, not in traditional topology itself, which is not computable, but in a $\lambda$-calculus. Therefore, the construction imports the classical data into the computational world (Section 177). But again there is a distinction between the technical and philosophical meaning: whilst our theory (ASD) starts from (and is reducible back to) computational foundations, it adapts these to give a new account of the mathematical theory that is ultimately intended to be usable by pure mathematicians in place of the old one.

Remark 1.8. The operations $\star$ and + become $\cap$ and $\cup$ when we interpret them via the basic open subspaces that they encode. Amongst the abstract consistency requirements, therefore, we would expect $(0,1,+, \star)$ to define a distributive lattice.

However, we have only asked for the ability to test inclusion of a compact subspace in an open one, not inclusion or equality of two open or two compact subspaces, nor of an open subspace in a compact one (the inclusion $U^{n} \subset K^{n}$ is given, not tested). Even if these happen to be possible, it is computationally quite reasonable for different codes to denote the same subspace, but for this fact to be potentially undecidable.

On the other hand, as we want to stress the computable aspect of the names of basic subspaces, we shall often represent + and $\star$ as concatenations of lists. This clumsiness actually serves an expository purpose, keeping this "imposed" structure on codes separate in our minds from the "intrinsic" structure in the topology on $X$. (We shall regard this topology as another space.) If we used the notation and equations of a distributive lattice for the set $N$ of codes, it would be all too easy to lapse into confusing it with the actual 
topology on the space. This would in fact make logical assumptions that amount to a solution of the Halting Problem (or worse).

The topological information is actually contained, not in the quasi-lattice structure $(0,1,+, \star)$ on $N$, but in the inclusion relation between compact and open subspaces. This satisfies some easily verified properties:

Lemma 1.9. $\emptyset \subset V$,

$$
\frac{K \subset L \subset U \subset V}{K \subset V} \quad \frac{K \subset V \quad L \subset V}{K \cup L \subset V} \quad \frac{K \subset U \quad K \subset V}{K \subset U \cap V}
$$

Finally, there is a property similar to Lemma 1.2 that concerns binary unions. Easy enough though this property may be to prove - when you see it — it is not something whose significance one would identify in advance. Various forms of it were originally studied by Peter Wilker Wil70].

Lemma 1.10. Let $K$ be a compact subspace covered by two open subspaces of a locally compact sober space $X$, that is, $K \subset U \cup V$. Then there are compact subspaces $L$ and $M$ and open ones $U^{\prime}$ and $V^{\prime}$ such that

$$
K \subset U^{\prime} \cup V^{\prime} \quad U^{\prime} \subset L \subset U \text { and } V^{\prime} \subset M \subset V .
$$

Proof. Classically, $K \backslash V$ is a closed subspace of a compact space, and is therefore compact too, whilst $K \backslash V \subset U$, so by the interpolation property (Lemma 1.2) we have

$$
K \backslash V \subset U^{\prime} \subset L \subset U \subset X
$$

for some $U^{\prime}$ open and $L$ compact. Then $K \backslash U^{\prime} \subset K \backslash(K \backslash V) \subset V$ so

$$
K \backslash U^{\prime} \subset V^{\prime} \subset M \subset V \subset X
$$

for some $V^{\prime}$ open and $M$ compact. Finally, $K=\left(K \cap U^{\prime}\right) \cup\left(K \backslash U^{\prime}\right) \subset U^{\prime} \cup V^{\prime}$.

We didn't mention the intersection of two compact subspaces in Definition 1.5, because there are spaces in which this need not be compact.

Definition 1.11. A locally compact sober space is called stably locally compact if the whole space is compact and the intersection of any two compact subspaces is again compact.

Examples 1.12. (a) Consider two copies of the real unit interval $[0,1]$ identified on their interiors (or, if you prefer, an interval with duplicated endpoints). Then the two copies of the interval are compact subspaces, but their intersection is not [Kel55, Problem 5 B(a)].

(b) A combinatory algebra has constants $k$ and $s$ and a (non-associative) binary operation - such that $(k \cdot x) \cdot y=x$ and $(((s \cdot x) \cdot y) \cdot z)=(x \cdot z) \cdot(y \cdot z)$. In the free such algebra $A$, terms can be enumerated, and proved equal, using these rules. But, since this structure can be used to encode computation [Bar81, proving inequality in $A$ is like solving the Halting problem. (Similar unsolvable word problems can also be set up in other algebraic theories, such as groups.)

Hence $A$ is discrete but not Hausdorff.

Since it is discrete, its compact subspaces are the Kuratowski-finite ones, i.e. those that can be listed, possibly with repetition. In particular, singletons $\{x\}$ are open and compact, but not closed. However, the intersection $\{x\} \cap\{y\}$ is compact (Kuratowskifinite, listable) iff it is either empty $(x \neq y)$ or a singleton $(x=y)$, i.e. iff equality is decidable, which it isn't. 


\section{Locally COMPaCt LOCALES}

Points disappeared from the discussion right at the beginning, and we saw in Example 1.4 that it is easier to specify $\mathbb{R}$ with the Euclidean topology using open and compact subspaces than using open subspaces and points. Arguably, topology should be axiomatised in this way, just as traditional geometry was axiomatised in terms of lines and circles that were entities in themselves, rather than being sets of points.

Locale theory reduces the description further, to one involving open subspaces alone. To do this for locally compact spaces, we must represent compact subspaces in terms of their

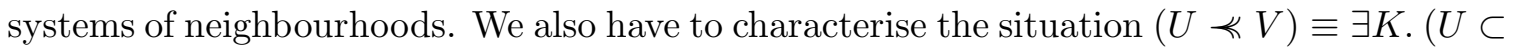
$K \subset V)$. We shall see that the way in which this is done for locales is subtly different from that for spaces, especially in the non-stably locally compact case (Remark 6.17).

Definition 2.1. Let $L$ be a complete lattice.

(a) A family $\left(\psi_{s}\right) \subset L$ is called directed ${ }^{1}$ if it is inhabited, and whenever $\psi_{r}$ and $\psi_{s}$ belong to the family, there is some $\psi_{t} \geq \psi_{r}, \psi_{s}$. The join of the family is written $\bigvee \psi_{s}$.

(b) Now, for $\beta, \phi \in L$, we write $\beta \ll \phi$ (way-below) if, whenever $\phi \leq \bigvee_{s} \psi_{s}$, there is already some $s$ for which $\beta \leq \psi_{s}$. (So $\beta \ll \phi$ implies $\beta \leq \phi$.)

(c) Then $L$ is a continuous lattice $\mathrm{GHK}^{+} 80$, HM81] if, for all $\phi \in L, \quad \phi=\bigvee\{\beta \mid \beta \ll \phi\}$.

Proposition 2.2. The topology of any locally compact space is a distributive continuous lattice, in which $U \ll V$ iff $U \ll V$ [HM81, p. 212].

Proof. $\quad U \ll V$ implies $U \ll V$ by compactness of $K$ with $U \subset K \subset V$, and

$$
V=\bigcup\{W \mid W \prec V\}
$$

by Definition 1.1. This union is directed by Lemma 1.9 so it may be used in the definition of $U \ll V$, giving $U \subset W \prec V$ for some $W$, but then $U \prec V$ too. Hence $U \ll V$ iff $U \ll V$, but notice that the proof does not supply an interpolating compact subspace $U \subset K \subset V$.

Conversely, every distributive continuous lattice is the lattice of open subspaces of some locally compact sober space. However, this result relies on the axiom of choice, and even then it is not a trivial matter to prove it (Remark 17.6 [HL78]).

Definition 1.5 for spaces has a simpler analogue for locales, since it's all lattice theory.

Definition 2.3. A computable basis $(N, 0,1,+, \star, \preccurlyeq)$ for a continuous distributive lattice $L$ is a set $N$ with constants $0,1 \in N$, computable binary operations $+, \star: N \times N \rightarrow N$, a recursively enumerable binary relation $\nprec$ and an interpretation $\beta^{(-)}: N \rightarrow L$ that takes $(0,1,+, \star)$ to the lattice structure in $L$, such that $n \ll m$ iff $\beta^{n} \ll \beta^{m}$ and

$$
\text { for each } \phi \in L, \quad \phi=\bigvee\left\{\beta^{n} \mid \beta^{n} \ll \phi\right\} \text {. }
$$

If $L_{1}$ and $L_{2}$ have bases $\left(\beta^{m}\right)$ and $\left(\gamma^{n}\right)$ then $H: L_{2} \rightarrow L_{1}$ is a computable frame homomorphism if $H$ preserves $\top, \wedge$ and $\bigvee$, and the relation $\left(\beta^{m} \ll H \gamma^{n}\right)$ is recursively enumerable. The interested reader may like to translate the abstract conditions on this relation that are set out in Section [16] into locale theory, and thereby recover the frame homomorphism $H$.

Once again we seek to remove the locale or continuous lattice from the definition, this time with the goal of eliminating the infinitary joins in favour of finitary properties of the

\footnotetext{
${ }^{1}$ The letter $s$ stands for semilattice, but see Definition 4.13
} 
way-below relation. Of course, since « was itself defined using directed joins, in Section 11 it will have to be replaced by an abstract relation $\preccurlyeq$. This will satisfy axioms based on the following properties, which are the analogues of Lemmas [1.2, 1.9] and 1.10.

Lemma 2.4. If $\beta^{\prime} \leq \beta \ll \phi \leq \phi^{\prime}$ then $\beta^{\prime} \ll \phi^{\prime}$.

Lemma 2.5. The relation $\ll$ is transitive and interpolative: if $\alpha \ll \beta \ll \gamma$ then $\alpha \ll \gamma$, and conversely given $\alpha \ll \gamma$, there is some $\beta$ with $\alpha \ll \beta \ll \gamma$.

Lemma 2.6. $\perp \ll \phi$, and if $\alpha \ll \phi$ and $\beta \ll \phi$ then $(\alpha \vee \beta) \ll \phi$.

The Wilker property in Lemma 1.10 used excluded middle, but its analogue for continuous lattices is both intuitionistic and very simple: it follows from the observation that binary joins distribute over joins of inhabited, and in particular directed, families.

Lemma 2.7. In any continuous lattice, if $\alpha \ll \beta \vee \gamma$ then $\alpha \ll \beta^{\prime} \vee \gamma^{\prime}$ for some $\beta^{\prime} \ll \beta$ and $\gamma^{\prime} \ll \gamma$.

Proof. Since any directed set is inhabited,

$$
\beta \vee \gamma=\bigvee\left\{\beta^{\prime} \vee \gamma \mid \beta^{\prime} \ll \beta\right\}=\bigvee\left\{\beta^{\prime} \vee \gamma^{\prime} \mid \beta^{\prime} \ll \beta, \gamma^{\prime} \ll \gamma\right\} .
$$

Then if $\alpha \ll \beta \vee \gamma$, we have $\alpha \ll \beta^{\prime} \vee \gamma^{\prime}$ for some term in this join.

The relationship between $\ll$ and $\wedge$ is more subtle.

Lemma 2.8. If $\alpha \ll \beta \ll \phi$ and $\beta \ll \psi$ then $\alpha \ll \phi \wedge \psi$.

Definition 2.9. A stably locally compact locale is one in which $T \ll \top$, and if $\beta \ll \phi$ and $\beta \ll \psi$ then $\beta \ll \phi \wedge \psi$.

Examples 1.12 can be adapted to yield locally compact locales that are not stably locally compact, and therefore only obey the weaker rule in the Lemma.

Remark 2.10. Stably locally compact objects enjoy many superior properties, illustrating the duality between compact and open subspaces. Jung and Sünderhauf [JS96] set out "consistency conditions" for them that are similar to ours, except that they choose to make $(0,1,+, \star)$ a genuine ("strong proximity") lattice.

However, as Examples 1.12 illustrated, not all of the locally compact objects that we wish to consider are stably so, either in geometric topology or recursion theory. Most obviously for the former, in $\mathbb{R}$, the whole space (the trivial intersection) is not compact.

Another reason why we consider the more general situation is that it corresponds to the monadic Axiom 3.3(c) that was the fundamental idea behind the research programme of which this paper is a part. This correspondence, which has no counterpart in [JS96], is the main technical goal of this paper; the consistency conditions are just an intermediate step.

We shall see at the end of Section [6] that the non-stable situation also highlights an interesting difference (separate from the usually mentioned ones of constructivity) between locales and sober spaces as ways of presenting topological information.

Applying G.H. Hardy's test Har40, we may wonder which of the stable and non-stable theories is "beautiful" and which is "ugly". My suspicion is that stably locally compact spaces and the "relational" morphisms that they describe (JKM01, and our [9.11, 12.13, 
15.5 and [16.15) play a different role in the bigger picture, whilst we are right to study "functional" morphisms in the non-stable case.

The logic that we use is a very weak computational one, but on closer examination, we see that a great deal of the work that has been done in domain theory, using many notions of "basis" or "information system" could actually be formulated in such a logic.

\section{Axioms for abstract Stone Duality}

In this paper we develop a computable account of locally compact sober spaces and locales, but using a $\lambda$-calculus in place of the usual infinitary lattice theory, which conflicts with computable ideas. This calculus, called Abstract Stone Duality, exploits the fact that, for any such space $X$, its lattice of open subspaces provides the exponential $\Sigma^{X}$ in the category. Here $\Sigma$ is the Sierpiński space (which, classically, has one open and one closed point), and the lattice $\Sigma^{X}$ is equipped with the Scott topology. Its relationship to the "consistency conditions" in the previous sections and in JJS96 will be examined in Sections 11, 16

In this section and the next we present the axiomatisation of ASD in a reflective fashion that is intended to examine the reasons for our choices. For a straightforward summary of the calculus intended for applications, see [I] instead.

Remark 3.1. We are primarily interested in two particular models of the calculus:

(a) as a source of topological intuition, the classical ones, namely the categories of locally compact sober spaces (LKSp) and of locally compact locales (LKLoc), maybe over an elementary topos other than Set;

(b) for computation, the term model.

The classical side has a wealth of concepts motivated by geometry and analysis, but its traditional foundations are logically very strong, being able to define many functions that are neither continuous nor computable, besides many other famous pathologies. Having created such a wild theory, we have to rein it back in again, with a double bridle. The topological bridle is constructed with infinitary lattice theory, whilst in recursion theory we are reduced to using Gödel numberings of manipulations of codes for basic elements. Abstract Stone duality avoids all of this by only introducing computably continuous functions in the first place. (We pay a logical price for this in not being able to define objects and functions anything like so readily as in set theory.)

On the other hand, logically motivated discussions often read the foundational aspects more literally than their topological authors ever intended. They are so bound up in their own questions of what constitutes "constructivity" that they lose sight of the conceptual structure behind the mathematics itself. For one example, in several constructive approaches to topology, the closed real interval fails to be compact. For another, whilst the mathematician in hot pursuit of a proof typically postulates a least counterexample, in order to rule it out, it is impertinent of the logician to emphasise that this uses excluded middle, since proofs of this kind (once found) can very often be recast in terms of the induction scheme Tay99, Section 2.5]. As a result, foundational work often fails to reach "ground level" in the intended construction.

The lesson that we draw from this is that logic and topology readily drift apart, if ever we let go of either of them for just one moment. So we have to tell their stories in parallel. This means that we must often make do with rough-and-ready versions of parts of one, in 
order to make progress with the other. For example, we use concepts such as compactness to motivate our $\lambda$-calculus, which itself underlies the technical machinery that eventually justifies the correspondence with traditional topology and the use of its language. We shall make a point of explaining how each step (Definition, Lemma, etc.) of the new argument corresponds to some idea in traditional topology.

This means that you will have to be prepared for some sudden switches between contexts. This paper is highly experimental. It introduces $\lambda$-calculus formulations of topological concepts and arguments, which it has to try out in the traditional categories for topology, even though these are defective for the purpose. When we are sure that we have all of the axioms in place (which was certainly not the case before this investigation began, but is close to being so after it) the best way to handle the relationship will not be to interpret ASD in locale theory, but vice versa $[\mathrm{H}$.

In this section we have to set up some logical structure to which the topologically minded reader may prefer to return after first reading Section [6 We catch up on some of the justifications in Sections 9 and 10, for example characterising our morphisms in terms of preservation of meets and joins. In this section too, we interleave the axioms and definitions of the $\lambda$-calculus with the technical issues that they are intended to handle.

Remark 3.2. Recall first the universal properties of the Sierpiński space, $\Sigma$. Any open subspace $U \subset X$ is classified by a continuous function $\phi: X \rightarrow \Sigma$, in the sense that $U=\phi^{-1}(\top)$, where $T$ is the open point of $\Sigma$, and $\phi$ is unique with this property. This is summed up by the pullback diagram

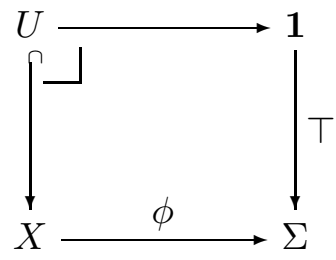

In our calculus, we shall use the $\lambda$-term $\phi: \Sigma^{X}$ instead of the subset $U \subset X$. A similar diagram, using the closed point $\perp \in \Sigma$ instead of $T$, classifies (or, as we shall say, coclassifies) the closed subspace, that, classically, is complementary to $U$. We follow topology rather than logic in retaining the bijection between open and closed subspaces, even though they are not actually complementary in the sense of constructive set theory. This is because it is not sets but topological spaces that we wish to capture.

Unions and intersections of open and closed subspaces make the topology $\Sigma^{X}$, and in particular the object $\Sigma$, into distributive lattices - honest ones now, not consisting of codes as in Remark 1.8. We therefore have to axiomatise the exponential and the lattice structure.

Axiom 3.3. The category $\mathcal{S}$ of "spaces" has finite products and an object $\Sigma$ of which all exponentials $\Sigma^{X}$ exist. Then the adjunction $\Sigma^{(-)} \dashv \Sigma^{(-)}$that relates $\mathcal{S}^{\text {op }}$ to $\mathcal{S}$ is to be monadic. This categorical statement has an associated symbolic form, consisting of

(a) the simply typed $\lambda$-calculus, except that we may only introduce types of the form $\Sigma^{X \times Y \times \cdots}$ (or $X \rightarrow Y \rightarrow \cdots \rightarrow \Sigma$ if you prefer), and therefore $\lambda$-abstractions whose bodies already have such types;

(b) an additional term-forming operation, focus, which may only be applied to a term $P$ of type $\Sigma^{\Sigma^{X}}$ that satisfies a certain primality equation capturing the situation $P=\lambda \phi . \phi a$, and then focus $P=a$; the use of focus makes the space $X$ sober $\mathrm{A}$; 
(c) and an additional type-forming operation (with associated term calculus) that provides a formal subspace $i: X \equiv\{Y \mid E\} \longmapsto Y$ with $I: \Sigma^{X} \triangleleft \Sigma^{Y}$ such that $\Sigma^{i} \cdot I=\operatorname{id}_{\Sigma}$ given an endomorphism $E: \Sigma^{Y} \rightarrow \Sigma^{Y}$ satisfying a certain equation, and then $E=I \cdot \Sigma^{I}$; members of the formal subspace are then the admissible terms $a: Y$, i.e. those satisfying yet another equation, for which we may then introduce admit $a: X[\mathrm{~B}]$.

Remark 3.4. The equations required in parts (b) and (c) can each be formulated in two different ways: an abstract one that states the underlying monadic idea in $\lambda$-notation, and a lattice-theoretic one that takes advantage of the additional "topological" structure of the calculus. One of our many tasks in this paper (Section 10) is to prove the equivalence of these formulations.

In (b), we shall find that $P: \Sigma^{\Sigma^{X}}$ is prime iff it is a lattice homomorphism:

$$
P \top \Leftrightarrow \top \quad P \perp \Leftrightarrow \perp \quad P(\phi \wedge \psi) \Leftrightarrow P \phi \wedge P \psi \quad \text { and } \quad P(\phi \vee \psi) \Leftrightarrow P \phi \vee P \psi,
$$

whilst for (c), the term $E$ is of the form $I \cdot \Sigma^{i}$ iff

$$
E(\phi \wedge \psi)=E(E \phi \wedge E \psi) \text { and } E(\phi \vee \psi)=E(E \phi \vee E \psi) \text {. }
$$

We call such a term $E$ a nucleus, shamelessly appropriating this word from locale theory, in which a nucleus is a monotone endofunction $j: L \rightarrow L$ of a frame such that id $\leq j=j \cdot j$. A nucleus $E$ in our sense must be Scott continuous, but need not be order-related to the identity, but the senses agree when $E \geq$ id and $j$ is Scott continuous.

We discuss $\Sigma$-split subspaces in Section 7 and give the admissibility equation in Definition 12.8, which is where we first use it in this paper.

Remark 3.5. We have said that we are interested in the term model of the calculus. Now, this is powerful enough to encode computations, because domain theory can be developed in it (Section 12) and programming languages such as Plotkin's PCF can be interpreted using standard techniques of denotational semantics [Plo77. This means that we cannot hope to give an explicit description of the term model: the best we can do is to indicate how the topological features of ASD can be "compiled out", to yield programs in some recognisable programming language.

In [B, $\S \S 9-11]$, arbitrarily complicated combinations of $\Sigma$-split subspaces and exponentials are reduced to a single $\Sigma$-split subspace of a (still complicated) exponential. In this paper (Section 8) we shall reduce this even further, to a $\Sigma$-split subspace of $\Sigma^{N}$, showing that this description is equivalent to giving an $N$-indexed basis for $X$. The terms also have a corresponding normalisation.

This leaves focus, the quantifiers, recursion etc. on $\Sigma^{\mathbb{N}}$. The idea of focus is that it recovers a point of a space from its Scott-open prime filter of neighbourhoods, which can be characterised using either infinitary lattice theory [A, §2] or the $\lambda$-calculus arising from the monad [A, §4]. In this calculus, focus is pushed to the outside of any term, and is shown to be redundant at all objects except $\mathbb{N}[\mathrm{A}, \S 8]$.

Using the (abstract) lattice structure and primitive recursion, focus on $\mathbb{N}$ is interdefinable with definition by description [․, $\$ \S 9-10]$. General recursion can also be defined. Finally, [A, §11] sketches how everything apart from disjunction and recursion strongly normalises, to what is essentially a PROLOG clause. Restoring those two features yields a (parallel) Prolog program. On the other hand, normalising the $\lambda$-applications may not be such a good idea, so it's better to think of ASD terms as parallel $\lambda$-PROLOG programs.

The free model of the calculus therefore satisfies the Church-Turing thesis (i.e. it is equivalent to thousands of other ways of writing programs) so we do not need to introduce 
Kleene-style notation with Gödel numbers in order to justify calling it a computable account of topology. In particular, the "computations" referred to in Definitions 1.5, 1.6] and 2.3 may be defined by terms of our calculus.

Axiom 3.6. Returning to the Sierpiński space itself, $(\Sigma, \top, \perp, \wedge, \vee)$ is an internal distributive lattice in $\mathcal{S}$, which also satisfies the Phoa principle,

$$
F: \Sigma^{\Sigma}, \sigma: \Sigma \vdash F \sigma \Leftrightarrow F \perp \vee \sigma \wedge F \top \text {. }
$$

This equation (which is bracketable either way, by distributivity) is used to ensure that terms of type $\Sigma^{X}$ yield data for the open or closed subspace of $X$, as required by the monadic axiom [C, Sections 2-3], and hence the pullback in Remark [3.2. It thereby enforces the bijective correspondences amongst open and closed subspaces and terms of type $\Sigma^{X}$. We shall assert an "infinitary" generalisation of the Phoa principle shortly.

Definition 3.7. The lattice structure on $\Sigma$ and $\Sigma^{X}$ defines an intrinsic order, $\leq$, where $\phi \leq \psi$ iff $\phi=\phi \wedge \psi$ iff $\phi \vee \psi=\psi$. In the case of terms of type $\Sigma$ (which we call propositions), we shall write $\Rightarrow$ and $\Leftrightarrow$ instead of $\leq$ and $=$. The order is inherited by other objects:

$$
\Gamma \vdash a \leq b: X \quad \text { if } \Gamma \vdash\left(\lambda \phi: \Sigma^{X} . \phi a\right) \leq(\lambda \phi . \phi b): \Sigma^{\Sigma^{X}} .
$$

There are other ways of defining an order, but sobriety and the Phoa principle make them equivalent, and also say that all maps are monotone [C, Section 5].

In LKLoc this order on hom-sets arises from the order on the objects, considered as lattices, whilst in LKSp it is the specialisation order,

$$
x \leq y \equiv(\forall U \subset X \text { open. } x \in U \Rightarrow y \in U) \equiv x \in \overline{\{y\}},
$$

where $\overline{\{y\}}$ is the smallest closed subspace containing $y$.

Lemma 3.8. Let $i: U \longrightarrow X$ and $j: C \longmapsto X$ be the open and closed subspaces (co)classified by $\phi: \Sigma^{X}$. Then, with respect to the intrinsic orders on $\Sigma$ and $\Sigma^{X}$, there are adjoints

$$
\exists_{i} \dashv \Sigma^{i} \quad \text { and } \quad \Sigma^{j} \dashv \forall_{j}
$$

that behave like quantifiers [C] Section 3]. (Note the different tails on the arrows.)

Remark 3.9. The order $\leq$ on $\Sigma^{X}$ presents an important problem for the axiomatisation of the join in Definition 2.1(c)

$$
\phi=\bigvee\{\beta \mid \beta \ll \phi\} .
$$

The condition on the right of the "|" is monotone (covariant) in $\phi$ (indeed, Example 5.12 will use the fact that it is Scott-continuous), but contravariant with respect to $\beta$ :

$$
\text { if }\left(\beta^{\prime} \leq \beta\right) \text { then }\left(\left(\beta^{\prime} \ll \phi\right) \Leftarrow(\beta \ll \phi)\right) \text {. }
$$

We want to regard the topology $\Sigma^{X}$ as another space, but the subset $\{\beta \mid \beta \ll \phi\}$ is not a sober subspace (in the Scott topology), since it isn't closed under $\bigvee$.

Remark 3.10. This means that we have, after all, to make a distinction between the exponential space $\Sigma^{X}$ and the set (albeit structured) of open subspaces of $X$. We shall write $\left|\Sigma^{X}\right|$ for the latter, since it is the set of points of the space $\Sigma^{X}$. As there are a priori no "sets" in ASD, we have to explain what special (properties of) spaces play their role in the theory. As we shall see, one of these properties is an "equality test". 
Although locale theory plays down the underlying set functor $|-|:$ Loc $\rightarrow$ Set, since it is not faithful, this functor nevertheless exists, and the subject makes intensive use of $\left|\Sigma^{X}\right|$ in particular, this being the frame corresponding to the locale $X$. The functor $|-|$ may be characterised as the right adjoint to the inclusion Set $\rightarrow$ Loc that equips any set with its discrete topology, i.e. the powerset considered as a frame. In fact, this right adjoint is precisely what we have to add to the computably motivated axioms of abstract Stone duality given in this paper, in order to make them agree with the "official" theory of locally compact locales over an elementary topos $[\mathrm{H}]$ (which writes $\Omega X$ or $U \Sigma^{X}$ for $\left|\Sigma^{X}\right|$ ). In other words, it distinguishes between the two leading models in Remark 3.1

This adjunction says that there is a map $\varepsilon:\left|\Sigma^{X}\right| \rightarrow \Sigma^{X}$ that is couniversal amongst maps $\beta: N \rightarrow \Sigma^{X}$ from the objects $N$ of a certain full subcategory, so $\beta$ factors uniquely as $N \rightarrow\left|\Sigma^{X}\right| \rightarrow \Sigma^{X}$. However, the couniversal object $\left|\Sigma^{X}\right|$ cannot exist in the computable theory: besides being uncountable, its equality test would solve the Halting Problem. So we have to develop alternatives to it. In traditional language, all we need is that any $\phi \in \Sigma^{X}$ be expressible as a directed join of $\beta^{n} \mathrm{~s}$, as in Definition 2.3.

Returning to the problem of Definition 2.1(c), we must use codes for (basic) open sets, since we cannot define $\ll$ in abstract Stone duality using the open set $\beta: \Sigma^{X}$ itself. Thus Remark 6.13 will replace the formula $\beta \ll \phi$ (with variables $\beta$ and $\phi$ ) by

$$
n: N, \phi: \Sigma^{X} \vdash\left(\beta^{n} \ll \phi\right): \Sigma,
$$

where $\beta^{n}$ is the basic open subspace with code $n$.

\section{Sets, Unions AND BASES}

Marshall Stone showed us that we should always look for the topology on mathematical objects, and ASD was called after him partly because this is its fundamental message too. However, our discussion of infinitary joins and computable bases in Definitions 1.5]and 2.1(c) has shown that this topology may still be the discrete one, i.e. that we need sets after all. Since we have no sets as such, we think of these objects as "discrete" spaces. In ASD we take this particular word to mean that there is an internal notion of equality, but the logical structure that we need to express the join is existential quantification.

Definition 4.1. An object $N \in$ obS is discrete if there is a pullback

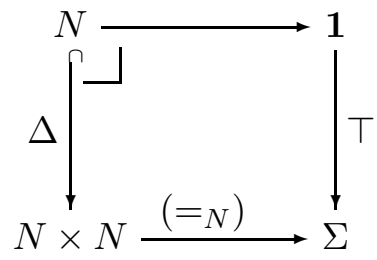

i.e. the diagonal $N \hookrightarrow N \times N$ is open. We may express this symbolically by the rule

$$
\text { for } \Gamma \vdash n, m: N, \quad \frac{\Gamma \vdash n=m: N}{\overline{\Gamma \vdash(n=N m) \Leftrightarrow \top: \Sigma}}
$$

The $(=)$ above the line denotes externally provable equality of terms of type $N$, whilst $(=N)$ below the line is the new internal structure. Categorically, $(n, m): \Gamma \rightarrow N \times N$ and $!: \Gamma \rightarrow \mathbf{1}$ provide a cone for the pullback iff $\left(n={ }_{N} m\right) \Leftrightarrow \top$, and in just this case $(n, m)$ factors through $\Delta$, i.e. $n=m$ as morphisms. 
We also write $\{n\}: \Sigma^{N}$ for the singleton predicate $\lambda m .\left(n={ }_{N} m\right)$ but $\{n\}:$ List $N$ for the singleton list.

Beware that this notion of discreteness says that the diagonal and singletons are open, but not necessarily that all subspaces of $N$ are open. For example, the Gödel numbers for non-terminating programs form a closed but not open subspace of $\mathbb{N}[\mathbb{D}, \mathbb{F}$, because the classically continuous map $\mathbb{N} \rightarrow \Sigma$ that classifies it is not computable (Definition [1.6).

Definition 4.2. Similarly, an object $H$ is Hausdorff if $H \longrightarrow H \times H$ is closed, i.e. coclassified by $\left(\neq_{H}\right): H \times H \rightarrow \Sigma$, so

$$
\text { for } \Gamma \vdash a, b: H, \quad \frac{\Gamma \vdash a=b: H}{\overline{\Gamma \vdash\left(a \neq_{H} b\right) \Leftrightarrow \perp: \Sigma}}
$$

Notice that equality of the terms $a, b: H$ corresponds to a sort of doubly negated internal equality, so this definition carries the scent of excluded middle. Like that of a closed subspace, it was chosen on the basis of topological rather than logical intuition. As we saw in Example 1.12(b) discrete spaces need not be Hausdorff.

Definition 4.3. An object $N \in$ obS is overt if $\Sigma^{!_{N}}$ (where $!_{N}$ is the unique map $N \rightarrow \mathbf{1}$ ) has a left adjoint, $\exists_{N}: \Sigma^{N} \rightarrow \Sigma$, with respect to the intrinsic order (Definition 3.7). Then

$$
\text { for } \Gamma \vdash \sigma: \Sigma, \phi: \Sigma^{N}, \quad \frac{\Gamma, x: N \vdash \phi x \Rightarrow \sigma: \Sigma}{\overline{\Gamma \vdash \exists x . \phi x \Rightarrow \sigma: \Sigma}}
$$

where we write $\exists x: N . \phi x$ or just $\exists x . \phi x$ for $\exists_{N}(\lambda x: N . \phi x)$. The Frobenius law,

$$
\sigma \wedge \exists x . \phi x \Leftrightarrow \exists x . \sigma \wedge \phi x
$$

may be derived from the Phoa principle (Axiom [3.6), and Beck-Chevalley is also automatic [C, Section 8]. The lattice dual of this definition is the subject of the next section.

Axiom 4.4. $\mathcal{S}$ has a natural numbers object $\mathbb{N}$, i.e. an object that admits primitive recursion at all types, and so is discrete and Hausdorff. We also require $\mathbb{N}$ to be overt.

In order to prove the results that we expect by induction, equational hypotheses must be allowed in the context $\Gamma$ of each judgement. See [E, §2] for a discussion of this.

Remark 4.5. Since the object $N$ over which the codes range is discrete, its intrinsic order $\leq$ is trivial [C, Lemma 6.2]. However, the structure $(N, 0,1,+, \star)$ of an abstract basis is, at least morally, supposed to be that of a distributive lattice (Remark 1.8). But this is an "imposed" structure, i.e. one that is only defined by the explicit specification of $(0,1,+, \star)$, rather than by its relationship to the other objects in the category. We are completely at liberty to consider functions that preserve, reverse or disregard the associated imposed order relation $\preccurlyeq$ that may be defined from + and $\star$ (Definition 13.10). Indeed, the need to use conditions that are contravariant with respect to the natural order was precisely the reason for distinguishing between $N$ (or $\left|\Sigma^{X}\right|$ ) and $\Sigma^{X}$ in Remark 3.9.

We typically use the letter $N$ for an overt discrete object, as its topological properties are like those of the natural numbers $(\mathbb{N})$, though the foregoing remarks do not give it either arithmetical or recursive structure. So when we write $\left(\beta^{n}\right)$ for the basis of open subspaces, we intend a family, not a sequence. The use of the letter $N$ is merely a convention, like $K$ for compact spaces; the letter $I$ (for indexing set) is often used elsewhere in the situations where we use $N$ below, but it has acquired another conventional use in abstract Stone duality 
(cf. Lemma 7.4). The notation and narrative may give the impression of countability, but bases in the classical model (Remark 3.1(a)) may be indexed by any set, however large you please. Nevertheless, I make no apology for this impression, as I consider $\aleph_{1}$ and the like to have no place in topology. I also suspect that occurrences of "sequences" and "countable sense subsets" in the subject betray the influence of objects whose significant property is overtness and not recursion.

Theorem 4.6. The full subcategory of overt discrete spaces is a pretopos, i.e. we may form products, equalisers and stable disjoint unions of them, as well as quotients by open equivalence relations [C, Section 11]. If the "underlying set" functor in Remark 3.10 exists, as in the classical models LKSp and LKLoc, then the overt discrete spaces form an elementary topos $[\mathrm{H}]$.

The combinatorial structures of most importance to us, however, are the following:

Theorem 4.7. Assuming a "linear fixed point" axiom (that any $F: \Sigma^{U} \rightarrow \Sigma^{V}$ preserves joins of ascending sequences), every overt discrete object $N$ generates a free semilattice $\mathrm{K} N$ and a free monoid List $N$ in $\mathcal{S}$, which satisfy primitive recursion and equational induction schemes, and are again overt discrete objects [E].

This result is easy to see in the two cases of primary interest, namely the classical and term models (Remark 3.1). In the classical ones (LKSp and LKLoc), overt discrete spaces are just sets with the discrete topology, and form a topos. In this case the general constructions of List $(-)$ and $\mathrm{K}(-)$ are well known: $\mathrm{K} N$ is often called the finite powerset.

Remark 4.8. In the term model, on the other hand, we shall find in Section 8 that $\mathbb{N}$ itself is adequate to index the bases of all definable objects. Moreover, any definable overt discrete space $N$ is in fact the subquotient of $\mathbb{N}$ by a some open partial equivalence relation (Corollary 9.2). This both allows us to construct $\operatorname{List}(N)$ and $\mathrm{K}(N)$, and also to extend any $N$-indexed basis to an $\mathbb{N}$-indexed one. There is, therefore, no loss of generality in taking all bases in this model to be indexed by $\mathbb{N}$, if only as a method of "bootstrapping" the theory.

To construct List $(\mathbb{N})$, we could use encodings of pairs, lists and finite sets of numbers as numbers. However, it is much neater to replace $\mathbb{N}$ with the set $\mathbb{T}$ of binary trees. Like $\mathbb{N}, \mathbb{T}$ has one constant (0) and one operation, but the latter is binary (pairing) instead of unary (successor), and the primitive recursion scheme is modified accordingly. Hence $\mathbb{T} \cong\{0\}+\mathbb{T} \times \mathbb{T} \cong \operatorname{List}(\mathbb{T})$, whereas $\mathbb{N} \cong\{0\}+\mathbb{N}$. The encoding of lists in $\mathbb{T}$ has been well known to declarative programmers since LISP: 0 denotes the empty list, and the "cons" $h:: t$ is a pair.

Membership of a list is easily defined by list recursion, as are existential and universal quantification, i.e. finite disjunction and conjunction.

$$
\begin{array}{rlrl}
(\lambda m . m \in 0) n & \equiv \perp & (\lambda m . m \in h:: t) n & \equiv(h=n) \vee(\lambda m . m \in t) n \\
\forall m \in 0 . \phi m & \equiv \top \quad \forall m \in h:: t . \phi m & \equiv \phi h \wedge \forall m \in t . \phi m \\
\exists m \in 0 . \phi m & \equiv \perp & \exists m \in h:: t . \phi m & \equiv \phi h \vee \exists m \in t . \phi m
\end{array}
$$

Notation 4.9. The notation that we actually use in this paper conceals the preceding discussion. The letter $N$ may just stand for $\mathbb{T}$ in the term model, but may denote any overt discrete space. So, in the classical models, $N$ is a set, or an object of the base topos, equipped with the discrete topology.

We use $\operatorname{Fin}(N)$ to mean $\mathrm{K}(N)$ or List $(N)$ ambiguously. Since they are respectively the free monoids on $N$ with and without the commutative and idempotent laws, this is 
legitimate so long as their interpretation also obeys these laws. But this is easy, as the interpretation is usually in $\Sigma^{X}$, with either $\wedge$ or $\vee$ for the associative operation. In fact, these two interpretations in $\Sigma^{\Sigma^{N}}$ are jointly faithful $[\mathrm{E}]$.

We are now ready to state the central assumption of this paper.

Axiom 4.10. The Scott principle: for any overt discrete object $N$,

$$
\Phi: \Sigma^{\Sigma^{N}}, \xi: \Sigma^{N} \vdash \Phi \xi \Leftrightarrow \exists \ell: \operatorname{Fin}(N) . \Phi(\lambda n . n \in \ell) \wedge \forall n \in \ell . \xi n .
$$

Notice that the Phoa principle (Axiom 3.6) is the special case with $N=\mathbf{1}$ and so $\mathrm{K}(N)=$ $\{\mathbf{0}, \mathbf{1}\}$, whilst the "linear fixed point" axiom in Theorem 4.7 can easily be shown to be equivalent to the case $N=\mathbb{N}$. More generally, the significance of this axiom is that it forces every object to have and every map to preserve directed joins (which we have yet to define), and so captures the important properties that are characteristic of topology and domain theory.

Remark 4.11. The lattice $\Sigma^{X}$ has intrinsic $M$-indexed joins, for any overt discrete object $M$. These are given by $\lambda x . \exists m: M . \phi^{m} x$, and are preserved by any $\Sigma^{f}$ [C, Corollary 8.4].

In speaking of such "infinitary" joins in $\Sigma^{X}$, we are making no additional assertion about lattice completeness: there are as many joins in each $\Sigma^{X}$ as there are overt objects, no more, no fewer. In particular, there are not "enough" to justify impredicative definitions such as the interior of a subspace, or Heyting implication (though these can be made in the context of the "underlying set" assumption in Remark $3.10[\mathrm{H}]$ ).

Moreover, we use the symbol $\exists$ to emphasise that our joins are internal to our category $\mathcal{S}$, whereas those in locale theory (written $\bigvee$ ) are external to LKLoc, involving the topos (Set) over which it is defined. This distinction will, unfortunately, become a little blurred because of the need to compare the ideas of abstract Stone duality with those of traditional topology and locale theory. This happens in particular in the definition of compactness (Definition 5.2), the way-below relation (Definition 2.1(b) and Remark 6.14) and the characterisation of finite spaces (Theorem 9.10)

Remark 4.12. When we use $M$-indexed joins, we shall need $M$ to be a dependent type, given, in traditional comprehension notation (not that of [B] $)$, by

$$
M \equiv\left\{n: N \mid \alpha_{n}\right\} \subset N,
$$

where $\alpha_{n}$ selects the subset of indices $n$ for which $\phi^{n}: \Sigma^{X}$ is to contribute to the join. In practice, this subset is always open, so $\alpha_{n}: \Sigma$. The sub- and super-script notation here (and in $[\mathrm{H}]$ ) indicates that $\phi^{n}$ typically varies covariantly and $\alpha_{n}$ contravariantly with respect to an imposed order on $N$. Indeed there would be no point in using $\alpha_{n}$ to select which of the $\phi$ s to include in the join if this had to be an upper subset, as the result would always be the greatest element, whilst it is harmless to close the subset downwards.

This means that, when using the existential quantifier, we can avoid introducing dependent types by defining

$$
\exists m:\left\{n: N \mid \alpha_{n}\right\} \cdot \phi^{m} \text { as } \exists n: N . \alpha_{n} \wedge \phi^{n} .
$$

In Section 6 we shall refer to terms like $\alpha_{n}$ of type $\Sigma$ as scalars and those like $\phi^{n}$ of type $\Sigma^{X}$ as vectors. 
Definition 4.13. A pair of families

$$
\Gamma, s: S \vdash \alpha_{s}: \Sigma, \quad \phi^{s}: \Sigma^{X}
$$

indexed by an overt discrete object $S$ is called a directed diagram, and the corresponding

$$
\exists s . \alpha_{s} \wedge \phi^{s}
$$

is called a directed join (cf. Definition 2.1(a) , if

(a) $\alpha_{s} \Leftrightarrow \top$ for some $s: S$ that we call 0 (but see Lemma 4.16), and

(b) $\alpha_{s+t} \Leftrightarrow \alpha_{s} \wedge \alpha_{t}$ and $\phi^{s+t} \geq \phi^{t} \vee \phi^{s}$ for some binary operation $+: S \times S \rightarrow S$.

In this, $\alpha_{s} \wedge \alpha_{t}$ means that both $\phi^{s}$ and $\phi^{t}$ contribute to the join, so for directedness in the informal sense, we require some $\phi^{s+t}$ to be above them both (covariance), and also to count towards the join, for which $\alpha_{s+t}$ must be true.

Although the letter $S$ stands for semilattice, in order to allow concatenation of lists to serve for + (and the empty list for 0 ), we do not require this operation to be commutative or idempotent (or even associative).

Remark 4.14. By the Scott principle, any $\Gamma \vdash F: \Sigma^{\Sigma^{X}}$ preserves directed joins, in the sense that

$$
F\left(\exists s . \alpha_{s} \wedge \phi^{s}\right) \Leftrightarrow \exists s . \alpha_{s} \wedge F \phi^{s} .
$$

Notice that $F$ is attached to the "vector" $\phi^{s}$ and not to the scalar $\alpha_{s}$, since the join being considered is really that over the subset $M \equiv\left\{s \mid \alpha_{s}\right\}$.

This is proved in Theorem 9.6, so it is one of the points on which the logical proofs lag some way behind the topological intuitions. Of course, this result could have been used in place of Axiom 4.10, but I feel that I made an important point in Tay91 by showing how sobriety (actually the slightly weaker notion of repleteness) transmits Scott continuity from the single object $\Sigma^{N}$ to the whole category.

Remark 4.15. After the separation of directed joins from finite ones $(\perp, \vee)$, the behaviour of the latter corresponds much more closely to that of meets $(T, \wedge)$. So, although Scott continuity, strictly speaking, breaks the lattice duality that we enjoyed in [C, D, we shall still often be able to treat meets and joins at the same time. We sometimes use the symbol $\odot$ for either of them, for example in Lemma 16.11. This means that we can try to transform arguments about open, discrete, overt, existential, ... things into their lattice duals about closed, Hausdorff, compact, universal, ... things. Such lattice dual results seem to be far more common that anyone brought up with intuitionistic logic or locale theory might expect. For example, there is a dual basis expansion, in which $\exists \wedge$ are replaced by $\forall \vee$, but we must leave that to later work.

Lemma 4.16. For Scott continuity, it suffices that the family be inhabited, in the sense that $\exists s: S . \alpha_{s} \Leftrightarrow \top$, and have a binary operation + with the properties stated.

In this paper, 0 will typically be the empty list, but in [I, J] we shall use $\mathbb{Q}$ with max or min, which have no semilattice unit.

Proof. $\quad$ Define $S^{\prime} \equiv S+\{0\}, \alpha_{0} \equiv \top$ and $\phi^{0} \equiv \perp$. Then

$$
\exists s: S^{\prime} . \alpha_{s} \wedge \phi^{s} \Leftrightarrow \alpha_{0} \wedge \phi^{0} \vee \exists s: S . \alpha_{s} \wedge \phi^{s} \Leftrightarrow \exists s: S . \alpha_{s} \wedge \phi^{s},
$$

but we need $\exists s: S . \alpha_{s} \Leftrightarrow \top$ for

$$
F \perp \Leftrightarrow F \perp \wedge \exists s: S . \alpha_{s} \Leftrightarrow \exists s: S . \alpha_{s} \wedge F \perp \Rightarrow \exists s: S . \alpha_{s} \wedge F \phi^{s} .
$$


Then

$$
\begin{aligned}
F\left(\exists s: S . \alpha_{s} \wedge \phi^{s}\right) & \Leftrightarrow F\left(\exists s: S^{\prime} . \alpha_{s} \wedge \phi^{s}\right) \\
& \Leftrightarrow \exists s: S^{\prime} \cdot \alpha_{s} \wedge F \phi^{s} \\
& \Leftrightarrow \alpha_{0} \wedge F \phi^{0} \vee \exists s: S . \alpha_{s} \wedge F \phi^{s} \\
& \Leftrightarrow \exists s: S \cdot \alpha_{s} \wedge F \phi^{s} .
\end{aligned}
$$$$
\text { above }
$$

Inhabitedness is all that we require of a join for it to satisfy the dual distributive or Frobenius law, $c f$. Lemma 2.7.

Lemma 4.17. If $\exists n . \alpha_{n} \Leftrightarrow \top$ then $\phi \vee\left(\exists n . \alpha_{n} \wedge \psi^{n}\right)=\exists n . \alpha_{n} \wedge\left(\phi \vee \psi^{n}\right)$.

The most dramatic effect that Scott continuity has on the presentation of general topology is the way in which it simplifies the notion of compactness, to which we now turn.

\section{Compact subspaces}

Returning from logic to topology, recall from Definition 1.5 that we need to represent points, open subobjects and compact subobjects in our calculus. These will be given by terms of type $X, \Sigma^{X}$ and $\Sigma^{\Sigma^{X}}$ respectively, but whilst the first two correspond exactly, the question of which second order terms denote compact subspaces is more complicated.

We pick up the treatment of compact objects from [C, Sections 7-8], and show how this leads to the representation of compact subobjects as quantifiers or modal operators. Following [HM81], these should preserve finite meets and directed joins, but the basic idea of ASD is that the directed joins come for free. A compact subobject is therefore represented by a term $A: \Sigma^{\Sigma^{X}}$ for which $A \top \Leftrightarrow \top$ and $A(\phi \wedge \psi) \Leftrightarrow A \phi \wedge A \psi$.

This abstract idea works very well as far as the relationship between compact and closed subspaces of a compact Hausdorff space. But then we run into some trouble, because at that point we actually need to use Scott continuity, but our logical structure will not be strong enough to do this until Section [9. There is an alternative treatment in [J] that avoids this problem by restricting to the Hausdorff case, and in particular $\mathbb{R}$ and $\mathbb{R}^{\mathbf{n}}$; you may prefer to read that instead of this section, or in parallel with it.

In the experimental spirit, we try to go further down the path laid by [HM81, JS966, but we stumble into more and more potholes, showing how much more work needs to be done in ASD to make it into a full theory of general topology. But Jung and Sünderhauf do have something interesting to show us when we make this excursion, namely a very close duality between compact and open subobjects of stably locally compact objects.

The abstract analogue of the way in which locale theory treats local compactness is much simpler than this. In that case any term $A: \Sigma^{\Sigma^{X}}$ may be used - it doesn't have to preserve meets.

Remark 5.1. Traditionally ${ }^{2}$, a topological space $K$ has been defined to be compact if every open cover, i.e. family $\left\{U_{s} \mid s \in S\right\}$ of open subspaces such that $K=\bigcup_{s} U_{s}$, has a finite subcover, $F \subset S$ with $K=\bigcup_{s \in F} U_{s}$. If the family is directed (Definition 2.1(a) then $F$ need only be a singleton, i.e. there is already some $s \in S$ with $K=U_{s}$.

The Scott topology on the lattice $\left|\Sigma^{K}\right|$ offers a simpler way of saying that $K$ is compact. In this lattice, $T$ denotes the whole of $K$, so compactness says that if we can get into the

\footnotetext{
${ }^{2}$ In fact Bourbaki Bou66 I 9.3] relegated this formulation to Axiom $\mathrm{C}^{\prime \prime}$, also calling it "the axiom of Borel-Lebesgue". The older intuitions from analysis involve the existence of cluster points of sequences or nets of points, or of filters of subsets.
} 
subset $\{\top\} \subset\left|\Sigma^{K}\right|$ by a directed join $\bigcup_{s} U_{s}$ then some member $U_{s}$ of the family was already there $^{3}$. In other words, $\{\top\} \subset \Sigma^{K}$ is an open subset in the Scott topology on the lattice.

Definition 5.2. In abstract Stone duality we say that an object $K$ is compact if there is a pullback

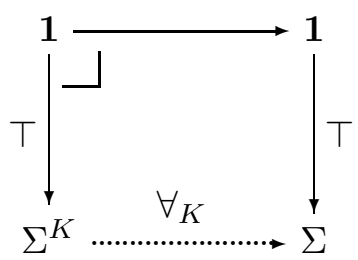

Using the fact that $\Sigma$ classifies open subobjects (Remark [3.2), together with its finitary lattice structure (but not the Scott principle), [C, Proposition 7.10] shows that $\forall_{K}$ exists with this property iff it is right adjoint to $\Sigma^{!_{K}}$ (where $!_{K}$ is the unique map $K \rightarrow \mathbf{1}$ ). It is then demonstrated that this map does indeed behave like a universal quantifier in logic (the corresponding existential quantifier was given by Definition 4.3).

Lemma 5.3. If $K$ and $L$ are compact objects then $K+L$ is also compact, as is $\emptyset$.

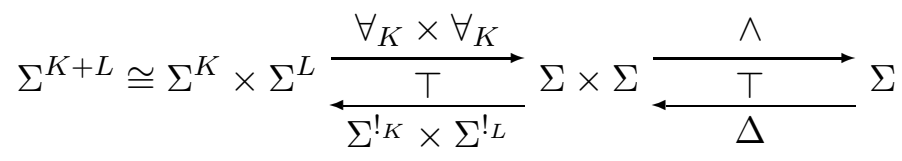

Proof. $\quad \forall_{K+L}(\phi, \psi) \Leftrightarrow \forall_{K} \phi \wedge \forall_{L} \psi$ and $\forall_{\emptyset}=\top: \Sigma^{\emptyset} \cong \mathbf{1} \rightarrow \Sigma$.

The following result is the well known fact that the direct image of any compact subobject is compact (whereas inverse images of open subobjects are open).

Lemma 5.4. Let $K$ be a compact object and $p: K \rightarrow X$ be $\Sigma$-epi $\boldsymbol{i}^{4}$, i.e. a map for which $\Sigma^{p}: \Sigma^{X} \rightarrow \Sigma^{K}$ is mono. Then $X$ is also compact, with quantifier $\forall_{X}=\forall_{X} \cdot \Sigma^{p}$.

Proof. The given quantifier, $\Sigma^{!} \mathfrak{H}_{K} \dashv \forall_{K}$, satisfies the inequalities

$$
\begin{gathered}
\text { id } \leq \forall_{K} \cdot \Sigma^{p} \cdot \Sigma^{!_{X}}=\forall_{K} \cdot \Sigma^{!_{K}} \\
\Sigma^{p} \cdot \Sigma^{!_{X}} \cdot \forall_{K} \cdot \Sigma^{p}=\Sigma^{!_{K}} \cdot \forall_{K} \cdot \Sigma^{p} \leq \Sigma^{p},
\end{gathered}
$$

from which we deduce $\Sigma^{!} X \dashv \forall_{K} \cdot \Sigma^{p}$, since $\Sigma^{p}$ is mono.

What becomes of the quantifier $\forall_{K}$ when $K$ no longer stands alone but is a subobject of some other object $X$ ? We see that any compact subobject $K \subset X$ defines a map $A: \Sigma^{X} \rightarrow \Sigma$ or $A: \Sigma^{\Sigma^{X}}$ that preserves $\top$ and $\wedge$. We shall call this a necessity $(\square)$ modal operator, rather than a quantifier, since we have lost the instantiation or $\forall$-elimination rule $A \phi \Rightarrow \phi x$.

Lemma 5.5. Let $i: K \rightarrow X$ be any map, with $K$ compact. Then $A \equiv \forall_{K} \cdot \Sigma^{i}: \Sigma^{X} \rightarrow \Sigma$ preserves $\top$ and $\wedge$. Moreover $A \phi \equiv \forall_{K}\left(\Sigma^{i} \phi\right) \Leftrightarrow \top$ iff $\Sigma^{i} \phi=\top_{K}$.

Proof. $\quad \Sigma^{i}$ preserves the lattice operations and $\forall_{K}$ is a right adjoint.

\footnotetext{
${ }^{3}$ This is therefore an externally defined join, cf. Remark 4.11

${ }^{4}$ In our category, where every object is a subobject of some $\Sigma^{Y}, \Sigma$-epi is the same as epi. The name has been inherited from synthetic domain theory, a model of which is a topos, only some of whose objects (namely the predomains) may be so embedded.
} 
Remark 5.6. In traditional language, for any open set $U \subset X$ classified by $\phi: \Sigma^{X}$, the predicate $A \phi$ says whether $K \subset U$. Just as $\forall_{K}: \Sigma^{K} \rightarrow \Sigma$ classifies $\{\top\} \subset \Sigma^{K}$, the map $A: \Sigma^{X} \rightarrow \Sigma$ classifies a Scott-open family $\mathcal{F}$ of open subspaces of $X$, namely the family of neighbourhoods of $K$. Preservation of $\wedge$ and $\top$ says that this family is a filter.

Notice that we have an example of the alternation $K \subset U$ of compact and open subobjects that we saw in Lemma 1.2. Also, recall from Definition 1.5 that we wanted to test $x \in U$ and $K \subset U$; these are expressed by the $\lambda$-applications $\phi x$ and $A \phi$ respectively.

Lemma 5.7. (a) If $K=\emptyset$ then $A=\lambda \phi$. $\top$, so in particular $A \perp \Leftrightarrow \top$.

(b) If $K=\{p\}$ then $A=\lambda \phi . \phi p \equiv \eta_{X}(p)$, which is prime (Axiom 3.3(b) and preserves all four lattice operations, in particular $A \perp \Leftrightarrow \perp$.

(c) If $K \cup L$ exists then $A_{K \cup L} \phi \Leftrightarrow A_{K} \phi \wedge A_{L} \phi$.

(d) If $K \subset L$ then $A_{K} \geq A_{L}$.

(e) Even when $K \cap L$ is compact, $A_{K} \vee A_{L}$ need not be its modal operator.

Proof.

(a) $\Sigma^{K}=\mathbf{1}$ and $\forall_{K}=\lambda \psi$. $\top$, so $A \equiv \forall_{K} \cdot \Sigma^{i}=\lambda \phi$. $\top$.

(b) $\Sigma^{K}=\Sigma, \forall_{K}=$ id and $\Sigma^{i}=\lambda \phi . \phi p$.

(c) Since $K+L \longrightarrow K \cup L \longrightarrow X$, we have $A_{K \cup L}=A_{K+L}=A_{K} \wedge A_{L}$ by Lemmas 5.3 and 5.4

(d) This follows from the previous part, since $K \cup L=L$.

(e) Classically, of course, $K \cap L \subset U$ does not imply $(K \subset U \vee L \subset U)$. For example, let $K=\{p\}$ and $L=\{q\}$, where $p, q \in X$ are incomparable points in the specialisation order (Definition [3.7), so $K \cap L=\emptyset$. Then $A_{K \cap L} \perp \Leftrightarrow \top$ but $\perp \Leftrightarrow A_{K} \perp \vee A_{L} \perp$.

Corollary 5.8. $A_{K} \perp \Leftrightarrow \top$ if $K$ is empty, $\perp$ if it's inhabited.

Remark 5.9. Classically, any compact (sub)space that satisfies $A_{K} \perp \Leftrightarrow \perp$ must therefore contain a point, by excluded middle, but Choice is still needed to find it Joh82, Exercise VII 4.3]. In the localic formulation, it is a typical use of the maximality principle to find a prime filter containing $\mathcal{F}$, so $A \leq P$ in our notation.

The situation in which open and compact subobjects interact extremely well is that of a compact Hausdorff object (Definitions 4.2 and [5.2).

Lemma 5.10. Let $X$ be a compact Hausdorff object and $\phi: \Sigma^{X}$. Then

$$
\begin{array}{ll}
(x \neq y) \vee \phi x & \Leftrightarrow(x \neq y) \vee \phi y \\
\phi x & \Leftrightarrow \forall y .(x \neq y) \vee \phi y \\
(x \neq x) & \Leftrightarrow \perp \\
(x \neq y) & \Leftrightarrow(y \neq x) \\
(x \neq z) & \Rightarrow(x \neq y) \vee(y \neq z)
\end{array}
$$

Proof. These are the lattice duals of $(x=y) \wedge \phi x \Leftrightarrow(x=y) \wedge \phi y, \phi x \Leftrightarrow \exists y .(x=y) \wedge \phi y$, reflexivity, symmetry and transitivity in any overt discrete object, and the following proof is just the dual of that in [C, Lemma 6.7]. 
The closed subobject $\Delta: X \subset X \times X$ is co-classified by $\left(\neq_{X}\right)$, so the corresponding nucleus, in the senses of both locale theory $\left(j \equiv \Delta_{*} \cdot \Delta^{*}\right)$ and of abstract Stone duality $\left(E \equiv \forall_{\Delta} \cdot \Sigma^{\Delta}\right.$, Axiom 3.3(c) and Lemma 3.8) takes

$$
\phi: \Sigma^{X \times X} \quad \text { to } \quad \lambda x y .(x \neq y) \vee \phi y .
$$

Consider in particular $\psi \equiv \Sigma^{p_{0}} \phi$, or $\psi(x, y) \equiv \phi x$; then

$$
\forall_{\Delta} \phi=\forall_{\Delta} \cdot \Sigma^{\Delta} \cdot \Sigma^{p_{0}} \phi=\forall_{\Delta} \cdot \Sigma^{\Delta} \psi=\left(\forall_{\Delta} \perp\right) \vee \Sigma^{p_{0}} \phi .
$$

The same thing in $\lambda$-calculus notation, and its analogue for $p_{1}$, are

$$
\left(\forall_{\Delta} \phi\right)(x, y) \Leftrightarrow(x \neq y) \vee \phi x \Leftrightarrow(x \neq y) \vee \phi y .
$$

Now apply $\forall_{p_{0}} \equiv \forall y$, so $\phi=\forall_{p_{0}} \cdot \forall_{\Delta} \phi=\forall_{p_{0}}\left(\forall_{\Delta} \perp \vee \Sigma^{p_{0}} \phi\right)$, i.e. $\phi x \Leftrightarrow \forall y .(x \neq y) \vee \phi y$. Inequality is irreflexive by definition. We deduce symmetry by putting $\phi \equiv \lambda u$. $(y \neq u)$ and the dual of the transitive law with $\phi \equiv \lambda u$. $(u \neq z)$.

The idea of the following construction is that $K \subset U$ iff $U \cup V=X$, and conversely $x \in V$ iff $K \subset X \backslash\{x\}$, where $K$ is compact and $V$ is its complementary open subobject, encoded by $A$ and $\psi$ respectively. The lattice dual of this result - that open and overt subobjects coincide in an overt discrete object — was proved for $\mathbb{N}$ in [A], Section 10].

Proposition 5.11. In any compact Hausdorff object $K$, there is a retraction $\Sigma^{K} \triangleleft \Sigma^{\Sigma^{K}}$ given by

$$
\begin{aligned}
& \psi \\
& A \quad \lambda \phi . \forall x . \psi x \vee \phi x \\
& A \quad \lambda x . A(\lambda y . x \neq y),
\end{aligned}
$$

where, if $A$ is so defined from $\psi$ then it preserves $\top$ and $\wedge$.

Later we shall show that closed and compact subobjects agree exactly.

Proof. $\quad \psi \mapsto A \mapsto \lambda x . A(\lambda y . \psi y \vee x \neq y)=\psi$, by the second part of the Lemma. Then $A \top \Leftrightarrow \top$ easily, whilst $A\left(\phi_{1} \wedge \phi_{2}\right) \Leftrightarrow A \phi_{1} \wedge A \phi_{2}$ by distributivity.

On the other hand, $A \mapsto \psi \mapsto \lambda \phi . \forall x . A(\lambda y . \phi y \vee x \neq y)$, whilst the first part of the Lemma says that $A=\lambda \phi . A(\lambda y . \forall x . \phi y \vee x \neq y)$, so for the bijection between closed and compact objects we need $A$ to commute with $\forall x$. To show this, we need to know about the Tychonov product topology on $X \times X$ (Remark 8.3), and to use Scott continuity (Theorem 9.6).

As we said, it is not obligatory that the $A$ s that we shall use to define locally compact objects in the next section actually satisfy these conditions, i.e. preserve $T$ and $\wedge$. Consider the localic situation.

Example 5.12. For $\beta \in L$ in a continuous lattice (Definition 2.1(c), the subset

$$
\uparrow \beta \equiv\{\phi \mid \beta \ll \phi\} \subset L
$$

is Scott-open, and therefore classified by some $A: \Sigma^{L}$ (Remark 3.2). However, $\uparrow \beta$ need not be a filter ( $c f$. Definition [2.9), so $A$ need not preserve $\top$ or $\wedge$. 
We obtain similar behaviour even in traditional topology. The following may seem a strange thing to do, but it will fall into place as we start to use the $\lambda$-calculus.

Notation 5.13. In A we found it useful to regard any map $F: \Sigma^{X} \rightarrow \Sigma^{Y}$ (not necessarily a homomorphism for the monad or of frames, but nevertheless Scott-continuous) as a "second class" map $\widehat{F}: Y \rightarrow X$, and to write $\mathrm{HS}$ for the category composed of such maps. The work cited there explains how they interpret "control effects" such as jumps in programming languages. We shall in particular meet $I: \Sigma^{X} \rightarrow \Sigma^{Y}$ such that $\Sigma^{i} \cdot I=$ id, where $i$ : $X \longrightarrow Y$.

Remark 5.14. Just as in Lemma 5.5] we formed the modal operator corresponding to the direct image $i K$ of $K$ along $i: K \rightarrow X$ as the composite $A=\forall_{K} \cdot \Sigma^{i}$, so we may form the direct image $\widehat{F} A$ along a second class map $\widehat{F}: K \longrightarrow X$ as $\forall_{K} \cdot F$. Its open neighbourhoods are given, as in Remark [5.6] by

$$
(\widehat{F} K \subset \phi) \Leftrightarrow\left(\forall_{K} \cdot F\right) \phi \Leftrightarrow A(F \phi) \Leftrightarrow(K \subset F \phi) .
$$

However, $\forall_{K} \cdot F$ need only be a filter when $F$ preserves $\top$ and $\wedge$.

Even when $A$ does preserve meets, and so classifies a Scott-open filter of open subspaces, it need not correspond to a (locally) compact subspace. (It does in a compact Hausdorff object, but even there we do not yet have to tools to prove it, $c f$. Proposition [5.11) We make a brief excursion into classical topology to illustrate the duality of open and compact subspaces, and their alternating inclusions (Notation 1.3). Recall from Definition 1.1 that any open subspace is the union of the compact subspaces inside it: the first result answers the dual question of when a compact subspace is the intersection of the open subspaces that contain it.

Lemma 5.15. Using excluding middle in classical topology [HM81, p221 def 2.1],

$$
\{y \in X \mid \exists x \in K . x \leq y\}=\bigcap\{U \subset X \text { open } \mid K \subset U\},
$$

where $\leq$ is the specialisation order (Definition [3.7). We call this the saturation of $K$. Hence compact subspaces of $X$ can be recovered from their modal operators iff $X$ is $\mathrm{T}_{1}$ (when $\leq$ is trivial). For a specific non-example, let $X \equiv \Sigma$ and $K \equiv\{\perp\}$, so $A \equiv \lambda \phi . \phi \perp$ and the saturation of $K$ is $\Sigma$.

What we might hope to recover from the modal operator $A$, therefore, is the saturation of $K$, as Karl Hofmann and Michael Mislove did for sober spaces [HM81, Theorem 2.16], and Peter Johnstone did for locales [Joh85. This result shows that we have identified enough of the properties of modal operators, at least in the classical model.

Lemma 5.16. Let $\mathcal{F} \subset\left|\Sigma^{X}\right|$ be a Scott-open filter of open subspaces of a sober (but not necessarily locally compact) space. Then, assuming the axiom of choice, $K \equiv \bigcap \mathcal{F} \subset X$ is a compact subspace, and $\mathcal{F}=\{U \mid K \subset U\}$.

Lemma 5.17. HM81, p221 thm 2.16] Let $K \equiv \bigcap_{s} K_{s}$ be a co-directed intersection of compact saturated subspaces of a sober space. Then $K$ is also compact saturated, and $A_{K}=$ $\bigvee A_{K_{s}}$. If all of the $K_{s}$ are inhabited then so is $K$. 
Theorem 5.18. Kel55, Problem $5 \mathrm{~F}(\mathrm{a})$ ] Compact saturated subspaces of a locally compact sober space form a continuous preframe under reverse inclusion. That is, for any compact saturated subspace $K \subset X$,

$$
K=\bigcap\{L \text { compact saturated } \mid L \prec K\} .
$$

Here $L \nVdash K$ means that there is an open subspace $U$ with $K \subset U \subset L$ ( sic - Notation 1.3), but this is equivalent to $L \ll K$, the order-reversed analogue of Definition 2.1(b), i.e.

$$
K \supset \bigcap_{s} M_{s} \Rightarrow \exists s . K \supset M_{s} .
$$

Corollary 5.19. Using Choice in classical topology, stably locally compact sober spaces enjoy the dual Wilker property (cf. Lemma 1.10): if $K \cap L \subset U$ then there are open subspaces $V$ and $W$ and compact ones $K^{\prime}$ and $L^{\prime}$ such that $K^{\prime} \cap L^{\prime} \subset U, K \subset V \subset K^{\prime}$ and $L \subset W \subset L^{\prime}$ [JS96, Theorem 23].

Proof. Use Lemma 2.7 in the continuous lattice of compact saturated subspaces under reverse inclusion. It is significant in this that the abstract joins in the lattice be given by intersections of subspaces.

Remark 5.20. There is still a problem. Even when $X$ itself is locally compact, and we have a filter $\mathcal{F}$ that is Scott-open and therefore classified by a term $A$ of type $\Sigma^{\Sigma^{X}}$, the compact subspace $K$ that they define need not be locally compact. Therefore they need not be definable as actual objects in the theory that we describe in this paper. However, an extension to and beyond the category of all locales is envisaged for later work. Preliminary investigations in this suggest that any $A: \Sigma^{\Sigma^{X}}$ that preserves $\top$ and $\wedge$ can be expressed as the universal quantifier of a compact subobject.

However, as we said at the beginning of this section, the "dual bases" that we shall introduce in the next section use the $A \mathrm{~s}$ and not the $K \mathrm{~s}$. It is a desirable property of $A$ that it be a filter, i.e. preserve $T$ and $\wedge$, because then we can fully exploit the intuitions of traditional topology. But it is not necessary: locale theory gives rise to bases that don't have this property, and both ways of doing things have their advantages.

\section{EFFECTIVE BASES}

This section introduces the first of our abstract characterisations of local compactness ((d) in the Introduction) and explores its relationship to the traditional definitions in pointset topology and locale theory.

Remark 6.1. Recall that we call a system $\left(U^{n}\right)$ of vectors in a vector space a basis if any other vector $V$ can be expressed as a sum of scalar multiples of basic vectors. Likewise, we say that a system $\left(U^{n}\right)$ of open subspaces of a topological space is a basis if any other open set $V$ can be expressed as a "sum" (union or disjunction) of basic opens.

How do we find out which basis elements contribute to the sum, and (in the case of vector spaces) by what scalar multiple? By applying the dual basis $A_{n}$ to the given element $V$, giving $A_{n} \cdot V$. Then

$$
V=\sum_{n} A_{n} \cdot V * U^{n}
$$


where

(a) $\sum$ denotes linear sum, union, disjunction or existential quantification;

(b) "scalars" in the case of topology range over the Sierpiński space;

(c) the dot denotes

- inner product of a dual vector with a vector to yield a scalar,

- that $V$ is an element of the family classified by $A_{n}$, or

- $\lambda$-application; and

(d) ' $*$ ' denotes multiplication by a scalar of a vector, or conjunction.

In abstract Stone duality, since the application of $A_{n}$ to a predicate $V: \Sigma^{X}$ yields a scalar, it must have type $\Sigma^{\Sigma^{X}}$. In the previous section we saw that (some) such terms play the role of compact subobjects.

Definition 6.2. An effective basis for an object $X$ is a pair of families

$$
n: N \vdash \beta^{n}: \Sigma^{X} \quad n: N \vdash A_{n}: \Sigma^{\Sigma^{X}},
$$

where $N$ is an overt discrete object (Section 4 ), such that every "vector" $\phi: \Sigma^{X}$ has a basis decomposition,

$$
\phi=\exists n . A_{n} \phi \wedge \beta^{n} .
$$

This is a join $\bigvee\left\{\beta^{n} \mid \alpha_{n}\right\}$ over a subset, with $\alpha_{n} \equiv A_{n} \phi$, in the sense of Remark 4.12 In more traditional topological notation, this equation says that

$$
\text { for all } V \subset X \text { open, } \quad V=\bigcup\left\{U^{n} \mid V \in \mathcal{F}_{n}\right\},
$$

where $\beta^{n}, \phi: \Sigma^{X}$ and $A_{n}: \Sigma^{\Sigma^{X}}$ classify open $U^{n}, V \subset X$ and $\mathcal{F}_{n} \subset \Sigma^{X}$ in the sense of Remark 3.2

We call $\left(\beta^{n}\right)$ the basis and $\left(A_{n}\right)$ the dual basis. The reason for saying that the basis is "effective" is that it is accompanied by a dual basis, so that the coefficients are given effectively by the above formula - it is not the computational aspect that we mean to stress at this point. The sub- and superscripts indicate the co- and contravariant behaviour of compact and open subobjects respectively with respect to continuous maps.

Remark 6.3. We shall find in Section 8 that every object that is definable in the ASD calculus (as we set it out in Sections 34) has an effective basis. However, there are plans to extend this calculus to and beyond general locales, where those results will no longer apply. Then we shall define a locally compact object to be one that has an effective basis in the above sense.

The first observation that we make about this definition expresses the inclusion $U^{n} \subset$ $K^{n}(c f$. Definition 1.5(f) . After that we see some suggestion of the role of compact subobjects, although Lemma 6.5 is too specific to be of much use, unless $X$ happens to have a basis of compact open subobjects, i.e. it is a coherent object [Joh82, Section II 3].

Lemma 6.4. If $\Gamma \vdash \phi: \Sigma^{X}$ satisfies $\Gamma \vdash A_{n} \phi \Leftrightarrow \top$ then $\Gamma \vdash \beta^{n} \leq \phi$.

Proof. Since $A_{n} \phi \Leftrightarrow \top$, the basis decomposition for $\phi$ includes $\beta^{n}$ as a disjunct. 
Lemma 6.5. If $\vdash A_{n} \beta^{n} \Leftrightarrow \top$ then $\beta^{n}$ classifies a compact open subobject.

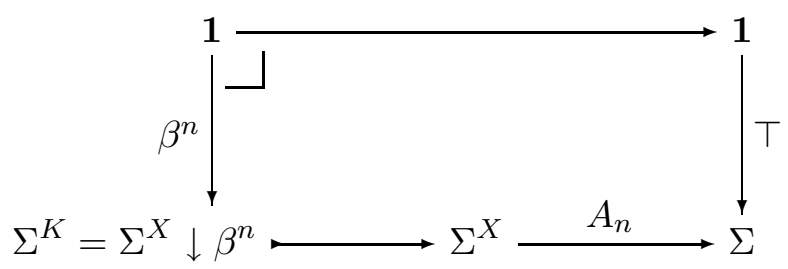

Proof. The equation $A_{n} \beta^{n} \Leftrightarrow \top$ says that the square commutes. Any test map $\phi$ : $\Gamma \rightarrow \Sigma^{X} \downarrow \beta^{n}$ that (together with ! : $\Gamma \rightarrow \mathbf{1}$ ) also makes a square commute must satisfy $\Gamma \vdash A_{n} \phi \Leftrightarrow \top$ and $\Gamma \vdash \phi \leq \beta^{n}$, but then $\phi=\beta^{n}$ by the previous result. Hence the square is a pullback, whilst $\beta^{n}=\top_{\Sigma^{K}}$, so the lower composite is $\forall_{K}$, making $K$ compact.

The following jargon will be useful:

Definition 6.6. An effective basis $\left(\beta^{n}, A_{n}\right)$ for an object $X$ is called (a) a directed or $\vee$-basis if there is some element (that we call $0 \in N$ ) such that

$$
\beta^{0}=\lambda x . \perp \quad \text { and } \quad A_{0}=\lambda \phi . \top
$$

(though $A_{0}=\top$ entails $\beta^{0}=\perp$ by Lemma 6.4) and a binary operation $+: N \times N \rightarrow N$ such that

$$
\beta^{n+m}=\beta^{n} \vee \beta^{m} \quad \text { and } \quad A_{n+m}=A_{n} \wedge A_{m} ;
$$

this definition is designed to work with Definition 4.13 it is used first in Lemma 9.8 and then extensively in Sections 11, 16,

(b) an intersection or $\wedge$-basis if $\beta^{1}=\lambda x$. $\top$ for some element (that we call $1 \in N$ ), and there is a binary operation $\star$ such that

$$
\beta^{n \star m}=\beta^{n} \wedge \beta^{m} \quad A_{n} \leq A_{n \star m} \text { and } A_{m} \leq A_{n \star m}
$$

so the intersection of finitely many basic opens is basic; this is a positive way of saying that we do have a basis instead of what is traditionally known as a $\boldsymbol{s u b}$-basis;

(c) a lattice basis if it is both $\wedge$ and $\vee$;

(d) a filter basis if each $A_{n}$ preserves $\wedge$ and $\mathrm{T}$, and so corresponds in classical topology to a compact saturated (though not necessarily locally compact) subspace $K^{n}$, by Lemma 5.16 .

(e) a prime basis if each $A_{n}$ of the form $A_{n} \phi \Leftrightarrow \phi p^{n}$ for some $p^{n}: X$ (cf. Axiom 3.3(b)], the corresponding compact subobject being $K^{n}=\left\{p^{n}\right\}$.

Any effective basis can be "up-graded" to a lattice basis by formally adding unions and intersections (Lemma 8.4 f). It can be made into a filter $\vee$-basis instead (Remark [17.6). Some of the other terminology is only applicable in special situations: if $A_{1} \top \Leftrightarrow \top$ then the object is compact, with $\forall_{X}=A_{1}$, whilst a sober topological space has a prime basis iff it is a continuous dcpo with the Scott topology (Theorem 12.11). Even when the intersection of two compact subobjects is compact, there is nothing to make $A_{n \star m}$ correspond to it, but we shall rectify this in Proposition 12.14.

Examples 6.7. Let $N$ be an overt discrete object. Then

(a) $N$ has an $N$-indexed prime basis given by

$$
\beta^{n} \equiv\{n\} \equiv \lambda x .\left(x=_{N} n\right) \text { and } A_{n} \equiv \eta_{N}(n) \equiv \lambda \phi . \phi n,
$$


because $\exists n . A_{n} \phi \wedge \beta^{n} x \equiv \exists n . \eta n \phi \wedge\{n\} x \Leftrightarrow \exists n . \phi n \wedge(x=n) \Leftrightarrow \phi x$; and (b) $\Sigma^{N}$ has a Fin $(N)$-indexed prime $\wedge$-basis given by

$$
B^{\ell} \equiv \lambda \xi . \forall m \in \ell . \xi m \quad \text { and } \quad \mathcal{A}_{\ell} \equiv \lambda \Phi . \Phi(\lambda m . m \in \ell),
$$

because $\Phi \xi \Leftrightarrow \exists \ell . \Phi(\lambda m . m \in \ell) \wedge \forall m \in \ell . \xi m$ by Axiom 4.10] (The convention that superscripts indicate covariance (Remarks 3.9] and 4.12) means that the imposed order on $\operatorname{Fin}(N)$ here is reverse inclusion of lists.)

We devote the remainder of this section to showing that every locally compact sober space or locale has an effective basis in our sense. We start with traditional topology.

Proposition 6.8. Any locally compact sober space has a filter basis.

Proof. Definitions 1.1 and 1.5(f) provide families $\left(K^{n}\right)$ and $\left(U^{n}\right)$ of compact and open subspaces such that

$$
\text { for each open } V \subset X, \quad V=\bigcup\left\{U^{n} \mid K^{n} \subset V\right\} .
$$

As the subspace $K^{n}$ is compact, Remark [5.6] defines $A_{n}: \Sigma^{X} \rightarrow \Sigma$ such that $K^{n} \subset V$ iff $A_{n} \phi \Leftrightarrow \top$, where $\beta^{n}$ and $\phi: \Sigma^{X}$ classify $U^{n}$ and $V \subset X$, so $\phi=\exists n . A_{n} \phi \wedge \beta^{n}$.

Notice how the basis decomposition "short changes" us, for individual basis elements: we "pay" $K^{n} \subset V$ but only receive $U^{n} \subset K^{n}$ as a contribution to the union. Nevertheless, the interpolation property (Lemma 1.2) ensures that we get our money back in the end.

In many examples, $U^{n}$ may be chosen to be interior of $K^{n}$, and $K^{n}$ the closure of $U^{n}$. However, this may not be possible if we require an $\vee$-basis. For example, such a basis for $\mathbb{R}$ would have as one of its members a pair of touching intervals, $(0,1) \cup(1,2)$, which is not the interior of $[0,1] \cup[1,2]$.

Remark 6.9. It is difficult to identify a substantive Theorem by way of a converse to this in traditional topology, since the $\lambda$-calculus can only by interpreted in topological spaces if they are already locally compact, and therefore have effective bases. Nevertheless, we can show that a filter basis $\left(\beta^{n}, A_{n}\right)$ can only arise in the way that we have just described.

By Lemma 5.16, each $A_{n}$ corresponds to some compact saturated subspace $K^{n}$, where

$$
\left(K^{n} \subset V\right) \Longleftrightarrow A_{n} \phi \Longrightarrow\left(U^{n} \subset V\right),
$$

$\beta^{n}$ and $\phi$ being the classifiers for $U^{n}$ and $V$ as usual. Since $K^{n}=\bigcap\left\{V \mid K^{n} \subset V\right\}$, we must have $U^{n} \subset K^{n}$. Then, given $x \in V$, so $\phi x \Leftrightarrow \top$, the basis decomposition $\phi x \Leftrightarrow$ $\exists n$. $A_{n} \phi \wedge \beta^{n} x$, means that $x \in U^{n} \subset K^{n} \subset V$, as in Definition 1.1.

Example 6.10. The closed real unit interval has a filter $\wedge$-basis with

$$
\begin{aligned}
& \beta^{q \pm \epsilon} \equiv(q \pm \epsilon) \equiv \lambda x \cdot|x-q|<\epsilon \\
& A_{q \pm \delta} \equiv[q \pm \delta] \equiv \lambda \phi . \forall x \cdot|x-q| \leq \delta \Rightarrow \phi x
\end{aligned}
$$

where $\epsilon, \delta>0$ and $q$ are rational, and we re-deploy the interval notation of Example 1.4 in our $\lambda$-calculus. We also write $\langle q \pm \delta\rangle$ for a variable that ranges over the codes, as opposed to the open $(q \pm \delta)$ and compact $[q \pm \delta]$ intervals that it names. The imposed order is given by comparison of the radii $\epsilon$ or $\delta$. 
Proof. Let $x \in U \subset[0,1]$; then, for some $\epsilon>0, \forall y .|x-y|<\epsilon \Rightarrow y \in U$. So with $\delta \equiv \frac{1}{2} \epsilon$, and $q$ rational such that $|x-q|<\delta$,

$$
\forall y .|y-q| \leq \delta \Rightarrow y \in U \quad \wedge \quad x \in\{y|| y-q \mid<\delta\}
$$

which is $\exists\langle q \pm \delta\rangle . A_{q \pm \delta} \phi \wedge \beta^{q \pm \delta} x$ in our notation.

Example 6.11. Recall that any compact Hausdorff space $X$ has a stronger property called regularity: if $C \subset X$ is closed with $x \notin C$ then there are $x \in U \pitchfork V \supset C$ with $U, V \subset X$ open and disjoint. Writing $K=X \backslash V$ and $W=X \backslash C$ for the complementary compact and open subspaces, this says that given $x \in W$, we can find $x \in U \subset K \subset W$, as in Definition 1.1. Let $\left(\beta^{n}, \gamma_{n}\right)$ classify a sufficient computable family $\left(U^{n} \not V_{n}\right)$ of disjoint open pairs, and put $A_{n} \equiv \lambda \phi . \forall x . \phi x \vee \gamma_{n} x$, which corresponds to the compact complement of $V_{n}$ (Proposition 5.11). Then $\left(\beta^{n}, A_{n}\right)$ is a filter basis for $X$. It is a lattice basis if the families $\left(\beta^{n}\right)$ and $\left(\gamma_{n}\right)$ are sublattices, with $\gamma_{0}=\top, \gamma_{1}=\perp, \gamma_{n+m}=\gamma_{n} \wedge \gamma_{m}$ and $\gamma_{n \star m}=\gamma_{n} \vee \gamma_{m}$.

Remark 6.12. In these idioms of topology, where we say that there "exists" an open or compact subobject within certain bounds, that subobject may typically be chosen to be basic, and the existential quantifier in the assertion ranges over the set (overt discrete object) $N$ of indices for the basis, rather than over the topology $\Sigma^{X}$ itself. For example, this is the case with the Wilker property in Lemmas 1.10 and 2.7

Now we turn to locale theory.

Proposition 6.13. Any locally compact locale has a lattice basis.

Proof. The localic definition is that the frame $L$ be a distributive continuous lattice (Definition 2.1(c), so

$$
\text { for all } \phi \in L, \quad \phi=\bigvee\{\beta \in L \mid \beta \ll \phi\},
$$

and by Example 5.12, $\uparrow \beta \equiv\{\phi \mid \beta \ll \phi\}$ is classified by some $A: \Sigma^{L}$.

This means that there is a basis decomposition

$$
\phi=\exists n \cdot \beta^{n} \wedge A_{n} \phi, \quad \text { where } \quad A_{n} \equiv \lambda \phi .\left(\beta^{n} \ll \phi\right),
$$

so $\left(\beta^{n}, A_{n}\right)$ is a lattice basis.

Recall, however, from Remark 3.9 that we must consider this basis to be indexed by the underlying set, $|L|$, of the frame $L$. Thus $N \equiv|L|$, whilst $\beta^{(-)}: N \rightarrow L$ is the couniversal map from an overt discrete object $N$ to $L$.

In fact it is enough for the image of $N$ to generate $L$ under directed joins (Definition 2.3). There is no need for $N$ to be the couniversal way of doing this, and so no need for the underlying set functor $|-|$.

Remark 6.14. The first part of the converse to this is Lemma 6.5. but with the relative notion $\ll$ in place of compactness itself: if $A_{n} \phi \Leftrightarrow \top$ then $\beta^{n} \ll \phi$ in the sense of Definition 2.1(b). For suppose that $\phi \leq \bigvee_{s} \theta_{s}$, so $\top \Leftrightarrow A_{n} \phi \Rightarrow A_{n} \bigvee_{s} \theta_{s} \Leftrightarrow \bigvee_{s} A_{n} \theta_{s}$. Then ${ }^{5}$ $A_{n} \theta_{s} \Leftrightarrow \top$ for some $s$, so $\beta^{n} \leq \theta_{s}$ by Lemma 6.4.

\footnotetext{
${ }^{5}$ This involves the same confusion of internally and externally defined joins as in Definition 5.2 cf. Remark 4.11
} 
Now suppose that a locale carries an effective basis in our sense. Then

$$
\phi=\exists n . A_{n} \phi \wedge \beta^{n} \equiv \bigvee\left\{\beta^{n} \mid A_{n} \phi\right\} \leq \bigvee\left\{\beta^{n} \mid \beta^{n} \ll \phi\right\} \leq \phi,
$$

in which the second join is directed, by Lemma 2.6. Hence the frame $L$ is continuous.

Remark 6.15. Notice that $\beta^{m} \leq \beta^{n}$ does not imply any relationship between $n, m \in N$, because the function $\beta^{(-)}: N \rightarrow L$ need not be injective. This is the reason why $A_{n}$ need not be exactly $\lambda \phi . \beta^{n} \ll \phi, c f$. Remark 1.8 .

Proposition 6.16. A locale is stably locally compact iff it has a lattice filter basis.

Proof. $[\Rightarrow]$ Let $A_{n}=\lambda \phi . \beta^{n} \ll \phi$ as before. $[\Leftarrow]$ As we have an $\vee$-basis, the basis expansion is a directed join, to which we may apply the definition of $\beta^{m} \ll \phi$. In this case there is some $n$ with $\beta^{m} \leq \beta^{n}$ and $A_{n} \phi \Leftrightarrow \top$, so $\beta^{m} \ll \top$. Also, if $\beta^{m} \ll \phi$ and $\beta^{m} \ll \psi$ then, for some $p, q$,

$$
\beta^{m} \leq \beta^{p}, \quad \beta^{m} \leq \beta^{q}, \quad A_{p} \phi \Leftrightarrow \top \text { and } A_{q} \psi \Leftrightarrow \top
$$

so $\beta^{m} \leq \beta^{p} \wedge \beta^{q} \equiv \beta^{p \star q}$. As we have a filter $\wedge$-basis, $A_{p \star q} \phi \wedge A_{p \star q} \psi \Leftrightarrow A_{p \star q}(\phi \wedge \psi) \Leftrightarrow \top$. Hence, with $n=p \star q, \beta^{m} \leq \beta^{n}$ and $A_{n}(\phi \wedge \psi) \Leftrightarrow \top$, so $\beta^{m} \ll \phi \wedge \psi$.

Remark 6.17. In summary, the dual basis $A_{n} \phi$ essentially says that there is a compact subobject $K^{n}$ lying between $\beta^{n}$ and $\phi$, but $K^{n}$ seems to play no actual role itself, and the localic definition in terms of $\ll$ makes it redundant, $c f$. Proposition 2.2 Nevertheless, each definition actually has its technical advantages:

(a) in the localic one, $\beta$ ranges over a lattice, but $\uparrow \beta$ need not be a filter;

(b) in the spatial one we have filters, but the basis need only be indexed by a semilattice.

Effective bases in our sense can be made to behave in either fashion, though we shall only consider lattice bases ( $c f$. the localic situation) in this paper (Remark 17.6] sketches how filter bases may be obtained). Stably locally compact objects have lattice filter bases, whose properties will be improved in Proposition 12.14 to take advantage of the intersections of compact subobjects.

\section{SigmA-SPLit SUBOBJECTS}

A basis for a vector space is exactly (the data for) an isomorphism with $\mathbb{R}^{N}$, where $N$ is the dimension of the space. It is not important for the analogy that the field of scalars be $\mathbb{R}$, or that the dimension be finite. The significance of $\mathbb{R}^{N}$ is that it carries a standard structure (in which the $n$th basis vector has a 1 in the $n$th co-ordinate and 0 elsewhere), which is transferred by the isomorphism to the chosen structure on the space under study. The standard object in our case is the object $\Sigma^{N}$ (or the corresponding algebra $\Sigma^{\Sigma^{N}}$ ), for which Axiom 4.10 defined a basis.

Bases for lattices are actually more like spanning sets than (linearly independent) bases for vector spaces, since we may add unions of members to the basis as we please, as we do in Lemma 8.4 below. Consequently, instead of isomorphisms with the standard structure, we 
have $\Sigma$-split embeddings $X \longrightarrow \Sigma^{N}$. We shall see that these embeddings capture several well known constructions involving $\mathbb{R}$ and locally compact objects.

Definition 7.1. $i: X \longrightarrow Y$ is a $\Sigma$-split subobject if (it is the equaliser of some pair [B] and) there is a map $I: \Sigma^{X} \rightarrow \Sigma^{Y}$ such that $\Sigma^{i} \cdot I=\mathrm{id}_{\Sigma^{X}}$. Using Notation 5.13, we write
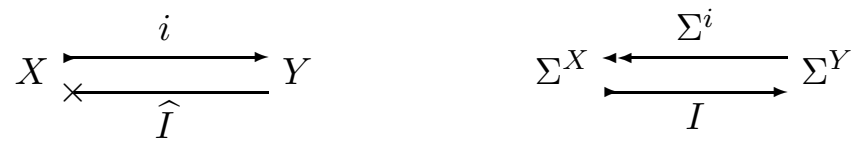

The effect of this is that $X$ carries the subspace topology inherited from $Y$, in a canonical way: for an open subobject $\phi$ of $X$, it provides a particular open subobject $I \phi$ of $Y$ for which the restriction $\Sigma^{i}(I \phi)$ is $\phi$.

In the case where $X$ is an open subobject of $Y$ (Lemma 3.8), $I \phi \equiv \exists_{i} \phi$ is the least such extension, whilst for a closed subobject, $I \phi \equiv \forall_{i} \phi$ is the greatest one, but in general it need not be either of these.

The computational significance of $\Sigma$-split embeddings is that any observation (computation of type $\Sigma$ ) on the subobject extends canonically, though not uniquely, to the whole object.

Warning 7.2. Let $Y \rightrightarrows Z$ be a parallel pair of continuous functions between locally compact sober spaces and $X \equiv\{y: Y \mid f y=g y: Z\}$ the subset of points that satisfy the equation. We give $X$ the subspace topology, i.e. its open subspaces are the restrictions of those of $Y$. This is the equaliser $X \longmapsto Y \rightrightarrows Z$ in the category of sober topological spaces, but $X$ need not be locally compact (or have an effective basis). Equalisers also exist in the category of locales, but again need not be locally compact, or even spatial.

$\Sigma$-split subspaces are therefore a very special case.

Remark 7.3. For this reason, the formulation of ASD that we gave in Axiom 3.3(c) does not provide general equalisers. It formally adjoins [B, $\S \S 4-6]$ a $\Sigma$-split subobject $i: X \equiv$ $\{Y \mid E\} \longmapsto Y$ of $Y$ with $I: \Sigma^{X} \triangleleft \Sigma^{Y}$ such that $\Sigma^{i} \cdot I=\mathrm{id}_{\Sigma^{X}}$ and $I \cdot \Sigma^{i}=E$, given any endomorphism $E: \Sigma^{Y} \rightarrow \Sigma^{Y}$ that satisfies the appropriate equation. We shall consider the formulation of this equation in Section [10. The $\lambda$-calculus developed in [B, $\S \S 8-10]$ allows arbitrarily complicated combinations of $\Sigma$-split subobjects and exponentials, but in this section and the next we reduce them to $\Sigma$-split subobjects of $\Sigma^{N}$.

The following results link three $(\mathrm{d}-\mathrm{f})$ of the abstract characterisations of local compactness in the Introduction.

Lemma 7.4. Any object $X$ that has an effective basis $\left(\beta^{n}, A_{n}\right)$ indexed by $N$ is a $\Sigma$-split subobject of $\Sigma^{N}$.

Proof. Using the basis $\left(\beta^{n}, A_{n}\right)$, define

$$
\begin{array}{ll}
i: X \rightarrow \Sigma^{N} & \text { by } \quad x \mapsto \xi \equiv \lambda n . \beta^{n} x \\
I: \Sigma^{X} \rightarrow \Sigma^{\Sigma^{N}} & \text { by } \quad \phi \mapsto \Phi \equiv \lambda \xi . \exists n . A_{n} \phi \wedge \xi n .
\end{array}
$$

Then $\Sigma^{i}(I \phi)=\lambda x .(I \phi)(i x)=\lambda x . \exists n . A_{n} \phi \wedge \beta^{n} x=\phi$. We also recover

$$
x=\operatorname{focus}\left(\exists n . A_{n} \wedge \xi n\right) \text {. }
$$

Incidentally, notice the use of letters here: we write $x: X, \phi: \Sigma^{X}$ and $F: \Sigma^{\Sigma^{X}}$ for the object under study, but $n: N, \xi: \Sigma^{N}$ and $\Phi: \Sigma^{\Sigma^{N}}$ for its basis, the idea being that $x$ and $\phi$ are represented by $\xi$ and $\Phi$ under the embedding. 
Conversely, any $\Sigma$-split subobject inherits the basis of the ambient object, using the inverse images of the basic open subobjects along $i: X \longrightarrow Y$. However, for the compact subobjects, we use their direct images along the "second class" map $\widehat{I}: Y \longrightarrow X$, in the sense of Remark 5.14. Since $I$ need not preserve meets, nor need the modal operator $\Sigma^{I} A \equiv A \cdot I$. This is why we find bases in which $A_{n}$ need not preserve $\top$ and $\wedge$.

Lemma 7.5. Let $\left(\beta^{n}, A_{n}\right)$ be an effective basis for $Y$ and $i: X \longrightarrow Y$ a $\Sigma$-split subobject. Then $\left(\Sigma^{i} \beta^{n}, \Sigma^{I} A_{n}\right)$ is an effective basis for $X$. If an $\vee$-or $\wedge$-basis was given, the result is one too. If $A_{n}$ is a filter and I preserves $\top$ and $\wedge$ then $\Sigma^{I} A_{n}$ is also a filter.

In other words, $\Sigma$-split subobjects of locally compact objects are again locally compact.

Proof. For $\phi: \Sigma^{X}, I \phi: \Sigma^{Y}$ has basis decomposition

$$
I \phi \Leftrightarrow \exists n . A_{n}(I \phi) \wedge \beta^{n} \equiv \exists n .\left(\Sigma^{I} A_{n}\right) \phi \wedge \beta^{n} .
$$

Since $\Sigma^{i}$ is a homomorphism, it preserves scalars, $\wedge$ and $\exists$, so

$$
\phi=\Sigma^{i}(I \phi)=\Sigma^{i}\left(\exists n . A_{n}(I \phi) \wedge \beta^{n}\right)=\exists n . A_{n}(I \phi) \wedge \Sigma^{i} \beta^{n} .
$$

Corollary 7.6. An object has an effective basis iff it is a $\Sigma$-split subobject of some $\Sigma^{N}$.

In the extension of ASD, we shall take this as the definition of a locally compact object. Proof. The Scott principle (Axiom 4.10) defined a basis on $\Sigma^{N}$.

These two constructions are not inverse: given an $N$-indexed effective basis on $X$, we obtain a $\Sigma$-split embedding $X \longmapsto \Sigma^{N}$, and then a basis indexed by $\operatorname{Fin}(N)$. In Lemma 15.6 we shall want to recover the original, $N$-indexed, basis.

Lemma 7.7. An embedding $X \longrightarrow \Sigma^{N}$ arises from a basis according to Lemma 7.4 iff each I $\phi$ preserves joins. It's then a filter basis iff, for each $n, \lambda \phi . I \phi\{n\}$ also preserves finite meets.

Proof. For any basis, $\xi \mapsto I \phi \xi \equiv \exists n . A_{n} \phi \wedge \xi n$ preserves joins. Conversely, with

$$
\beta^{n} \equiv \lambda x . i x n \quad \text { and } \quad A_{n} \equiv \lambda \phi . I \phi\{n\}
$$

we recover $\phi x \equiv(I \phi)(i x) \Leftrightarrow \exists n . I \phi\{n\} \wedge i x n \Leftrightarrow \exists n . A_{n} \phi \wedge \beta^{n} x$ so long as $I \phi$ preserves the join $i x=\exists n$. ixn $\wedge\{n\}$.

In the rest of this section we consider the classical interpretations of the $\Sigma$-split embedding that arises from an effective basis, starting with traditional topology. Recall from [Joh82, Theorem II 1.2] that the free frame on $N$ is $\Upsilon K N$ (the lattice of upper subsets of $\mathrm{K} N$ ), and that this is isomorphic to the lattice of Scott-open subsets of the powerset $\mathcal{P}(N)$.

Theorem 7.8. Let $X$ be a locally compact sober space with $N$-indexed basis $\left(U^{n}, K^{n}\right)$. Then $X$ is a $\Sigma$-split subspace of $\mathcal{P}(N)$.

Proof. The embedding in Lemma 7.4 takes

$$
\begin{array}{lll}
x \in X & \text { to } & \left\{n \mid x \in U^{n}\right\} \in \mathcal{P}(N) \\
V \subset X & \text { to } & \left\{\ell \mid \exists n \in \ell . K^{n} \subset V\right\} \in \Upsilon K N .
\end{array}
$$

The second map, $I$, is Scott-continuous because it takes $\bigcup_{s} V_{s}$ to

$$
\left\{\ell \mid \exists n \in \ell . K^{n} \subset \bigcup_{s} V_{s}\right\}=\left\{\ell \mid \exists n \in \ell . \exists s . K^{n} \subset V_{s}\right\}=\bigcup_{s}\left\{\ell \mid \exists n \in \ell . K^{n} \subset V_{s}\right\} .
$$


The composite $\Sigma^{i} \cdot I$ takes $V \subset X$ to $\bigcup\left\{\bigcap_{n \in \ell} U^{n} \mid \exists n \in \ell . K^{n} \subset V\right\}$, and by Definition 1.1. this contains $V=\left\{x \mid \exists n . x \in U^{n} \subset K^{n} \subset V\right\}$. For the converse, if $x \in \Sigma^{i}(I V)$ then $\exists \ell . \forall n \in \ell . x \in U^{n} \wedge \exists m \in \ell . K^{n} \subset V$, so $\exists n . x \in U^{n} \subset K^{n} \subset V$.

Example 7.9. A compact Hausdorff space has a basis determined by a family of disjoint pairs $\left(U^{n} \not V_{n}\right)$ of open subspaces. In this case, the embedding is

$$
\begin{aligned}
& x \mapsto\left\{n \mid x \in U^{n}\right\} \\
& W \mapsto\left\{\ell \mid \exists n \in \ell . V_{n} \cup W=X\right\}
\end{aligned}
$$

Consider in particular the embedding of $\mathbb{R}$ in $\Sigma^{N}$, where $N$ indexes a basis of open and closed intervals (Examples 1.4 and 6.10). This is closely related to one of the first examples that Dana Scott used to show how continuous lattices could be used as a model of computation Sco70.

Definition 7.10. The lattice of intervals, $\mathbb{I R}^{\top}$, of $\mathbb{R}$ is the set of convex closed subspaces (including the empty one), ordered by reverse inclusion, and given the Scott topology. The domain of intervals, $I \mathbb{R} \subset I^{\top}$, consists of the inhabited such subspaces. Amongst these, points of $\mathbb{R}$ are identified with the maximal elements. Classically, the members of IR are of the form $[r \pm \delta]$, with $r \in \mathbb{R}$ and $0 \leq \delta \leq \infty$.

Remark 7.11. In ASD, closed subobjects under reverse inclusion correspond to their coclassifiers under the (forward) intrinsic order, and to the "complementary" open subobjects under inclusion. When the closed subobject is convex, the open one is $\delta \vee v$, where $\delta$ is lower and $v$ upper in the arithmetical order, so the pair $(\delta, v)$ is almost a Dedekind cut $[\mathbb{I}]$, except that it is disjoint but need not be "located". It has this last property exactly when the closed subobject is a singleton.

Intervals $[p, q]$ or $[r \pm \delta]$ with specified endpoints are of this form, but (in ASD and other constructive forms of analysis), not every inhabited convex closed bounded subobject need have endpoints. It does so iff it is also overt [J]. In particular, a directed join in IR of subobjects with endpoints need not itself have them.

It is, however, not necessary to appreciate this issue in order to see the relationship between $I \mathbb{R}$ and our representation of $\mathbb{R}$ as a $\Sigma$-split subobject of $\Sigma^{N}$.

Proposition 7.12. In LKSp, $\mathbb{R} \longrightarrow \mathrm{I} \longrightarrow \mathbb{R}^{\top} \rightleftarrows \Sigma^{N}$, where $N$ is the set of pairs, written $\langle q \pm \epsilon\rangle$, with $\epsilon>0$ and $q$ rational.

(Recall that the $\longrightarrow$ indicates a closed inclusion.)

Proof. The embedding takes $r \in \mathbb{R}$ to $[r \pm 0]$ and then to $\lambda\langle q \pm \epsilon\rangle . r \in(q \pm \epsilon)$, which is (the exponential transpose of ) a continuous function. The retraction is defined by intersection:

$$
\begin{array}{lll}
\mathrm{IR}^{\top} & \longmapsto & \Sigma^{N} \\
C & \longmapsto & \lambda\langle q \pm \epsilon\rangle \cdot C \subset(q \pm \epsilon) \\
{[r \pm \delta]} & \longmapsto & \lambda\langle q \pm \epsilon\rangle \cdot q-\epsilon<r-\delta \leq r+\delta<q+\epsilon \\
\emptyset & \longmapsto & \lambda\langle q \pm \epsilon\rangle \cdot \top \\
\mathbb{R} & \longmapsto & \lambda\langle q \pm \epsilon\rangle \cdot\left(\epsilon={ }_{N} \infty\right) \\
\bigcap_{\phi\langle q \pm \epsilon\rangle}[q \pm \epsilon] & \longmapsto
\end{array}
$$


Any compact interval of the $T_{1}$ space $\mathbb{R}$ is saturated in the sense of Lemma [5.15] It is therefore the intersection of its open neighbourhoods, amongst which open intervals suffice. Hence the composite is $\mathrm{IR}^{\top} \rightarrow \Sigma^{N} \rightarrow \mathrm{IR}^{\top}$ is the identity.

The projection $\mathbb{I R}^{\top} \longleftarrow \Sigma^{N}$ is Scott-continuous because it clearly takes directed unions of sets of codes to codirected intersections of compact subspaces. Classically, Lemma [5.17] showed that such intersections correspond to unions of neighbourhood filters, whilst in ASD they correspond to the unions of the complementary open subobjects $\delta \vee v$. Hence the inclusion $\mathbb{I}^{\top} \longrightarrow \Sigma^{N}$ is also Scott-continuous.

The inverse image of $\top$ under $I^{\top} \longleftarrow \Sigma^{N}$ is the open subobject classified by the inconsistency predicate

$$
\ln \operatorname{Con}(\phi) \equiv \exists\left\langle q_{1} \pm \epsilon_{1}\right\rangle\left\langle q_{2} \pm \epsilon_{2}\right\rangle .\left(q_{1}+\epsilon_{1}<q_{2}-\epsilon_{2}\right) \wedge \phi\left\langle q_{1} \pm \epsilon_{1}\right\rangle \wedge \phi\left\langle q_{2} \pm \epsilon_{2}\right\rangle .
$$

The complementary closed subobject of $\Sigma^{N}$ is of course not classified, as it's not open, but when we restrict attention to its (overt discrete collection of) finite elements, we find that consistency is characterised by the decidable formula

so

$$
\begin{gathered}
\operatorname{Con}(\ell) \equiv \exists x: \mathbb{Q} . \forall\langle q \pm \epsilon\rangle \in \ell . x \in\langle q \pm \epsilon\rangle, \\
\ln \operatorname{Con}(\lambda\langle q \pm \epsilon\rangle \cdot\langle q \pm \epsilon\rangle \in \ell)=\neg \operatorname{Con}(\ell) .
\end{gathered}
$$

Remark 7.13. The idea behind the domain of intervals is not hard to generalise. Indeed, we may embed any locally compact sober space as a subspace of its continuous preframe of compact saturated subspaces (Theorem [5.18), each point being represented by its saturation in the sense of Lemma 5.15. That the image consists of the maximal points (excluding $\emptyset)$ plainly depends on starting with a $T_{1}$-space, so can't be an essential feature of the construction.

Another interesting aspect of the general construction is the decidable consistency predicate on finite elements. Scott developed these into what became a standard form of domain theory $\mathrm{Sco} 82$. The subobject of $\Sigma^{N}$ that is determined by the consistent elements is closed, but also overt $[\mathrm{F}]$.

We shall develop our own construction of the real line via Dedekind cuts, and also discuss the interval domain further, in $[\mathbf{I}]$.

Now we give the localic version of Theorem 7.8 This result is the most efficient way of establishing the connection between locally compact locales and ASD.

Theorem 7.14. Let $L$ be any $N$-based continuous distributive lattice. Then there is a frame homomorphism $H$ and a Scott-continuous function $I$ with $H \cdot I=\mathrm{id}_{L}$, as shown:

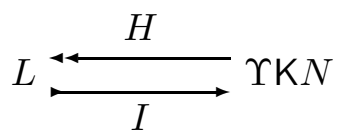

Conversely, any lattice $L$ that admits such a pair of functions is continuous and distributive.

Proof. $[\Rightarrow]$ Let $\left(\beta^{n}\right)$ be a basis for the continuous lattice $L$ and $A_{n} \equiv \lambda \phi .\left(\beta^{n} \ll \phi\right)$. Let $H: \Upsilon \mathrm{K} N \rightarrow L$ be the unique frame homomorphism that extends $\beta^{(-)}: N \rightarrow L$, so

$$
\text { for } \mathcal{U} \in \Upsilon K N, \quad H \mathcal{U}=\bigvee_{\ell \in \mathcal{U}} \bigwedge_{n \in \ell} \beta^{n} \in L,
$$


and define $I: L \rightarrow \Upsilon K N$ by $I \phi=\left\{\ell \mid \exists n \in \ell . \beta^{n} \ll \phi\right\}$. This is Scott-continuous by a similar argument to that in Theorem 7.8, with $\beta^{n} \preccurlyeq \phi_{s}$ instead of $K^{n} \subset V_{s}$. Then

$$
\begin{aligned}
\beta^{n} \ll H(I \phi) & \Longleftrightarrow \exists \ell \cdot \beta^{n} \ll \bigwedge_{m \in \ell} \beta^{m} \wedge \exists m \in \ell \cdot \beta^{m} \ll \phi \\
& \Longleftrightarrow \exists m . \beta^{n} \ll \beta^{m} \ll \phi \Longleftrightarrow \beta^{n} \ll \phi,
\end{aligned}
$$

from which we deduce $H(I \phi)=\phi$, because $\left(\beta^{n}\right)$ is a basis.

$[\Leftarrow]$ Conversely, if such a diagram exists then $I \cdot H$ is a Scott-continuous idempotent on a continuous lattice, so its splitting $L$ is also continuous [Joh82, Lemma VII 2.3]. As $H$ preserves joins, it has a right adjoint, $H \dashv R$, so id $\leq R \cdot H \equiv j=j \cdot j$, and $R$ preserves meets but not necessarily directed joins. Since $H$ also preserves finite meets, so does $j$, and this is a nucleus in the sense of locale theory [Joh82, Section II 2.2], so its splitting $L$ is a frame.

Any locally compact locale is therefore determined by a Scott-continuous idempotent $\mathcal{E}$ on $\Upsilon K N$. It is not just an idempotent, however, since the surjective part of its splitting must be a frame homomorphism. Since the latter preserves $T$ and $\perp$ by monotonicity, and $\bigvee$ as $\mathcal{E}$ is Scott-continuous, it is enough to identify the condition on $\mathcal{E}$ the ensures preservation of the two binary lattice connectives, which we may treat exactly alike. (This is where Remark 3.4 came from.)

Lemma 7.15. Let $I$ and $H$ be monotone functions between two semilattices, with $H \cdot I=$ id. Then $H$ preserves $\wedge$ iff $E \equiv I \cdot H$ satisfies the equation $E(\phi \wedge \psi)=E(E \phi \wedge E \psi)$.

Proof. If $H$ preserves $\wedge$ then

$$
\begin{aligned}
E(\phi \wedge \psi) & \equiv I(H(\phi \wedge \psi)) \\
& =I(H \phi \wedge H \psi) \\
& =I(H \cdot I \cdot H \phi \wedge H \cdot I \cdot H \psi) \\
& =I \cdot H(I \cdot H \phi \wedge I \cdot H \psi) \\
& \equiv E(E \phi \wedge E \psi)
\end{aligned}
$$

For the converse, note first that we have $H(\phi \wedge \psi) \leq H \phi \wedge H \psi$ and $I\left(\phi^{\prime} \wedge \psi^{\prime}\right) \leq I \phi^{\prime} \wedge I \psi^{\prime}$ by the definition of $\wedge$. Then

$$
\begin{array}{rlr}
I(H \phi \wedge H \psi) & =E(I(H \phi \wedge H \psi)) & I=I \cdot H \cdot I=E \cdot I \\
& \leq E(I \cdot H \phi \wedge I \cdot H \psi) & \text { above } \\
& =E(E \phi \wedge E \psi) & E=I \cdot H \\
& =E(\phi \wedge \psi) & \text { hypothesis } \\
& =I \cdot H(\phi \wedge \psi) & E=I \cdot H \\
H \phi \wedge H \psi & \leq H(\phi \wedge \psi) & H \cdot I=\mathrm{id}
\end{array}
$$

so $H(\phi \wedge \psi) \leq H \phi \wedge H \psi \leq H(\phi \wedge \psi)$.

Corollary 7.16. Any $N$-based locally compact locale is determined by a Scott-continuous endofunction of the frame $\Upsilon \mathrm{K} N$ (or by a locale endomorphism of $\Sigma^{\Sigma^{N}}$ ) such that

$$
E(\phi \wedge \psi)=E(E \phi \wedge E \psi) \text { and } E(\phi \vee \psi)=E(E \phi \vee E \psi)
$$


We now have to concentrate on the logical development within abstract Stone duality, and will only return to the connection with traditional topology in Section 17 The first task is to show that every definable object has an effective basis, and is therefore a $\Sigma$-split subobject of $\Sigma^{N}$. Such subobjects are determined by idempotents $\mathcal{E}$ on $\Sigma^{\Sigma^{N}}$ satisfying the equation that we have just identified, along with its counterpart for $\vee$. However, even that characterisation depends on the use of bases (Lemma 10.7f).

\section{EVERY DEFINABLE OBJECT HAS A BASIS}

In Section [6] we justified the notion of effective basis in the classical models, i.e. for locally compact sober spaces and locales. This section considers the term model, showing by structural recursion that every definable object has an effective basis. We have already dealt with the base cases $\left(\mathbb{N}, \Sigma^{\mathbb{N}}\right)$ in Example 6.7, and with $\Sigma$-split subobjects in Lemma 7.5. So we consider binary products first (leaving the reader to define the $\mathbf{1}$-indexed basis for $\mathbf{1}$ ), but devote most of the section to the exponential $\Sigma^{X}$.

Lemma 8.1. If $X$ and $Y$ have effective bases then so does $X \times Y$, given by Tychonov rectangles.

Proof. We are given $\left(\beta^{n}, A_{n}\right)$ and $\left(\gamma^{m}, D_{m}\right)$ on $X$ and $Y$. Then, on $X \times Y$, we define

$$
\begin{aligned}
& \epsilon^{(n, m)} \equiv \lambda x y \cdot \beta^{n} x \wedge \gamma^{m} y \\
& F_{(n, m)} \equiv \lambda \theta: \Sigma^{X \times Y} \cdot D_{m}\left(\lambda y \cdot A_{n}(\lambda x \cdot \theta(x, y))\right),
\end{aligned}
$$

Then

$$
\begin{aligned}
\theta(x, y) & \Leftrightarrow \exists n \cdot A_{n}\left(\lambda x^{\prime} \cdot \theta\left(x^{\prime}, y\right)\right) \wedge \beta^{n} x \\
& \Leftrightarrow \exists n m \cdot D_{m}\left(\lambda y^{\prime} \cdot A_{n}\left(\lambda x^{\prime} \cdot \theta\left(x^{\prime}, y^{\prime}\right)\right)\right) \wedge \gamma^{m} y \wedge \beta^{n} x \\
& \Leftrightarrow \exists(n, m) \cdot F_{(n, m)} \theta \wedge \epsilon^{(n, m)}(x, y)
\end{aligned}
$$

Notice that the formula for $F_{(n, m)}$ is not symmetrical in $X$ and $Y$, though we have learned to expect properties of binary products to be asymmetrical and problematic [A]. In fact, if $A_{n}$ and $D_{m}$ are filter bases, we have another example of the same problem that held us back in Proposition 5.11, along with the core of its solution.

Lemma 8.2. If $A: \Sigma^{\Sigma^{X}}$ and $D: \Sigma^{\Sigma^{Y}}$ both preserve either $\top$ and $\wedge$ or $\perp$ and $\vee$ then

$$
A(\lambda x . D(\lambda y . \theta x y)) \Leftrightarrow D(\lambda y . A(\lambda x . \theta x y))
$$

whenever $\theta: \Sigma^{X \times Y}$ is a finite union of rectangles.

Proof. Applying the Phoa principle (Axiom 3.6) to a single rectangle,

$$
\begin{aligned}
& A(\lambda x . D(\lambda y . \phi x \wedge \psi y)) \\
& \Leftrightarrow A(\lambda x . D \perp \vee \phi x \wedge D \psi) \quad \text { Phoa for } D \text { wrt } \phi x \\
& \Leftrightarrow A(\lambda x . \phi x \wedge D \psi) \vee(D \perp \wedge A \top) \quad \text { Phoa for } A \text { wrt } D \perp \\
& \Leftrightarrow A \perp \vee(D \psi \wedge A \phi) \vee(D \perp \wedge A \top) \quad \text { Phoa for } A \text { wrt } D \psi \text {. }
\end{aligned}
$$

This would have the required symmetry if we had

$$
A(D \perp) \equiv A \perp \vee(D \perp \wedge A \top) \Leftrightarrow(A \perp \wedge D \top) \vee D \perp \equiv D(A \perp) .
$$

If $A \top \Leftrightarrow \top \Leftrightarrow D \top$ then both sides are $A \perp \vee D \perp$, whilst if $A \perp \Leftrightarrow \perp \Leftrightarrow D \perp$ then they are both $\perp$. Under either hypothesis, the lattice dual argument shows the similar result

$$
A(\lambda x . D(\lambda y . \phi x \vee \psi y)) \Leftrightarrow D(\lambda y . A(\lambda x . \phi x \vee \psi y))
$$


for a cross. The result uses equational induction [E, §2], but we shall just illustrate this with the case where $\theta$ is a union of three rectangles,

$$
\theta x y \Leftrightarrow\left(\phi_{1} x \wedge \psi_{1} y\right) \vee\left(\phi_{2} x \wedge \psi_{2} y\right) \vee\left(\phi_{3} x \wedge \psi_{3} y\right) .
$$

If $A$ and $D$ preserve $\perp$ and $\vee$ then $A D \theta$ is also a union of three terms, to each of which the first part applies.

We may also use distributivity of $\vee$ over $\wedge$ to re-express the union $\theta$ as an intersection of eight crosses,

$$
\begin{aligned}
\theta x y \Leftrightarrow & \left(\phi_{1} x \vee \phi_{2} x \vee \phi_{3} x\right) \wedge\left(\phi_{1} x \vee \phi_{2} x \vee \psi_{3} y\right) \wedge \\
& \left(\phi_{1} x \vee \psi_{2} y \vee \phi_{3} x\right) \wedge\left(\phi_{1} x \vee \psi_{2} y \vee \psi_{3} y\right) \wedge \\
& \left(\psi_{1} y \vee \phi_{2} x \vee \phi_{3} x\right) \wedge\left(\psi_{1} y \vee \phi_{2} x \vee \psi_{3} y\right) \wedge \\
& \left(\psi_{1} y \vee \psi_{2} y \vee \phi_{3} x\right) \wedge\left(\psi_{1} y \vee \psi_{2} y \vee \psi_{3} y\right) .
\end{aligned}
$$

So if $A$ and $D$ preserve $\wedge$ then $A D \theta$ is a conjunction of eight factors, to each of which the second part applies, for example with $\phi=\phi_{1} \vee \phi_{2}$ and $\psi=\psi_{3}$. In both cases, $A D \theta \Leftrightarrow D A \theta$ as required.

Remark 8.3. This still awaits Theorem 9.6 on Scott continuity to extend finite unions of rectangles to infinite ones, but once we have that we may draw the corollaries that

(a) if $X$ and $Y$ have filter bases then Lemma 8.1 provides a filter basis for $X \times Y$, which is symmetrical in $X$ and $Y$;

(b) Proposition 5.11yields a bijection between closed and compact subobjects of a compact Hausdorff object. Moreover, despite the other problems discussed in Section [5, preserving finite meets is enough to characterise $\square$ modal operators.

We shall need to be able to turn any effective basis into a $\vee$-basis, which we do in the obvious way using finite unions of basic open subobjects. The corresponding unions of compact subobjects give rise to conjunctions of As by Lemma 5.7(c) Unfortunately, the result is topologically rather messy, both for products and for objects such as $\mathbb{R}$ that we want to construct directly.

Lemma 8.4. If $X$ has an effective basis indexed by $N$ then it also has a $\vee$-basis indexed by $\operatorname{Fin}(N)$. If we were given a filter basis, the result is one too.

Proof. Given any basis $\left(\beta^{n}, A_{n}\right)$, define

$$
\gamma^{\ell} \equiv \lambda x . \exists n \in \ell . \beta^{n} x \quad D_{\ell} \equiv \lambda \phi . \forall n \in \ell . A_{n} \phi .
$$

Then $\phi=\exists n . A_{n} \phi \wedge \beta^{n} \leq \exists \ell . D_{\ell} \phi \wedge \gamma^{\ell}$ using singleton lists. Conversely,

$$
\begin{aligned}
\exists \ell . D_{\ell} \phi \wedge \gamma^{\ell} & =\exists \ell .\left(\forall n \in \ell \cdot A_{n} \phi\right) \wedge\left(\exists m \in \ell \cdot \beta^{m}\right) \\
& =\exists \ell \cdot \exists m \in \ell \cdot\left(\forall n \in \ell \cdot A_{n} \phi\right) \wedge \beta^{m} \\
& \leq \exists \ell \cdot \exists m \in \ell \cdot A_{m} \phi \wedge \beta^{m}=\phi .
\end{aligned}
$$

Then $\left(\gamma^{\ell}, D_{\ell}\right)$ is a $\vee$-basis, using list concatenation for + . The imposed order $\preccurlyeq$ on $\operatorname{Fin}(N)$ is list or subset inclusion,

$$
\left(\ell \preccurlyeq \ell^{\prime}\right) \equiv\left(\ell \subset \ell^{\prime}\right) \equiv \forall n \in \ell . n \in \ell^{\prime} .
$$

Finally, if the $A_{n}$ were filters then so are the $D_{\ell}$, since $\forall m \in \ell$ preserves $\wedge$ and $\top$. 
Remark 8.5. Computationally, it may be better to see this as an embedding $i: X \longmapsto \Sigma^{\Sigma^{N}}$ rather than into $\Sigma^{\mathrm{Fin} N}$, as this gives a very simple representation of the basis and dual basis. Using the notation $\pi_{\ell} \equiv \lambda n . n \in \ell$ and $[\ell] \equiv \forall n \in \ell$. $\psi n$ from [E], which also provides a fixed-point equation for $\exists \ell$, this is:

$$
\begin{aligned}
i x & =\lambda \xi . \exists n \cdot \beta^{n} x \wedge \xi n \\
I \phi & =\lambda \Phi \cdot \exists \ell . \Phi(\lambda n . n \in \ell) \wedge \forall n \in \ell \cdot A_{n} \phi \\
\gamma^{\ell} x & \Leftrightarrow \exists n \in \ell \cdot \beta^{n} x \Leftrightarrow i x(\lambda n . n \in \ell) \Leftrightarrow i x \pi_{\ell} \\
D_{\ell} \phi & \Leftrightarrow \forall n \in \ell \cdot A_{n} \phi \Leftrightarrow I \phi(\lambda \psi \cdot \forall n \in \ell . \psi n) \Leftrightarrow I \phi[\ell] \\
\phi x & \Leftrightarrow \exists \ell . I \phi[\ell] \wedge i x \pi_{\ell}
\end{aligned}
$$

Lemma 8.6. If an $\wedge$-basis was given, Lemma 8.4 yields a lattice basis.

Proof. We are given $\beta^{1}=\top$ and $\beta^{n} \wedge \beta^{m}=\beta^{n \star m}$.

Let $\ell \star \ell^{\prime}$ be the list (it doesn't matter in what order) of all $n \star m$ for $n \in \ell$ and $m \in \ell^{\prime}$. In functional programming notation, this is

$$
\ell \star \ell^{\prime} \equiv \text { flatten }\left(\operatorname{map} \ell\left(\lambda n . \operatorname{map} \ell^{\prime}(\lambda m . n \star m)\right)\right),
$$

where map applies a function to each member of a list, returning a list, and flatten turns a list of lists into a simple list. Categorically, map is the effect of the functor List $(-)$ on morphisms, and flatten is the multiplication for the List monad. Using the corresponding notions for $\mathrm{K}(-)$, « can similarly be defined for finite subsets instead.

Now let

$$
\beta^{\ell \star \ell^{\prime}} \equiv\left(\exists n \in \ell . \exists m \in \ell^{\prime} . \beta^{n \star m}\right)=\left(\gamma^{\ell} \wedge \gamma^{\ell^{\prime}}\right)
$$

by distributivity in $\Sigma^{X}$, whilst $\gamma^{\{1\}} \equiv \beta^{1}$ and $D_{\{1\}} \equiv A_{1}$ (where $\{1\}$ is the singleton list) serve for $\gamma^{1}$ and $D_{1}$. (This uses equational induction, [E, §2].)

Remark 8.7. By switching the quantifiers, we may similarly obtain $\wedge$-bases, and turn an $\checkmark$-basis into a lattice basis. In fact, this construction featured in the proof of Theorems 7.8 and 7.14. This time the filter property is not preserved, since if $A_{n} \phi$ means $K^{n} \subset U$ then $A_{\ell} \phi$ means that $\exists n \in \ell . K^{n} \subset U$, which is not the same as testing containment of a single compact subobject. Proposition 12.14 shows how to define an ^-basis for a locally compact object in which $A_{n \star m}$ actually captures the intersection of the compact subobjects.

Proposition 8.8. $N$ and $\Sigma^{N}$ have effective bases as follows:

$$
\begin{array}{llll}
N & \text { prime } & \beta^{n} \equiv\{n\} \equiv \lambda m .(n=m) & A_{n} \equiv \eta_{N}(n) \equiv \lambda \xi . \xi n \\
N & \text { filter } \vee & \beta^{\ell} \equiv \lambda m . m \in \ell & A_{\ell} \equiv \lambda \xi . \forall m \in \ell . \xi m \\
N & \text { lattice } & \beta^{L} \equiv \lambda m . \forall \ell \in L . m \in \ell & A_{L} \equiv \lambda \xi . \exists \ell \in L . \forall m \in \ell . \xi m \\
\Sigma^{N} & \text { prime } \wedge & B^{\ell} \equiv A_{\ell} & \mathcal{A}_{\ell} \equiv \eta_{\Sigma^{N}} \beta^{\ell} \equiv \lambda \Phi . \Phi(\lambda m . m \in \ell) \\
\Sigma^{N} & \text { filter lattice } & B^{L} \equiv A_{L} & \mathcal{A}_{L} \equiv \lambda \Phi . \forall \ell \in L . \Phi(\lambda m . m \in \ell)
\end{array}
$$

indexed by $n: N, \ell: \operatorname{Fin}(N)$ or $L: \operatorname{Fin}(\operatorname{Fin}(N))$. The interchange of sub-and superscripts means that we're reversing the imposed order on these indexing objects (Remark 4.12). 
The last of these also provides a basis for $\Sigma^{\Sigma^{N}}$, using Axiom 4.10 twice.

Lemma 8.9. $\Sigma^{\Sigma^{N}}$ has a $\operatorname{Fin}(\operatorname{Fin} N)$-indexed prime $\wedge$-basis with

$$
\mathcal{B}^{L} \equiv \mathcal{A}_{L} \equiv \lambda \Phi . \forall \ell \in L . \Phi \beta^{\ell} \quad \text { and } \quad \mathbb{A}_{L} \equiv \eta_{\Sigma^{2} N} A_{L} \equiv \lambda \mathcal{F} . \mathcal{F} A_{L},
$$

so, using $\Sigma^{3} N$ as a shorthand for a tower of exponentials, $\Sigma^{\Sigma^{\Sigma^{N}}} \equiv(((N \rightarrow \Sigma) \rightarrow \Sigma) \rightarrow \Sigma)$,

$$
\mathcal{F}: \Sigma^{3} N, \Phi: \Sigma^{\Sigma^{N}} \vdash \mathcal{F} \Phi \Leftrightarrow \exists L: \operatorname{Fin}(\operatorname{Fin} N) . \mathcal{F} A_{L} \wedge \forall \ell \in L . \Phi \beta^{\ell} .
$$

Proof. The prime $\wedge$-basis on $\Sigma^{N}$ makes $\Sigma^{\Sigma^{N}} \triangleleft \Sigma^{\mathrm{Fin}(N)}$ by

$$
\Phi \mapsto \lambda \ell . \Phi \beta^{\ell} \quad \text { and } \quad \lambda \xi . \exists \ell . \Psi \ell \wedge \forall n \in \ell . \xi n \longleftrightarrow \Psi,
$$

so $\Sigma^{3} N \triangleleft \Sigma^{2} \operatorname{Fin}(N)$ by

$$
\mathcal{F} \mapsto \lambda \Psi . \mathcal{F}(\lambda \xi . \exists \ell . \Psi \ell \wedge \forall n \in \ell . \xi n) \quad \text { and } \quad \lambda \Phi . \mathcal{G}\left(\lambda \ell . \Phi \beta^{\ell}\right) \longleftrightarrow \mathcal{G} .
$$

For any $\mathcal{F}: \Sigma^{3} N$ and $\Psi: \Sigma^{\operatorname{Fin}(N)}$, let $\mathcal{G}$ be obtained from $\mathcal{F}$ in this way, and use the prime $\wedge$-basis on $\Sigma^{\operatorname{Fin}(N)}$ :

$$
\begin{aligned}
\mathcal{G} \Psi & \Leftrightarrow \exists L: \operatorname{FinFin}(N) . \mathcal{G}(\lambda \ell . \ell \in L) \wedge \forall \ell \in L . \Psi \ell \\
& \Leftrightarrow \exists L . \mathcal{F}(\lambda \xi . \exists \ell .(\lambda \ell . \ell \in L) \ell \wedge \forall n \in \ell . \xi n) \wedge \forall \ell \in L . \Psi \ell \\
& \Leftrightarrow \exists L . \mathcal{F}(\lambda \xi . \exists \ell \in L . \forall n \in \ell . \xi n) \wedge \forall \ell \in L . \Psi \ell \\
& \Leftrightarrow \exists L . \mathcal{F} A_{L} \wedge \forall \ell \in L . \Psi \ell
\end{aligned}
$$

so $\mathcal{F}=\lambda \Phi . \mathcal{G}\left(\lambda \ell . \Phi \beta^{\ell}\right)=\lambda \Phi . \exists L . \mathcal{F} A_{L} \wedge \forall \ell \in L . \Phi \beta^{\ell}$.

Lemma 8.10. If an object $X$ has an effective basis $\left(\beta^{n}, A_{n}\right)$ then its topology $\Sigma^{X}$ has a prime lattice basis $\left(D^{L}, \lambda F . F \gamma_{L}\right)$, indexed by $L: \operatorname{Fin}(\operatorname{Fin} N)$, where

$$
\gamma_{L} \equiv \lambda x . \exists \ell \in L . \forall n \in \ell . \beta^{n} x \quad \text { and } \quad D^{L} \equiv \lambda \phi . \forall \ell \in L . \exists n \in \ell . A_{n} \phi .
$$

Proof. By Lemma 7.4 there is an embedding $i: X \longrightarrow \Sigma^{N}$ with $\Sigma$-splitting $I$ by

$$
i x \equiv \lambda n . \beta^{n} x \quad \text { and } \quad I \phi \equiv \Phi \equiv \lambda \xi . \exists n . A_{n} \phi \wedge \xi n, \quad \text { so } \quad \phi=\lambda x . \Phi(i x) .
$$

For $F: \Sigma^{\Sigma^{X}}$, let $\mathcal{F} \equiv F \cdot \Sigma^{i} \equiv \lambda \Psi . F(\lambda x . \Psi(i x)): \Sigma^{3} N$, so $\mathcal{F} \cdot I=F \cdot \Sigma^{i} \cdot I=F$. Then

$$
\begin{array}{rlr}
F \phi \Leftrightarrow \mathcal{F}(I \phi) & \Leftrightarrow \exists L . \mathcal{F} A_{L} \wedge \forall \ell \in L . I \phi \beta^{\ell} & \text { Lemma 8.9 } \\
& \Leftrightarrow \exists L . F\left(\Sigma^{i} A_{L}\right) \wedge \forall \ell \in L . I \phi \beta^{\ell} & \\
& \Leftrightarrow \exists L . F \gamma_{L} \wedge D^{L} \phi
\end{array}
$$

since the given formulae are $\gamma_{L}=\Sigma^{i} A_{L}$ and $D^{L}=\lambda \phi . \forall \ell \in L . I \phi \beta^{\ell}$.

Theorem 8.11. Every definable (or locally compact, cf. Remark 6.3) object $X$ has a lattice basis, indexed by $\operatorname{Fin}(\operatorname{Fin} \mathbb{N})$, and is a $\Sigma$-split subobject of $\Sigma^{\mathbb{N}}$. Moreover, $\Sigma^{X}$ is stably locally compact, as it has a prime lattice basis (cf. Proposition [6.16). 
Remark 8.12. This is a "normal form" theorem, and, like all such theorems, it can be misinterpreted. It is a bridge over which we may pass in either direction between $\lambda$-calculus and a discrete encoding of topology, not an intention to give up the very pleasant synthetic results that we saw in C. In particular, we make no suggestion that either arguments in topology or their computational interpretations need go via the list or subset representation (though JKM99 seems to have this in mind). Indeed, subsets may instead be represented by $\lambda$-terms [E]. It is simply a method of proof, and is exactly what we need to connect synthetic abstract Stone duality with the older lattice-theoretic approaches to topology, as we shall now show.

\section{BASIC COROLLARIES}

Making use of the availability of bases for all definable objects, this section establishes the basic properties that justify the claim that abstract Stone duality is an account of domain theory and general topology, at least in so far as its morphisms are continuous functions. We first prove something that was claimed in Remark 4.8 .

Proposition 9.1. Let $M$ be an overt discrete object with effective basis $\left(\beta^{n}, A_{n}\right)$ indexed by an overt discrete object $N$. Then $M$ is the subquotient of $N$ by an open partial equivalence relation.

Proof. Write $n \Vdash x$ for $n: N, x: M \vdash A_{n}\{x\} \wedge \beta^{n} x$ (using discreteness of $M$ ) and $N^{\prime}=\{n \mid \exists x . n \Vdash x\} \subset N$, which is open, using overtness.

Then, using the basis expansion of open $\{x\}$,

$$
\top \Leftrightarrow\left(x={ }_{M} x\right) \Leftrightarrow\{x\}(x) \Leftrightarrow \exists n . A_{n}\{x\} \wedge \beta^{n} x \Leftrightarrow \exists n . n \Vdash x,
$$

so every point $x: M$ has some code $n: N^{\prime}$. The latter belongs only to $x$ since

$$
\begin{aligned}
n \Vdash x \wedge n \Vdash y & \Leftrightarrow A_{n}\{x\} \wedge \beta^{n} x \wedge A_{n}\{y\} \wedge \beta^{n} y \\
& \Rightarrow A_{n}\{x\} \wedge \beta^{n} y \\
& \equiv\left(\exists n . A_{n}\{x\} \wedge \beta^{n}\right) y \\
& \Leftrightarrow\{x\} y \Leftrightarrow\left(x={ }_{M} y\right) .
\end{aligned}
$$

Hence $N^{\prime} \rightarrow \Sigma^{M}$ by $n \mapsto \lambda x . n \Vdash x$ factors through \{\}$: M \longrightarrow \Sigma^{M}$, and $M$ is $N / \sim$ where $(m \sim n) \equiv(\exists x . m \Vdash x \wedge n \Vdash x)[$ C].

We leave it as an exercise to define partial equivalence relations on $\operatorname{List}(N)$ whose quotients are List $(M)$ and $\mathrm{K}(M)$.

Corollary 9.2. Every definable overt discrete object is a subquotient of $\mathbb{N}$ by an open partial equivalence relation.

This does not restrict how "big" overt discrete objects can be in general models of ASD: for example, $\aleph_{1}$ still belongs to the classical model. It simply says that, having required certain base types to be overt discrete, as we did with $\mathbb{N}$ in Axiom 4.4 the additional overt discrete objects that can be constructed from them are no bigger.

Corollary 9.3. In the free model, if an object $X$ has a basis indexed by any overt discrete object $M$ then it has one indexed by $\mathbb{N}$. 
Proof. Let $n: \mathbb{N}, m: M \vdash n \Vdash m$ be the relation defined in the Proposition and $\left(\beta^{m}, A_{m}\right)$ the basis on $X$. Define

$$
\gamma^{n} \equiv \lambda x . \exists m . n \Vdash m \wedge \beta^{m} x \quad \text { and } \quad D_{n} \equiv \lambda \phi . \exists m . n \Vdash m \wedge A_{m} \phi .
$$

So $\gamma^{n}=\beta^{m}$ and $D_{n}=A_{m}$ if $n \Vdash m$, but $\gamma^{n}=\perp$ and $D_{n}=\perp$ if $n \notin N^{\prime}$. Then, using the properties of $\Vdash,\left(\gamma^{n}, D_{n}\right)$ is an effective basis because

$$
\begin{aligned}
\exists n . D_{n} \phi \wedge \gamma^{n} x & \Leftrightarrow \exists n m m^{\prime} . n \Vdash m \wedge A_{m} \phi \wedge n \Vdash m^{\prime} \wedge \beta^{m^{\prime}} x \\
& \Leftrightarrow \exists m . A_{m} \phi \wedge \beta^{m} x \Leftrightarrow \phi x
\end{aligned}
$$

The next goal is Scott continuity, i.e. preservation of directed joins. Recall from Definition 4.13 that these are defined in terms of a structure $(S, 0,+)$ that indexes two families

$$
s: S \vdash \alpha_{s}: \Sigma \text { and } \phi^{s}: \Sigma^{X} .
$$

Lemma 9.4. $\Gamma, \ell: \operatorname{Fin}(M) \vdash\left(\forall m \in \ell . \exists s: S . \alpha_{s} \wedge \phi^{s} m\right) \Leftrightarrow\left(\exists s: S . \alpha_{s} \wedge \forall m \in \ell . \phi^{s} m\right)$.

Proof. We have to show $\Rightarrow$, as $\Leftarrow$ is easy. For the base case, $\ell \equiv 0$, put $s \equiv 0$. For the induction step, $\ell^{\prime}=m:: \ell$, suppose by the induction hypothesis that

$$
\alpha_{t} \wedge \phi^{t} m \wedge \alpha_{s} \wedge \forall m \in \ell . \phi^{s} m
$$

Put $u \equiv s+t: S$, so $\alpha_{u} \Leftrightarrow \alpha_{s+t} \Leftrightarrow \alpha_{s} \wedge \alpha_{t}$ and $\phi^{s}, \phi^{t} \leq \phi^{u}$, so we have $\alpha_{u} \wedge \forall m \in m:: \ell . \phi^{u} m$.

This proof is for lists, but a similar induction scheme works for subsets too, cf. Tay99, Section 6.5]. Since this equational hypothesis is of the form $\sigma \Leftrightarrow \top$, it can be eliminated in favour of an open subobject of the context [E, $\S 2]$.

Lemma 9.5. Any $\Gamma \vdash \mathcal{F}: \Sigma^{3} N$ preserves the directed join $\Gamma \vdash \exists s . \alpha_{s} \wedge \Phi^{s}: \Sigma^{\Sigma^{N}}$.

Proof. Using the previous lemma for $X \equiv \Sigma^{N}$ and $M \equiv \operatorname{Fin} N$,

$$
\begin{array}{rlr}
\mathcal{F}\left(\exists s . \alpha_{s} \wedge \Phi^{s}\right) & \Leftrightarrow \exists L . \mathcal{F} A_{L} \wedge \forall \ell \in L . \exists s . \alpha_{s} \wedge \Phi^{s} \beta^{\ell} & \text { Lemma 8.9] } \\
& \Leftrightarrow \exists L . \mathcal{F} A_{L} \wedge \exists s . \alpha_{s} \wedge \forall \ell \in L . \Phi^{s} \beta^{\ell} & \\
& \Leftrightarrow \exists s . \alpha_{s} \wedge \exists L . \mathcal{F} A_{L} \wedge \forall \ell \in L . \Phi^{s} \beta^{\ell} & \\
& \Leftrightarrow \exists s . \alpha_{s} \wedge \mathcal{F} \Phi^{s} & \text { Lemma 8.9] }
\end{array}
$$

Theorem 9.6. Any $\Gamma \vdash F: \Sigma^{\Sigma^{X}}$ preserves the directed join $\exists s . \alpha_{s} \wedge \phi^{s}: \Sigma^{X}$.

Proof. Making the same substitutions as in Lemma 8.10.

$$
\begin{aligned}
F\left(\lambda x . \exists s . \alpha_{s} \wedge \phi^{s} x\right) & \Leftrightarrow \mathcal{F} \cdot I\left(\lambda x \cdot \exists s \cdot \alpha_{s} \wedge \Phi^{s}(i x)\right) \\
& \Leftrightarrow\left(\mathcal{F} \cdot I \cdot \Sigma^{i}\right)\left(\exists s \cdot \alpha_{s} \wedge \Phi^{s}\right) \\
& \Leftrightarrow \exists s \cdot \alpha_{s} \wedge\left(\mathcal{F} \cdot I \cdot \Sigma^{i}\right) \Phi^{s} \\
& \Leftrightarrow \exists s \cdot \alpha_{s} \wedge F \phi^{s}
\end{aligned}
$$

Corollary 9.7. All $F: \Sigma^{Y} \rightarrow \Sigma^{X}$ preserve directed joins. 
We can now see the construction in Lemma 8.10 as a composite, of Lemma 8.4 twice and the following result. In order to apply $F: \Sigma^{\Sigma^{X}}$ to the basis decomposition of $\phi: \Sigma^{X}$, the decomposition must be a directed join.

Lemma 9.8. If $X$ has a $\vee$-basis then $\Sigma^{X}$ has a prime $\wedge$-basis.

Proof. For $F: \Sigma^{\Sigma^{X}}$ and $\phi: \Sigma^{X}$, since the $\vee$-basis gives a directed join,

$$
F \phi \Leftrightarrow F\left(\exists n . A_{n} \phi \wedge \beta^{n}\right) \Leftrightarrow \exists n . A_{n} \phi \wedge F \beta^{n} .
$$

This is the decomposition of $F$ with respect to the prime $\wedge$-basis with

$$
B^{n} \equiv A_{n} \text { and } \mathcal{A}_{n} \equiv \lambda F . F \beta^{n} .
$$

Notice that it reverses the imposed order on the indexing object.

We can also go up to the second exponential with just one level of lists.

Lemma 9.9. Let $\left(\beta^{n}, A_{n}\right)$ be $a \vee$-basis for $X$. Then

$$
\mathcal{B}^{\ell} \equiv \lambda F . \forall n \in \ell . F \beta^{n} \quad \text { and } \quad \mathbb{A}_{\ell} \equiv \lambda \mathcal{F} . \mathcal{F}\left(\lambda \phi . \exists n \in \ell . A_{n} \phi\right)
$$

define a prime $\wedge$-basis for $\Sigma^{\Sigma^{X}}$, i.e. we have a basis decomposition

$$
\mathcal{F}: \Sigma^{3} X \vdash \mathcal{F}=\lambda F . \exists \ell . \mathcal{F}\left(\lambda \phi . \exists n \in \ell . A_{n} \phi\right) \wedge \forall n \in \ell . F \beta^{n} .
$$

Proof. $\quad\left(A_{n}, \lambda F . F \beta^{n}\right)$ is a basis and $\left(\lambda \phi . \exists n \in \ell . A_{n} \phi, \lambda F . \forall n \in \ell . F \beta^{n}\right)$ an $\vee$-basis for $\Sigma^{X}$ by Lemmas 9.8 and 8.4 . Finally, Lemma 9.8 gives the prime $\wedge$-basis for $\Sigma^{\Sigma^{X}}$.

As a corollary, we have another result similar to Corollary 9.2 although this one does restrict the size of overt discrete compact objects. Such objects are called Kuratowski finite: see $[\mathrm{E}$ ] for more detail. Beware that even finiteness is not the same as being a numeral, for example a Möbius band has a finite set of edges that is locally equal to 2, but this set is not a numeral.

Theorem 9.10. An object $N$ is overt discrete compact iff it is listable, and therefore a quotient of a numeral by an open equivalence relation.

Proof. Expanding the quantifier $\forall_{N}$ using the filter $\vee$-basis for $N$ indexed by List $N$,

$$
\forall_{N} \phi \Leftrightarrow \exists \ell . \forall n . n \in \ell \wedge \forall m \in \ell . \phi m,
$$

so $\top \Leftrightarrow \forall_{N} \top \Leftrightarrow \exists \ell . \forall n . n \in \ell$. The numeral is (the length of) $\ell$, though we require the Existence Property ( $c f$. Remark 4.11) to pin down its order and so make it a quotient of a numeral.

As another corollary, we can complete the business of Proposition 5.11 and Lemma 8.2 .

Theorem 9.11. Let $A: \Sigma^{Y} \rightarrow \Sigma^{X}$ and $B: \Sigma^{V} \rightarrow \Sigma^{U}$.

(a) If $A$ and $B$ both preserve $\top$ and $\wedge$ then the squares

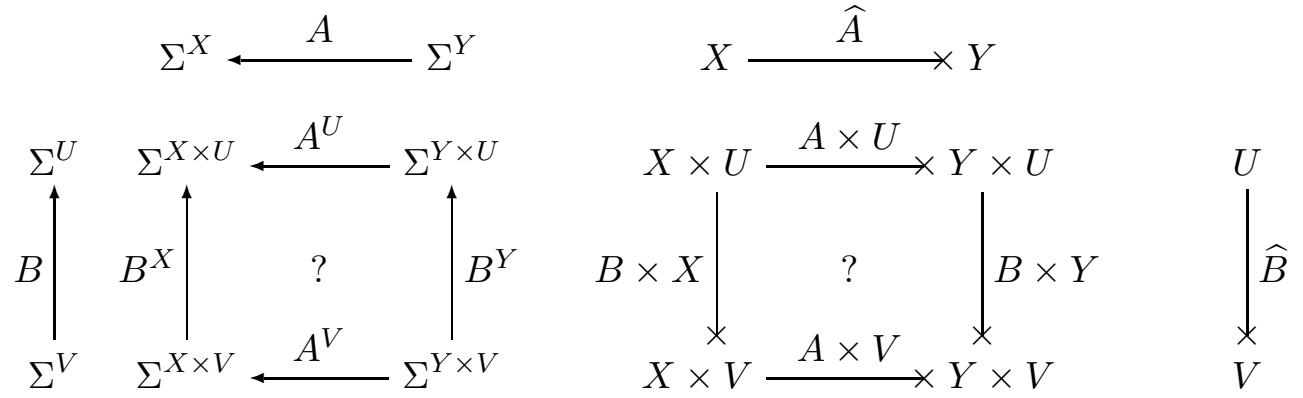


commute. Hence $\times$ is a symmetric monoidal structure in the category whose objects are those of $\mathcal{S}$ and whose morphisms $\widehat{A}: X \rightarrow Y$ are the $A$ that preserve $\top$ and $\wedge$, cf. $\mathrm{A}$, Section 3]. In particular, these maps commute with $\forall_{K}$, for any compact $K$.

(b) If instead $A$ and $B$ both preserve $\perp$ and $\vee$ then again the squares commute, so $\times$ is a symmetric monoidal structure in that category too, and these maps commute with $\exists_{N}$, for any overt $N$.

Proof. Lemma 8.2 and Theorem 9.6 .

Corollary 9.12. Closed and compact subobjects coincide in any compact Hausdorff object, whilst open and overt ones agree in any overt discrete object.

\section{PRIMES AND NUCLEI}

In this section we prove the finitary lattice-theoretic characterisations of primes and nuclei that we announced in Remark 3.4 .

Since homomorphisms of the four lattice connectives also preserve directed joins, we might expect them to be frame homomorphisms. However, they only preserve joins indexed by overt objects, not "arbitrary" ones. On the other hand, objects and inverse image maps are defined in Abstract Stone Duality as algebras and homomorphisms for the monad corresponding to $\Sigma^{(-)} \dashv \Sigma^{(-)}$, rather than for an infinitary algebraic theory.

We actually consider "Curried" homomorphisms.

Definition 10.1. The term $\Gamma \vdash P: \Sigma^{\Sigma^{X}}$ is prime [A, Definition 8.1] if

$$
\Gamma, \mathcal{F}: \Sigma^{3} X \vdash \mathcal{F} P \Leftrightarrow P(\lambda x . \mathcal{F}(\lambda \phi . \phi x)) .
$$

Axiom 3.3(b) says that we may then introduce $a=$ focus $P$ such that $P=\lambda \phi . \phi a$.

Plainly if $P \equiv \lambda \phi . \phi a$ for some $a$ then

$$
P \top \Leftrightarrow \top, \quad P(\phi \wedge \psi) \Leftrightarrow \phi a \wedge \psi a \Leftrightarrow P \phi \wedge P \psi
$$

and similarly for $\perp$ and $\vee$. We know that frame homomorphisms (as defined externally using infinitary lattices) agree with Eilenberg-Moore homomorphisms in the case of the classical models [A, B. Now we can use bases to prove a similar result for the internal finitary lattice structure in our category. This means that we can import at least some of the familiar lattice-theoretic arguments about topology into our category.

Theorem 10.2. $P: \Sigma^{\Sigma^{X}}$ is prime iff it preserves $\top, \perp, \wedge$ and $\vee$.

Proof. $[\Rightarrow]$ Put $\mathcal{F} \equiv \lambda F . F \phi \wedge F \psi$, so $\mathcal{F} P \Leftrightarrow P \phi \wedge P \psi$, whilst

$$
P(\lambda x . \mathcal{F}(\lambda \phi . \phi x)) \Leftrightarrow P(\lambda x . \phi x \wedge \psi x) \Leftrightarrow P(\phi \wedge \psi) .
$$

The other connectives are handled in the same way.

$[\Leftarrow]$ First note that, using Axiom 3.6 for $P$ and $\alpha$, and $P \perp \Leftrightarrow \perp$,

$$
P(\alpha \wedge \phi) \Leftrightarrow P \perp \vee \alpha \wedge P \phi \Leftrightarrow \alpha \wedge P \phi .
$$


Second, $P$ commutes with $\exists$ by Theorem 9.11 (b), so

$$
\begin{array}{rlr}
\mathcal{F} P & \Leftrightarrow \exists \ell . \mathcal{F}\left(\lambda \phi . \exists n \in \ell . A_{n} \phi\right) \wedge \forall n \in \ell . P \beta^{n} & \text { Lemma 9.9 } \\
& \Leftrightarrow \exists \ell . \mathcal{F}\left(\lambda \phi . \exists n \in \ell . A_{n} \phi\right) \wedge P\left(\forall n \in \ell . \beta^{n}\right) & P \text { preserves } \wedge, \top \\
& \Leftrightarrow \exists \ell . P\left(\mathcal{F}\left(\lambda \phi . \exists n \in \ell . A_{n} \phi\right) \wedge \forall n \in \ell . \beta^{n}\right) & \text { above } \\
& \Leftrightarrow P\left(\exists \ell \cdot \mathcal{F}\left(\lambda \phi . \exists n \in \ell . A_{n} \phi\right) \wedge \forall n \in \ell . \beta^{n}\right) & \\
& \Leftrightarrow P\left(\lambda x . \exists \ell \cdot \mathcal{F}\left(\lambda \phi . \exists n \in \ell . A_{n} \phi\right) \wedge \forall n \in \ell .(\lambda \phi . \phi x) \beta^{n}\right) \\
& \Leftrightarrow P(\lambda x . \mathcal{F}(\lambda \phi . \phi x)) . & \text { Lemma 9.9] }
\end{array}
$$

Corollary 10.3. $H: \Sigma^{X} \rightarrow \Sigma^{Y}$ is an Eilenberg-Moore homomorphism iff it is a lattice homomorphism. In this case, it is of the form $H=\Sigma^{f}$ for some unique $f: Y \rightarrow X$.

We have in particular a way of introducing points of an object by finitary latticetheoretic arguments. By this method we can derive a familiar domain-theoretic result, but beware that, as the objects of our category denote locally compact objects and not merely domains, the order by no means characterises the objects and morphisms.

Theorem 10.4. Every object has and every morphism preserves directed joins.

Proof. JJoh82, Lemma II 1.11] Let $\Gamma, s: S \vdash a_{s}: X$ be a directed family in $X$ with respect to the intrinsic order (Definition [3.7), then

$$
\Gamma, s: S \vdash \lambda \phi . \phi a_{s}: \Sigma^{\Sigma^{X}}
$$

is a directed family of primes. But primes are characterised lattice-theoretically, and the property is preserved by directed joins (Theorem 9.6 for $\mathcal{F}$ ), so

$$
\Gamma \vdash P \equiv \lambda \phi . \exists s . \phi a_{s}: \Sigma^{\Sigma^{X}}
$$

is also prime, and $P=\lambda \phi . \phi a$, where $a \equiv$ focus $P: X$.

I claim that this is the join of the given family. By Definition 3.7 the order relation $a_{s} \leq_{X} a$ means $\lambda \phi . \phi a_{s} \leq \lambda \phi . \phi a \equiv P$, which we have by the definition of $P$, whilst similarly if $\Gamma, s: S \vdash a_{s} \leq b$ then $\lambda \phi . \phi a_{s} \leq \lambda \phi$. $\phi b$ so $P \leq \lambda \phi . \phi b$ as $P$ is the join, and then $a \leq b$.

By a similar argument, given $f: X \rightarrow Y$, we have $f\left(a_{s}\right) \leq_{Y} f a$ since $\psi: \Sigma^{Y}$ provides $\psi \cdot f: \Sigma^{X}$, and if $f\left(a_{s}\right) \leq b$ then $f(a) \leq b$, so $f$ preserves the join.

Corollary 10.5. Suppose that an object $X$ has a least element $\perp$ in its intrinsic order. Then any $f: X \rightarrow X$ has a least fixed point, namely

$$
a \equiv \operatorname{focus}\left(\lambda \phi . \exists n . \phi a_{n}\right), \quad \text { where } a_{0} \equiv \perp \text { and } a_{n+1} \equiv f a_{n} .
$$

Finally, we show that the equations in Lemma 7.15 that characterised the Scottcontinuous functions $E$ that are of the form $I \cdot \Sigma^{i}$ for a $\Sigma$-split sublocale are also valid for $\Sigma$-split subobjects in abstract Stone duality.

Definition 10.6. Recall from [B, Definition 4.3] that $E: \Sigma^{X} \rightarrow \Sigma^{X}$ is called a nucleus if

$$
\mathcal{F}: \Sigma^{3} X \vdash E(\lambda x . \mathcal{F}(\lambda \phi . \phi x))=E(\lambda x . \mathcal{F}(\lambda \phi . E \phi x)): \Sigma^{X} .
$$

(This equation arises from Beck's monadicity theorem, and is applicable without assuming any lattice structure on $\Sigma$.) 
Lemma 10.7. If $E$ is a nucleus then

$$
\phi, \psi: \Sigma^{X} \vdash E(\phi \wedge \psi)=E(E \phi \wedge E \psi) \text { and } E(\phi \vee \psi)=E(E \phi \vee E \psi) \text {. }
$$

Proof. Putting $\mathcal{F} \equiv \lambda F . F \phi \wedge F \psi$,

$$
\begin{array}{clrl}
E(\lambda x . \mathcal{F}(\lambda \phi . \phi x))=E(\lambda x . \phi x \wedge \psi x) & =E(\phi \wedge \psi) & & \text { LHS } \\
E(\lambda x . \mathcal{F}(\lambda \phi . E \phi x))=E(\lambda x . E \phi x \wedge E \psi x) & =E(E \phi \wedge E \psi), & & R H S
\end{array}
$$

are equal. The argument for $\vee$ is the same.

For the converse, first observe that the equations allow us to insert or remove $E \mathrm{~s}$ as we please in any sub-term of a lattice expression, so long as $E$ is applied to the whole expression. In particular, $E \phi=E(E \phi)$ and $E(\phi \vee \psi \vee \theta)=E(E \phi \vee E \psi \vee \theta)($ sic $)$.

Lemma 10.8. Although we needn't have $E \top=\top$ or $E \perp=\perp$, we may extend the binary $\checkmark$-formula to finite (possibly empty) sets $\ell: \operatorname{Fin}(N)$ :

$$
E\left(\exists n \in \ell . \alpha_{n} \wedge \phi^{n}\right)=E\left(\exists n \in \ell . \alpha_{n} \wedge E \phi^{n}\right),
$$

where $n: N \vdash \alpha_{n}: \Sigma$ and $\phi^{n}: \Sigma^{X}$. Similarly but more simply, from the $\wedge$-equation,

$$
E\left(\forall n \in \ell . \phi^{n}\right)=E\left(\forall n \in \ell . E \phi^{n}\right) .
$$

Proof. The base case of the induction, $\ell \equiv 0$, is $E \perp=E \perp$, and the next case is

$$
E(\alpha \wedge \phi)=E \perp \vee \alpha \wedge E \phi=E \perp \vee \alpha \wedge E(E \phi)=E(\alpha \wedge E \phi)
$$

by the Phoa principle. For the induction step [E, §2],

$$
\begin{array}{rlr}
E(\exists n & \left.\in m:: \ell . \alpha_{n} \wedge \phi^{n}\right) & \\
& =E\left(\left(\exists n \in \ell . \alpha_{n} \wedge \phi^{n}\right) \vee\left(\alpha_{m} \wedge \phi^{m}\right)\right) & \\
& =E\left(E\left(\exists n \in \ell \cdot \alpha_{n} \wedge \phi^{n}\right) \vee E\left(\alpha_{m} \wedge \phi^{m}\right)\right) & \text { V-equation } \\
& =E\left(E\left(\exists n \in \ell . \alpha_{n} \wedge E \phi^{n}\right) \vee E\left(\alpha_{m} \wedge E \phi^{m}\right)\right) & \text { ind. hyp. } \\
& =E\left(\left(\exists n \in \ell . \alpha_{n} \wedge E \phi^{n}\right) \vee\left(\alpha_{m} \wedge E \phi^{m}\right)\right) & \\
& =E\left(\exists n \in m:: \ell . \alpha_{n} \wedge E \phi^{n}\right) \square
\end{array}
$$

Lemma 10.9. The $\exists$ equation extends by Scott continuity (Proposition 9.6).

Proof.

$$
\begin{aligned}
E\left(\exists n: N . \alpha_{n} \wedge \phi^{n}\right) & =E\left(\exists \ell: \operatorname{Fin} N \cdot \exists n \in \ell \cdot \alpha_{n} \wedge \phi^{n}\right) \\
& =\exists \ell \cdot E\left(\exists n \in \ell \cdot \alpha_{n} \wedge \phi^{n}\right) \\
& =\exists \ell \cdot E\left(\exists n \in \ell \cdot \alpha_{n} \wedge E \phi^{n}\right) \\
& =E\left(\exists n: N \cdot \alpha_{n} \wedge E \phi^{n}\right)
\end{aligned}
$$

similarly

Theorem 10.10. $E$ is a nucleus iff it satisfies

$$
\phi, \psi: \Sigma^{X} \vdash E(\phi \wedge \psi)=E(E \phi \wedge E \psi) \text { and } E(\phi \vee \psi)=E(E \phi \vee E \psi) \text {. }
$$

Proof. We expand $\mathcal{F}: \Sigma^{3} X$ in the defining equation for a nucleus

$$
\begin{array}{rlr}
E(\lambda x . \mathcal{F}(\lambda \phi . E \phi x)) & =E\left(\exists L . \mathcal{F} A_{L} \wedge \forall \ell \in L . E \beta^{\ell}\right) & \text { Proposition 9.8 } \\
& =E\left(\exists L . \mathcal{F} A_{L} \wedge E\left(\forall \ell \in L . E \beta^{\ell}\right)\right) & \text { Lemma 10.9 } \\
& =E\left(\exists L . \mathcal{F} A_{L} \wedge E\left(\forall \ell \in L . \beta^{\ell}\right)\right) & \text { Lemma 10.8 } \\
& =E\left(\exists L . \mathcal{F} A_{L} \wedge \forall \ell \in L . \beta^{\ell}\right) & \text { Lemma 10.9 } \\
& =E(\lambda x . \mathcal{F}(\lambda \phi . \phi x)) & \text { Proposition } 9.8 \square
\end{array}
$$




\section{The WAY-Below RELATion}

We now introduce the last (g) of our abstract characterisations of local compactness in the Introduction. Recall from Section 2 that any continuous distributive lattice carries a binary relation (written $\ll$ and called "way-below") such that

$$
\begin{aligned}
& \perp \ll \gamma \quad \frac{\alpha \ll \gamma \beta \ll \gamma}{\alpha \vee \beta \ll \gamma} \quad \frac{\alpha^{\prime} \leq \alpha \ll \beta \leq \beta^{\prime}}{\alpha^{\prime} \ll \beta^{\prime}} \quad \frac{\alpha \ll \gamma}{\overline{\exists \beta . \alpha \ll \beta \ll \gamma}} \\
& \frac{\alpha \ll \beta \quad \beta \ll \phi \quad \beta \ll \psi}{\alpha \ll(\phi \wedge \psi)} \quad \frac{\alpha \ll \beta \vee \gamma}{\overline{\exists \beta^{\prime} \gamma^{\prime} . \alpha \ll \beta^{\prime} \vee \gamma^{\prime} \beta^{\prime} \ll \beta \gamma^{\prime} \ll \gamma}}
\end{aligned}
$$

Notation 11.1. We define a new relation $n \preccurlyeq m$ as $A_{n} \beta^{m}$.

This is an open binary relation (term of type $\Sigma$ with two free variables) on the overt discrete object $N$ of indices of a basis, not on the lattice $\Sigma^{X}$. It is an "imposed" structure on $N$ in the sense of Remark 4.5 .

For most of the results of this section, we shall require $\left(\beta^{n}, A_{n}\right)$ to be an $\vee$-basis for $X$.

Examples 11.2. (Not all of these are $\vee$-bases.)

(a) Let $\beta^{n}$ classify $U^{n} \subset X$, and $A_{n}=\lambda \phi$. $\left(K^{n} \subset \phi\right)$ in a locally compact sober space. Then $n \nVdash m$ means that $K^{n} \subset U^{m}$. This is consistent with Notation 1.3 if we identify the basis element $n$ with the pair $\left(U^{n} \subset K^{n}\right)$.

(b) Let $A_{n}=\lambda \phi .\left(\beta^{n} \ll \phi\right)$ in a continuous lattice. Then $n \preccurlyeq m$ means that $\beta^{n} \ll \beta^{m}$, cf. Definition 2.3.

(c) In the interval basis on $\mathbb{R}$ in Example 6.10, $\langle q, \delta\rangle \prec\langle p, \epsilon\rangle$ means that $[q, \delta] \subset(p, \epsilon)$, i.e. $p-\epsilon<q-\delta \leq q+\delta<p+\epsilon$.

(d) In the basis of disjoint pairs of opens, $\left(U^{n} \pitchfork V_{n}\right)$, for a compact Hausdorff space in Example 6.11 $n \preccurlyeq m$ means that $V_{n} \cup U^{m}=X$.

(e) In the prime basis $(\{n\}, \eta n)$ for $N$ in Example 6.7(a) $n \preccurlyeq m$ just when $n=m$.

(f) In the Fin $(N)$-indexed filter $\vee$-basis on $N$ in Proposition 8.8 , $\ell \ll \ell^{\prime}$ iff $\ell \subset \ell^{\prime}$.

(g) In the prime $\wedge$-basis on $\Sigma^{N}$ in Example 6.7(b) on the other hand, $\ell \preccurlyeq \ell^{\prime}$ iff $\ell^{\prime} \subset \ell$.

(h) More generally, in the prime $\wedge$-basis on $\Sigma^{X}$ derived from an $\vee$-basis on $X$ in Lemma 9.8 , $\left(n \gtrless_{\Sigma X} m\right)$ iff $\left(m \preccurlyeq_{X} n\right)$.

(i) In the Fin $(\operatorname{Fin}(N))$-indexed filter lattice basis on $\Sigma^{N}$ in Proposition 8.8,

$$
L \prec R \equiv \mathcal{A}_{L} B^{R} \Leftrightarrow R \subset^{\sharp} L \equiv \forall \ell \in L . \exists \ell^{\prime} \in R .\left(\ell^{\prime} \subset \ell\right),
$$

where $R \subset^{\sharp} L$ is known as the upper order on subsets induced by the relation $\subset$ on elements.

(j) In the prime $\wedge$-basis on $\Sigma^{\Sigma^{N}}$ in Lemma 8.9 $L \nVdash R$ iff $L \subset^{\sharp} R$.

(k) In the $\vee$-basis on $\Sigma^{\Sigma^{X}}$ derived from an $\vee$-basis on $X$ in Lemma 9.9.

$$
\left(\ell \aleph_{\Sigma^{2} X} \ell^{\prime}\right) \Longleftrightarrow\left(\ell \varkappa^{\sharp} \ell^{\prime}\right) \text {. }
$$

(l) Any stably locally compact object $X$ has a filter lattice basis $(N, 0,1,+, \star, \preccurlyeq)$ such that the opposite $(N, 1,0, \star,+, \nsucc)$ is the basis of another such object, known as its Lawson dual (Proposition 12.14).

Our first result just restates the assumption of an $\vee$-basis, $c f$. Lemmas 1.9 and 2.6

Lemma 11.3. $0 \preccurlyeq p$, whilst $n+m \preccurlyeq p$ iff both $n \preccurlyeq p$ and $m \nVdash p$.

Proof. $\quad A_{0} \beta^{p} \Leftrightarrow \top$ and $A_{n+m} \beta^{p} \Leftrightarrow A_{n} \beta^{p} \wedge A_{m} \beta^{p}$. 
In a continuous lattice, $\alpha \ll \beta$ implies $\alpha \leq \beta$, but we have no similar property relating $\preccurlyeq$ to $\preccurlyeq$. We shall see the reasons for this in Section [13, But we do have two properties that carry most of the force of $\alpha \ll \beta \Rightarrow \alpha \leq \beta$. We call them roundedness. The second also incorporates many of the uses of directed joins and Scott continuity into a notation that will become increasingly more like discrete mathematics than it resembles the technology of traditional topology.

Lemma 11.4. $\beta^{n}=\exists m$. $(m \prec n) \wedge \beta^{m}$ and $A_{m}=\exists n$. $A_{n} \wedge(m \prec n)$.

Proof. The first is simply the basis expansion of $\beta^{n}$. For the second, we apply $A_{m}$ to the basis expansion of $\phi$, so

$$
A_{m} \phi \Leftrightarrow \exists n . A_{n} \phi \wedge A_{m} \beta^{n} \Leftrightarrow\left(\exists n . A_{n} \wedge A_{m} \beta^{n}\right) \phi,
$$

since $A_{n}$ preserves directed joins (Theorem 9.6).

Corollary 11.5. If $m \nVdash n$ then $\beta^{m} \leq \beta^{n}$ and $A_{m} \geq A_{n}$.

Corollary 11.6. If $m \ll n, A_{m^{\prime}} \geq A_{m}$ and $\beta^{n} \leq \beta^{n^{\prime}}$, then $m^{\prime} \preccurlyeq n^{\prime}$.

Proof. $\quad(m \ll n) \equiv A_{m} \beta^{n} \Rightarrow A_{m^{\prime}} \beta^{n^{\prime}} \equiv\left(m^{\prime} \ll n^{\prime}\right)$.

Corollary 11.7. The relation $\nleftarrow$ satisfies transitivity and the interpolation lemma:

$$
(m \prec n) \Leftrightarrow(\exists k . m \prec k \preccurlyeq n) .
$$

Proof. $\quad A_{m} \beta^{n} \Leftrightarrow\left(\exists k . A_{k} \wedge m \prec k\right) \beta^{n} \Leftrightarrow \exists k . A_{k} \beta^{n} \wedge m \prec k$.

Now we consider the interaction between $\prec$ and the lattice structures $(\top, \perp, \wedge, \vee)$ and $(1,0,+, \star)$. Of course, for this we need a lattice basis.

Lemma 11.8. As directed joins,

$$
\phi \wedge \psi=\exists p q . \beta^{p \star q} \wedge A_{p} \phi \wedge A_{q} \psi \quad \text { and } \quad \phi \vee \psi=\exists p q . \beta^{p+q} \wedge A_{p} \phi \wedge A_{q} \psi
$$

Proof. The first is the Frobenius law, since $\beta^{p \star q}=\beta^{p} \wedge \beta^{q}$.

The second uses Lemma 4.17 we obtain the expression

$$
\phi \vee \psi=\exists p . A_{p} \phi \wedge\left(\beta^{p} \vee \psi\right)
$$

from the basis expansion $\phi=\exists p . A_{p} \phi \wedge \beta^{p}$ by adding $\psi$ to the 0 th term (since $A_{0} \phi \Leftrightarrow \top$ and $\beta^{0}=\perp$ ) and, harmlessly, $A_{p} \phi \wedge \psi$ to the other terms. Similarly,

$$
\beta^{p} \vee \psi=\exists q . A_{q} \psi \wedge\left(\beta^{p} \vee \beta^{q}\right)=\exists q . A_{q} \psi \wedge \beta^{p+q} .
$$

The joins are directed because because $A_{0} \phi \wedge A_{0} \psi \Leftrightarrow \top$ and

$$
\left(A_{p_{1}} \phi \wedge A_{q_{1}} \psi\right) \wedge\left(A_{p_{2}} \phi \wedge A_{q_{2}} \psi\right) \Leftrightarrow\left(A_{p_{1}+p_{2}} \phi \wedge A_{q_{1}+q_{2}} \psi\right) .
$$

Lemma 11.9. For a lattice basis, $A_{n} \top \Leftrightarrow(n \prec 1), A_{n} \perp \Leftrightarrow(n \prec 0)$ and

$$
\begin{aligned}
& A_{n}(\phi \wedge \psi) \Leftrightarrow \exists p q .(n \preccurlyeq p \star q) \wedge A_{p} \phi \wedge A_{q} \psi \\
& A_{n}(\phi \vee \psi) \Leftrightarrow \exists p q .(n \preccurlyeq p+q) \wedge A_{p} \phi \wedge A_{q} \psi
\end{aligned}
$$

Proof. The first two are $A_{n} \beta^{1}$ and $A_{n} \beta^{0}$. The other two are $\exists p q . A_{n}\left(\beta^{p \star q}\right) \wedge A_{p} \phi \wedge A_{q} \psi$ and $\exists p q . A_{n}\left(\beta^{p+q}\right) \wedge A_{p} \phi \wedge A_{q} \psi$, which are $A_{n}$ applied to the directed joins in Lemma 11.8, 
Proposition 11.10. The lattice basis $\left(\beta^{n}, A_{n}\right)$ is a filter basis iff $1 \prec 1$ and

$$
\frac{m \preccurlyeq p \star q}{\overline{m \ll p \quad m \ll q}}
$$

Proof. $\quad(n \nVdash 1) \Leftrightarrow A_{n} \beta^{1} \Leftrightarrow A_{n} \top$, but recall that $n \nVdash 1$ for all $n$ iff $1 \prec 1$.

The displayed rule is $A_{m} \beta^{p} \wedge A_{m} \beta^{q} \Leftrightarrow A_{m} \beta^{p \star q} \equiv A_{m}\left(\beta^{p} \wedge \beta^{q}\right)$. Given this, by the Frobenius law and Lemma 11.9

$$
\begin{aligned}
A_{m} \phi \wedge A_{m} \psi & \Leftrightarrow \exists p q . A_{p} \phi \wedge A_{q} \psi \wedge A_{m} \beta^{p} \wedge A_{m} \beta^{q} \\
& \Leftrightarrow \exists p q . A_{p} \phi \wedge A_{q} \psi \wedge(m \preccurlyeq p) \wedge(m \preccurlyeq q) \\
& \Leftrightarrow \exists p q . A_{p} \phi \wedge A_{q} \psi \wedge(m \preccurlyeq p \star q) \\
& \Leftrightarrow A_{m}(\phi \wedge \psi)
\end{aligned}
$$

If we don't have a filter basis, we have to let $n$ "slip" by $n \preccurlyeq m$, cf. Lemma 2.8

Lemma 11.11. For any lattice basis,

$$
\frac{n \preccurlyeq p \star q}{\overline{\exists m .(n \prec m) \wedge(m \prec p) \wedge(m \prec q)}}
$$

Proof. Downwards, interpolate $n \preccurlyeq m \preccurlyeq p \star q \preccurlyeq p, q$, then $m \preccurlyeq p, q$ by monotonicity. Conversely, using Corollary [11.5] if $\mathcal{A}_{n} \beta^{m} \Leftrightarrow \top$ and $\beta^{m} \leq \beta^{p \star q}$ then $A_{n} \beta^{p \star q} \Leftrightarrow \top$.

The corresponding result for $\vee$ is our version of the Wilker property, $c f$. Proposition 1.10 and Lemma 2.7

Lemma 11.12.

$$
\frac{n \preccurlyeq p+q}{\exists p^{\prime} q^{\prime} \cdot\left(n \preccurlyeq p^{\prime}+q^{\prime}\right) \wedge\left(p^{\prime} \preccurlyeq p\right) \wedge\left(q^{\prime} \preccurlyeq q\right)}
$$

Proof. Lemma 11.9 with $\phi \equiv \beta^{p}$ and $\psi \equiv \beta^{q}$.

We shall summarise these rules in Definition 14.1

There are special results that we have in the cases of overt and compact objects. We already know that $1 \preccurlyeq 1$ iff the object is compact ( $c f$. Lemma 6.5), but the lattice dual characterisation of overtness cannot be $0 \preccurlyeq 0$, as that always happens.

Lemma 11.13. If $X$ is overt then

$$
(n \preccurlyeq m) \Rightarrow(n \preccurlyeq 0) \vee\left(\exists y \cdot \beta^{m} y\right) \quad \text { but } \quad(n \preccurlyeq 0) \wedge\left(\exists x . \beta^{n} x\right) \Leftrightarrow \perp \text {. }
$$

Proof. $\quad \phi x \Rightarrow \exists y . \phi y$ so, using the Phoa principle (Axiom [3.6),

$$
A_{n} \phi \Rightarrow A_{n}(\lambda x . \exists y . \phi y) \Leftrightarrow A_{n} \perp \vee \exists y . \phi y \wedge A_{n} \top \Rightarrow(n \prec 0) \vee \exists y . \phi y .
$$

Putting $\phi \equiv \beta^{m}, \quad(n \preccurlyeq m) \equiv A_{n} \beta^{m} \Rightarrow(n \preccurlyeq 0) \vee \exists y \cdot \beta^{m} y$, whilst $(n \ll 0) \wedge \exists x . \beta^{n} x \Leftrightarrow \exists x . A_{n} \beta^{0} \wedge \beta^{n} x \Rightarrow \exists x . \exists n . A_{n} \perp \wedge \beta^{n} x \Leftrightarrow \exists x . \perp x \Leftrightarrow \perp$.

By a similar argument, we have Johnstone's "Townsend-Thoresen Lemma" [Joh84],

$$
(n \preccurlyeq p+q) \Rightarrow(n \preccurlyeq p) \vee\left(\exists y \cdot \beta^{q} y\right) \text {. }
$$

Corollary 11.14. Given a compact overt object, it's decidable whether it's empty or inhabited, cf. Corollary 5.8.

Proof. As $(1 \prec 1) \Leftrightarrow \top$, the Lemma makes $(1 \prec 0)$ and $\exists x . \beta^{1} x \equiv \exists x$. $\top$ complementary. 
Compact overt subobjects are, in fact, particularly well behaved in discrete $[\mathrm{E}]$ and Hausdorff [J] objects.

Sections [13 16] are devoted to proving that the rules above for $\preccurlyeq$ are complete, in the sense that from any abstract basis $(N, 0,1,+, \star, \gtrless)$ satisfying them we may recover an object of the category. This proof is very technical, so in the next section we consider a special case, in which the lattice structure plays a much lighter role.

\section{Domain THEORY IN ASD}

In this section we provide the basic tools for implementing domain theory within ASD. Denotational semantics can then be developed in the usual way using this, allowing programming languages such as Gordon Plotkin's PCF Plo77 to be interpreted in ASD, and with them the programs required in Definitions [1.5, [1.6] and 2.3 for computable bases and functions. The difference is that classical domain theory leads to the dead end of set theory, whilst ASD can be translated back into programming languages.

However, the denotational semantics of programming languages will have to wait for a separate investigation. In this section we merely provide a few results that connect ASD with the topological aspects of domain theory such as [JKM99, JKM01, JS96, that rely on manipulation of bases (in the sense of that subject).

We first characterise the objects that have prime bases in our sense, and are continuous dcpos in the classical setting. The following results are essentially those of [Smy77, §2], where $\triangleleft$ is called an R-structure. However, whereas Michael Smyth imposed a separate theory of recursion on the existing set-based theory of continuous dcpos, recursion is intrinsic to ASD. We also form the domain of rounded ideals using our own technology, which was developed in $\mathrm{B}$.

Definition 12.1. A directed interpolative relation $n, m: N \vdash(n \triangleleft m): \Sigma$ on an overt discrete object $N$ is an open relation that satisfies:

(a) transitivity and interpolation: $n, m: N \vdash(n \triangleleft m) \Leftrightarrow \exists k$. $(n \triangleleft k) \wedge(k \triangleleft m)$;

(b) extrapolation downwards: $n: N \vdash \exists k .(k \triangleleft n) \Leftrightarrow \top$;

(c) directedness: $n, r, s: N \vdash(r \triangleleft n) \wedge(s \triangleleft n) \Leftrightarrow \exists k$. $(r \triangleleft k) \wedge(s \triangleleft k) \wedge(k \triangleleft n)$.

A rounded ideal for $(N, \triangleleft)$ is a predicate $\Gamma \vdash \xi: \Sigma^{N}$ that is

(a) rounded: $\Gamma, n: N \vdash \xi n \Leftrightarrow \exists k .(n \triangleleft k) \wedge \xi k$;

(b) inhabited: $\Gamma \vdash \exists k . \xi k \Leftrightarrow \top$;

(c) an ideal: $\Gamma, r, s: N \vdash \xi r \wedge \xi s \Rightarrow \exists k .(r \triangleleft k) \wedge(s \triangleleft k) \wedge \xi k$.

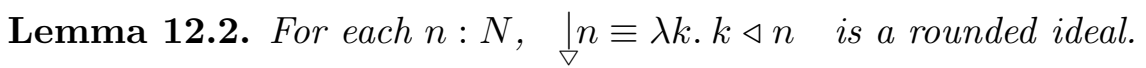

We shall see that the $\underset{\nabla}{\mid}$ provide the "basic points" $p_{n}$ (cf. Definition 1.5) of continuous and algebraic dcpos. In fact, the situation is summed up by the next result, whose converse we shall prove in Theorem 12.11

Proposition 12.3. If $X$ has an $N$-indexed prime basis, so

$$
\phi x \Leftrightarrow \exists n . \phi p_{n} \wedge \beta^{n} x
$$

then $\succcurlyeq$ is a directed interpolative relation, and $x: X \vdash \xi \equiv \lambda n . \beta^{n} x$ is a rounded ideal, with

$$
x=\bigvee\left\{p_{n} \mid \xi n\right\} \text {. }
$$


Beware that $\preccurlyeq$ and $\triangleleft$ have opposite senses, following the conventions for continuous lattices, $c f$. the use of $\ll$ for compact and open subspaces in Notation 1.3. we think of $\triangleleft$ as relating points. Thus $m \succcurlyeq n$ and $m \triangleleft n$ mean that $A_{m} \ll A_{n}$ and $p_{m} \ll p_{n}$, but $\beta^{n} \ll \beta^{m}$.

Proof. We have already proved these properties when $\nVdash$ arises from a filter $\vee$-basis. An $\vee$-basis was needed in order to use Scott continuity for $A_{n}$, but in this case $A_{n} \equiv \lambda \phi . \phi p_{n}$ preserves finite joins too, so directedness is redundant. Briefly,

(a) the basis expansion of $(n \preccurlyeq m) \equiv \beta^{m} p_{n}$ gives transitivity and interpolation, as in Corollary 11.7

(b) that of $(\lambda x . \top) p_{n}$ gives extrapolation; whilst

(c) that of $\left(\beta^{r} \wedge \beta^{s}\right) p_{n}$ gives directedness, as in Proposition 11.10 .

The directed join is focus $(\lambda \phi .-)$ applied to the basis expansion, as in Theorem 10.4

Examples 12.4. Here again are the prime bases that we have encountered.

(a) Equality or any open equivalence relation on an overt discrete object is a directed interpolative relation, whose rounded ideals are the equivalence classes.

(b) $\Sigma^{N}$ is the object of (rounded) ideals for list or subset inclusion in $\operatorname{Fin}(N)$.

(c) If $X$ has an $N$-indexed $\vee$-basis, $\prec_{X}$ is a directed interpolative relation on $N$, and $\Sigma^{X}$ is the object of rounded ideals for this (recall from Lemma 9.8 that $\gtrless_{\Sigma X}$ is $\succcurlyeq_{X}$ ).

(d) In the case of an $N$-indexed filter lattice basis (so $X$ is stably locally compact), the opposite relation $\left(\succcurlyeq_{X}\right)$ is also directed interpolative, because

$\exists k . A_{n} \beta^{k} \Leftrightarrow A_{n} \beta^{1} \Leftrightarrow A_{n}^{\top} \Leftrightarrow \top \quad A_{n} \beta^{r} \wedge A_{n} \beta^{s} \Leftrightarrow A_{n}\left(\beta^{r} \wedge \beta^{s}\right) \Leftrightarrow A_{n}\left(\beta^{r+s}\right)$.

(e) Classically, the rounded ideals for $\succcurlyeq$ are in order-reversing bijection with the compact saturated subspaces of $X$ (Theorem 5.18).

Lemma 12.5. Any reflexive transitive relation is directed interpolative, and then all ideals are rounded.

Examples 12.6. (a) If the way-below relation $\preccurlyeq$ of the space $X$ in Proposition 12.3 is reflexive then $X$ is called an algebraic dcpo, and the $p_{n}$ are its so-called finite or compact elements.

(b) The space $X$ is a continuous lattice iff it has a basis $(N, \triangleleft)$ that's a semilattice, i.e. the extrapolative and directed conditions are strengthened to a least element and binary joins respectively, instead of the extra/interpolant $k$.

(c) In a Scott domain, the join is conditional: the join $r+s$ exists in $N$ whenever there is some upper bound $n$. Any Scott domain is a closed subspace of a continuous lattice, but if they are to form a cartesian closed category, they must also be overt $[\mathrm{F}]$. Indeed, the basis $N$ is overt in the formulation of this section. Instead of a conditional join, we may add a formal top element to $N$, or, equivalently, specify what part of an enlarged basis is to define the required domain by means of a decidable consistency predicate, cf. Proposition 7.12 Sco82.

(d) With pushouts (joins of pairs that are bounded below) but not necessarily a least element, we have a class of objects that includes the overt discrete ones and is closed under $\Sigma^{(-)}$, sums and products. The structure of this subcategory is simple enough to be axiomatised directly in terms of overt discrete objects, i.e. from an arithmetic 
universe $\mathrm{E}$. On the other hand, the whole category $\mathcal{S}$ may be recovered from it by the monadic construction in $[\mathrm{B}]$.

(e) In an $\boldsymbol{L}$-domain, the conditional joins in (c) are replaced by joins within the lower subset $\underset{\nabla}{\mid} n$.

(f) If you are familiar with the characterisation of SFP or bifinite domains, and their continuous generalisations, you will be able to see how to encode them and their bases in ASD. Maybe this could provide a way of making the results about them more constructive, and identifying the largest cartesian closed category of locally compact objects Jun90.

In the classical theory, a domain is recovered from its basis as the (continuous or algebraic) dcpo of (rounded) ideals, with the Scott topology. We can prove the corresponding result within ASD.

Lemma 12.7. For any directed interpolative relation $(N, \triangleleft)$,

$$
\Phi: \Sigma^{\Sigma^{N}}, \xi: \Sigma^{N} \vdash \mathcal{E} \Phi \xi \equiv \exists n . \Phi\left(\left.\right|_{\nabla} n\right) \wedge \xi n
$$

is a nucleus on $\Sigma^{N}$.

Proof. We have a directed ${ }^{6}$ union

$$
(\mid n) \Leftrightarrow \lambda m . \exists k .(m \triangleleft k \triangleleft n) \Leftrightarrow \exists k .(\mid k) \wedge(k \triangleleft n)
$$

by the extrapolation and directedness conditions, so by Theorem 9.6 and the definition of $\mathcal{E}$,

$$
\Phi(\mid n) \Leftrightarrow \exists k . \Phi(\mid k) \wedge(k \triangleleft n) \Leftrightarrow \mathcal{E} \Phi(\mid n) .
$$

Hence we deduce the equations for a nucleus in Theorem 10.10

$$
\begin{aligned}
\mathcal{E}(\Phi \odot \Psi) \xi & \equiv \exists n .(\Phi(\mid n) \odot \Psi(\mid n)) \wedge \xi n \\
& \equiv \exists n .(\mathcal{E} \Phi(\mid n) \odot \mathcal{E} \Psi(\mid n)) \wedge \xi n \\
& \equiv \mathcal{E}(\mathcal{E} \Phi \odot \mathcal{E} \Psi) \xi .
\end{aligned}
$$

Definition 12.8. We have defined $\mathcal{E}$ from entirely abstract data (a directed interpolative relation) but have shown (via Theorem 10.10) that it satisfies the characteristic property of the composite $\Sigma^{i} \cdot I$ that arises from a $\Sigma$-split subobject.

Now, it was exactly the purpose of the monadic calculus [B, $\S 8]$ that, in this situation, we do have an object, called $X \equiv\left\{\Sigma^{N} \mid \mathcal{E}\right\}$ there, that has such a $\Sigma$-split inclusion. That is, the calculus provides $i:\left\{\Sigma^{N} \mid \mathcal{E}\right\} \longmapsto \Sigma^{N}$ and $I$ formally. Then $\Gamma \vdash \xi: \Sigma^{N}$ is admissible, i.e. an element of the new subobject, if

$$
\Gamma, \Phi: \Sigma^{\Sigma^{N}} \vdash \mathcal{E} \Phi \xi \Leftrightarrow \Phi \xi .
$$

The idea of this is that $\mathcal{E}$ "normalises" open subobjects $\Phi$ of $\Sigma^{N}$ by restricting and reexpanding them, and it is only the elements of the subobject being defined whose membership of all such $\Phi$ remains unchanged in this process.

In this case, the calculus allows us to introduce $x \equiv \operatorname{admit} \xi: X$ such that $\xi=i x$.

\footnotetext{
${ }^{6}$ It is directed in the existential sense of Definition 2.1(a) not in the canonical one of Definition 4.13 needed for Theorem 9.6 An $\mathbb{N}-\mathbb{N}$ choice principle is needed for open (recursively enumerable) relations that amounts to sequentialising non-deterministic programs. This will be justified and applied to other questions arising from this paper in future work $\mathrm{G}-$.
} 
Lemma 12.9. $\xi: \Sigma^{N}$ is admissible iff it is a rounded ideal.

Proof. Putting $\Phi \equiv \lambda \theta . \theta n, \Phi \equiv \lambda \theta . \top$ and $\Phi \equiv \lambda \theta . \theta r \wedge \theta s$, we deduce that $\xi$ is a rounded ideal. Conversely, if $\xi$ is a rounded ideal then

$$
\forall m \in \ell . \xi m \Leftrightarrow \exists k .(\forall m \in \ell . m \triangleleft k) \wedge \xi k
$$

by equational induction on $\ell$. Then, using the prime $\wedge$-basis on $\Sigma^{N}$,

$$
\begin{aligned}
\Phi \xi & \Leftrightarrow \quad \exists . \Phi(\lambda m . m \in \ell) \wedge \forall m \in \ell . \xi m \\
\Phi(\mid k) & \Leftrightarrow \quad \exists l \Phi(\lambda m . m \in \ell) \wedge \forall m \in \ell . m \triangleleft k \\
\Phi \xi & \Leftrightarrow \quad \exists \ell . \Phi(\lambda m . m \in \ell) \wedge \exists k .(\forall m \in \ell . m \triangleleft k) \wedge \xi k \\
& \Leftrightarrow \quad \exists k . \Phi(\mid k) \wedge \xi k \equiv \mathcal{E} \Phi \xi .
\end{aligned}
$$

Corollary 12.10. $\left.\right|_{\nabla} n$ is admissible, because it is a rounded ideal.

Theorem 12.11. Any directed interpolative relation $(N, \triangleleft)$ is the opposite of the way-below relation for a prime basis on its object of rounded ideals.

Proof. Let $X \equiv\left\{\Sigma^{N} \mid \mathcal{E}\right\} \stackrel{i}{\longrightarrow} \Sigma^{N}$ in the notation of [B, Section 8]. Now I claim that

$$
p_{n} \equiv \operatorname{admit}(\mid n) \text { and } \beta^{n} \equiv \lambda x . i x n \equiv \Sigma^{i}(\lambda \xi . \xi n)
$$

define a prime basis. Since $\xi \equiv i x$ is admissible, we have the basis expansion,

$$
\begin{aligned}
\phi x \Leftrightarrow I \phi(i x) \Leftrightarrow \mathcal{E}(I \phi)(i x) & \Leftrightarrow \exists n . I \phi(\mid n) \wedge i x n \\
& \Leftrightarrow \exists n . I \phi\left(i p_{k}\right) \wedge \beta^{n} x \\
& \Leftrightarrow \exists n . \phi p_{k} \wedge \beta^{n} x .
\end{aligned}
$$

Finally, $(n \preccurlyeq m) \Leftrightarrow A_{n} \beta^{m} \Leftrightarrow \beta^{m} p_{n} \Leftrightarrow i(\operatorname{admit}(\mid n)) m \Leftrightarrow(\mid n) m \Leftrightarrow(m \triangleleft n)$.

We leave domain theory aside there and return to topology.

Remark 12.12. Jung and Sünderhauf characterise stably locally compact spaces using a system of rules that they call a strong proximity lattice [JS96, Section 5]. These axioms are very similar to those in Section [1] except that theirs are lattice dual, whereas ours are not. In particular, they prove the dual Wilker property (Corollary [5.19), albeit using Choice. If $(N, 0,1,+, \star, \nVdash)$ is an abstract basis satisfying these axioms then so too is $(N, 1,0, \star,+, \nsucc)$. The corresponding object, which is known as the Lawson dual, classically has the same points as the given one, but the opposite specialisation order, whilst the open subobjects of one correspond to the compact saturated subobjects of the other.

Example 12.13. Let $\preccurlyeq$ be the way-below relation for a filter lattice basis on a stably locally compact space $X$. The object $\mathcal{P}^{\sharp} X$ of rounded ideals for $\succcurlyeq$ (Example 12.4(d)] is called the Smyth or upper powerdomain of $X$ and is Lawson dual to the topology $\Sigma^{X}$. 
Lawson duality goes way beyond the purposes of this paper, but we can achieve the dual Wilker property by defining a new basis.

Proposition 12.14. Any stably locally compact object, i.e. one that has a filter $\wedge$-basis $(N, 0,1,+, \star, \preccurlyeq)$, has another such basis indexed by $\operatorname{Fin}(N)$ that also satisfies the dual Wilker property,

$$
\frac{p \star q \preccurlyeq n}{\overline{\exists p^{\prime} q^{\prime} \cdot\left(p^{\prime} \star q^{\prime} \preccurlyeq n\right) \wedge\left(p \preccurlyeq p^{\prime}\right) \wedge\left(q \prec q^{\prime}\right)} .}
$$

This can be extended to a new filter lattice basis with the same property by Lemma 8.4ff.

Proof. We define a new version of $\star$, called $\times$, by

$$
A_{p \times q} \equiv \exists p^{\prime} q^{\prime} . A_{p^{\prime} \star q^{\prime}} \wedge\left(p \preccurlyeq p^{\prime}\right) \wedge\left(q \prec q^{\prime}\right) .
$$

This construction, unlike Remark 8.7. preserves the filter property, and is also idempotent.

$$
\begin{aligned}
& A_{p \times q} \top \Leftrightarrow \exists p^{\prime} q^{\prime} . A_{p^{\prime} \star q^{\prime}} \top \wedge\left(p \prec p^{\prime}\right) \wedge\left(q \prec q^{\prime}\right) \\
& \Leftarrow(p \prec 1) \wedge(q \prec 1) \Leftrightarrow \top \\
& A_{p \times q}=\exists p^{\prime \prime} q^{\prime \prime} \cdot A_{p^{\prime \prime} \nless q^{\prime \prime}} \wedge\left(p \preccurlyeq p^{\prime \prime}\right) \wedge\left(q \preccurlyeq q^{\prime \prime}\right) \quad \operatorname{def} \times \\
& =\exists p^{\prime} q^{\prime} p^{\prime \prime} q^{\prime \prime} . A_{p^{\prime \prime} \star q^{\prime \prime}} \wedge\left(p \prec p^{\prime} \preccurlyeq p^{\prime \prime}\right) \wedge\left(q \prec q^{\prime} \preccurlyeq q^{\prime \prime}\right) \text { interpolation } \\
& =\exists p^{\prime} q^{\prime} \cdot A_{p^{\prime} \times q^{\prime}} \wedge\left(p \preccurlyeq p^{\prime}\right) \wedge\left(q \prec q^{\prime}\right) \quad \operatorname{def} \times
\end{aligned}
$$

We deduce the dual Wilker property by applying the last equation to $\beta^{n}$. Now, as the $A_{n}$ preserve $\wedge$,

$$
\begin{aligned}
& A_{p \times q}(\phi \wedge \psi) \quad \Leftrightarrow \quad \exists p^{\prime \prime \prime} q^{\prime \prime \prime} . A_{p^{\prime \prime \prime} \nless q^{\prime \prime \prime}}(\phi \wedge \psi) \wedge\left(p \prec p^{\prime \prime \prime}\right) \wedge\left(q \prec q^{\prime \prime \prime}\right) \\
& \Leftrightarrow \quad \exists p^{\prime \prime \prime} q^{\prime \prime \prime} . A_{p^{\prime \prime \prime} \star q^{\prime \prime \prime}} \phi \wedge A_{p^{\prime \prime \prime} \star q^{\prime \prime \prime}} \psi \wedge\left(p \prec p^{\prime \prime \prime}\right) \wedge\left(q \prec q^{\prime \prime \prime}\right) \\
& \Rightarrow A_{p \times q} \phi \wedge A_{p \times q} \psi \Leftrightarrow \exists p^{\prime} q^{\prime} p^{\prime \prime} q^{\prime \prime} . A_{p^{\prime} \star q^{\prime}} \phi \wedge A_{p^{\prime \prime} \star q^{\prime \prime}} \psi \\
& \wedge\left(p \prec p^{\prime}\right) \wedge\left(p \prec p^{\prime \prime}\right) \wedge\left(q \prec q^{\prime}\right) \wedge\left(q \prec q^{\prime \prime}\right) .
\end{aligned}
$$

For the converse, we put $p^{\prime \prime \prime} \equiv p^{\prime} \star p^{\prime \prime}$ and $q^{\prime \prime \prime} \equiv q^{\prime} \star q^{\prime \prime}$ and need

$$
A_{p^{\prime} \star q^{\prime}} \phi \Rightarrow A_{p^{\prime} \star p^{\prime \prime} \star q^{\prime} \star q^{\prime \prime}} \phi \quad \text { and } \quad\left(p \preccurlyeq p^{\prime}\right) \wedge\left(p \preccurlyeq p^{\prime \prime}\right) \Rightarrow\left(p \preccurlyeq p^{\prime} \star p^{\prime \prime}\right) \text {, }
$$

which we have from contravariance of $A_{p}$ and the fact that we had an $\wedge$-basis. The new operation $\times$ can be defined for more factors in the same way, using recursion and equational induction.

General locally compact objects are a good deal more complicated than domains. The extension of the earlier results of this section rely heavily on the "lattice" structure $(0,1,+, \star)$, which we investigate next.

\section{The LATtice BASIS ON $\Sigma^{N}$}

In the next section we shall show that the properties of $\preccurlyeq$ listed in Section [1] are sufficient to reconstruct the object $X$ and its basis. To do this, however, we need some 
more technical information about the lattice basis on $\Sigma^{N}$, and about the free distributive lattice on $N$.

Remark 13.1. When an object $X$ has an $N$-indexed basis $\left(\beta^{n}, A_{n}\right)$ there is an embedding $X \longmapsto \Sigma^{N}$, given by Lemma 7.4 . This structure may be summed up by the diagram,

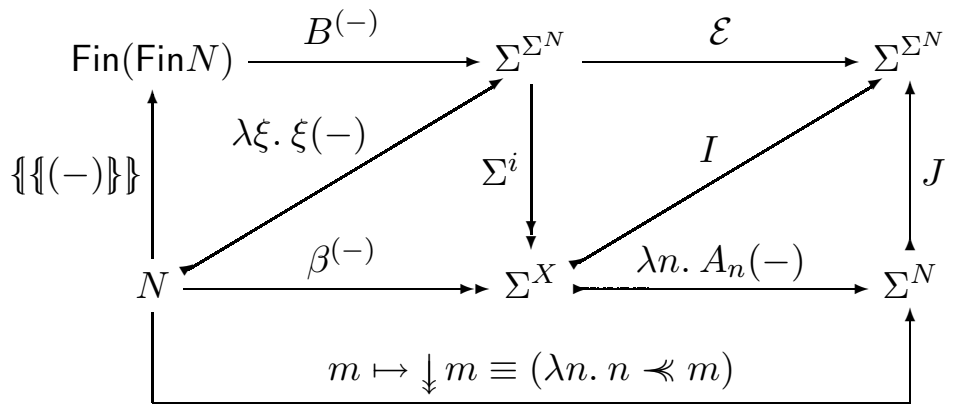

in which $\{-\}$ means the singleton list, the map $J$ on the right is defined by

$$
J \phi \equiv \lambda \xi . \exists n . \phi n \wedge \xi n,
$$

and $J \phi$ preserves $\vee, \perp$ and $\exists$. We define $\mathcal{E}$ from $\preccurlyeq$ using the lattice basis $\left(B^{L}, \mathcal{A}_{L}\right)$ on $\Sigma^{N}$.

Remark 13.2. These results may be seen as presentation of the algebra $\Sigma^{X}$. In this, $N$ is the set of generators and $\mathrm{DL}(N)$ is the free (imposed) distributive lattice on them, which we construct in Proposition 13.8 below. Then there are homomorphisms

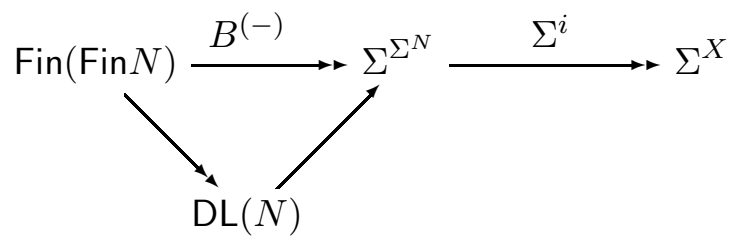

as $B^{(-)}$takes the imposed list operations $0,1,+$ and $\star$ to the intrinsic structure $\perp, \top, \vee$ and $\wedge$ in $\Sigma^{\Sigma^{N}}$, whilst $\Sigma^{i}$ preserves the latter in $\Sigma^{X}$. The relation $\prec$ encodes the system of "equations" that distinguishes the particular algebra $\Sigma^{X}$ from the generic one $\Sigma^{\Sigma^{N}}$ that is freely generated by $N$.

This explains why $n \preccurlyeq m$ does not imply $n \preccurlyeq m$ in our system, whereas $\alpha \ll \beta$ implies $\alpha \leq \beta$ in a continuous lattice. Topologically, we already saw the point in Remarks 1.8 and 6.15. many distinct codes may in principle represent the same open or compact subobject. To put this the other way round, since equality (or containment) of open subobjects is not computable, we cannot deduce equality (or comparison) of codes from semantic coincidence of subobjects.

Remark 13.3. The triangle

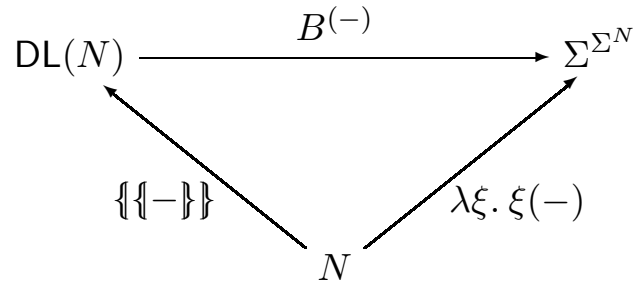

illustrates the comparison between the monad that captures the imposed $(0,1,+, \star)$ distributive lattice structure and the one in Axiom 3.3 based on $\Sigma^{(-)} \dashv \Sigma^{(-)}$. The upward 
maps are the units of these monads. We leave the interested student to construct the multiplication map of the DL-monad as a list program, $c f$. flatten in Lemma 8.6.

We are not quite justified in saying that the $\Sigma^{\Sigma^{(-)}}$monad defines the intrinsic $(\perp, \top, \vee, \wedge)$ distributive lattice structure. Corollary 10.3 said that the homomorphisms are the same, but I have not been able to show that every object whose intrinsic order is that of a distributive lattice is an algebra for the monad, i.e. of the form $\Sigma^{X}$ for some object $X$.

Notation 13.4. Recall from Proposition 8.8 that the basis on $\Sigma^{N}$ is

$$
B^{L} \xi \equiv \exists \ell \in L . \forall m \in \ell . \xi m \text { and } \mathcal{A}_{L} \Phi \equiv \forall \ell \in L . \Phi(\lambda m . m \in \ell),
$$

from which we obtain the way-below relation

$$
\mathcal{A}_{L} B^{R} \equiv\left(R \subset^{\sharp} L\right) \equiv \forall \ell \in L . \exists \ell^{\prime} \in R .\left(\ell^{\prime} \subset \ell\right) .
$$

The list of lists $R+S$ is given by concatenation, whilst $R \star S$ and $B^{R \star S}$ were defined in Lemma 8.6 .

Lemma 13.5. $\left(B^{L}, \mathcal{A}_{L}\right)$ is a lattice basis:

$$
\begin{gathered}
B^{1}=\top \quad B_{0}=\perp \quad B^{R \star S}=B^{R} \wedge B^{S} \quad B^{R+S}=B^{R} \vee B^{S} \\
\mathcal{A}_{0} \Phi \Leftrightarrow \top \quad \mathcal{A}_{L} \perp \Leftrightarrow(L=0) \quad \mathcal{A}_{R+S}=\mathcal{A}_{R} \wedge \mathcal{A}_{S} .
\end{gathered}
$$

Lemma 13.6. $\left(B^{L}, \mathcal{A}_{L}\right)$ is a filter basis: $A \top \Leftrightarrow \top$ and $\mathcal{A}_{L}(\Phi \wedge \Psi) \Leftrightarrow \mathcal{A}_{L} \Phi \wedge \mathcal{A}_{L} \Psi$.

Proof. $\quad \mathcal{A}_{L}(\Phi \wedge \Psi) \Leftrightarrow \forall \ell \in L .(\Phi \wedge \Psi)(\lambda m . m \in \ell)$

$$
\begin{aligned}
& \Leftrightarrow \quad \forall \ell \in L . \Phi(\lambda m . m \in \ell) \wedge \Psi(\lambda m . m \in \ell) \\
& \Leftrightarrow \quad(\forall \ell \in L . \Phi(\lambda m . m \in \ell)) \wedge(\forall \ell \in L . \Psi(\lambda m . m \in \ell)) \\
& \Leftrightarrow \mathcal{A}_{L} \Phi \wedge \mathcal{A}_{L} \Psi
\end{aligned}
$$

The Wilker condition says that we can split the list into the two parts that satisfy the respective disjuncts.

Lemma 13.7. $\quad \mathcal{A}_{L}(\Phi \vee \Psi) \Leftrightarrow \exists L_{1} L_{2} .\left(L=L_{1}+L_{2}\right) \wedge \mathcal{A}_{L_{1}} \Phi \wedge \mathcal{A}_{L_{2}} \Psi$.

Proposition 13.8. We write $L \cong R$ if both $R \subset^{\sharp} L$ and $L \subset^{\sharp} R$. This is an open congruence for the imposed structure on $\operatorname{Fin}(\operatorname{Fin} N)$, and the free imposed distributive lattice $\operatorname{DL}(N)$ is its quotient [C, Section 10].

Remark 13.9. So far we have not used any of the structure on $N$ itself. Since we have a lattice basis for $X$, by definition

$$
\beta^{(-)}: N \rightarrow \Sigma^{X}
$$

takes the imposed structure $(0,1,+, \star)$ on $N$ to the intrinsic structure $(\perp, \top, \vee, \wedge)$ on $\Sigma^{X}$. Associated with this imposed structure is an imposed order relation $\preccurlyeq$, which $\beta^{(-)}$takes to $\leq$, but with respect to which the dual basis $A_{(-)}$is contravariant.

Definition 13.10. We define $\preccurlyeq$ from + and $\star$ as the least relation such that

$$
\begin{array}{lll}
0 \preccurlyeq n \preccurlyeq n \preccurlyeq 1 & (k \star n)+(k \star m) \preccurlyeq k *(n+m) \\
\frac{n \preccurlyeq k \preccurlyeq m}{n \preccurlyeq m} \quad \frac{k \preccurlyeq m \quad k \preccurlyeq n}{k \preccurlyeq m \star n} \quad \frac{n \preccurlyeq k \quad m \preccurlyeq k}{n+m \preccurlyeq k}
\end{array}
$$

and again we write $n \cong m$ when both $n \preccurlyeq m$ and $m \preccurlyeq n$.

Proposition 13.11. The relation $\cong$ is an open congruence on $N$ whose quotient is an imposed distributive lattice. 
Notation 13.12. Returning to $\operatorname{Fin}(\operatorname{Fin} N), L$ is regarded as a formal sum of products of elements of $N$ (additive normal form). This may be "evaluated" by means of the operation

$$
\text { ev }: \operatorname{Fin}(\operatorname{Fin} N) \rightarrow N \text {. }
$$

This is defined for lists by a generalisation of Lemma 8.6. A similar construction works for Kuratowski-finite subsets instead, except that then $N$ has actually to satisfy the equations for a distributive lattice up to equality, and not just up to $\cong(c f$. Notation 4.9) . The map

$$
\operatorname{DL}(N) \equiv \operatorname{Fin}(\operatorname{Fin} N) /(\cong) \stackrel{\mathrm{ev} /(\cong)}{\longrightarrow} N /(\cong)
$$

is the structure map of the distributive lattice, regarded as an algebra for the DL-monad.

Proposition 13.13. The map ev : $\operatorname{Fin}(\operatorname{Fin} N) \rightarrow N$ is a homomorphism in the sense that $\mathrm{ev} 0=0$ and $\mathrm{ev} 1=1$ by construction, whilst

$$
\mathrm{ev}(R+S) \cong(\mathrm{ev} R)+(\mathrm{ev} S) \text { and } \mathrm{ev}(R \star S) \cong(\mathrm{ev} R) \star(\mathrm{ev} S) .
$$

Proof. This is a standard piece of universal algebra, which again we leave as a student exercise. The + equation is proved by list induction, using associativity and commutativity of + up to $\cong$. The equation for $\star$ is more difficult, as we have to take apart the inner lists, and use distributivity.

Lemma 13.14. $\mathcal{A}_{L} B^{L} \Leftrightarrow \top$ but

$$
\begin{aligned}
& \mathcal{A}_{L} B^{R} \Leftrightarrow\left(R \subset^{\sharp} L\right) \Leftrightarrow \forall \ell \in L . \exists \ell^{\prime} \in R .\left(\ell^{\prime} \subset \ell\right) \quad \Rightarrow \quad(\mathrm{ev} L \preccurlyeq \mathrm{ev} R) \\
& \mathcal{A}_{L}(\lambda \xi . \xi n) \Leftrightarrow \forall \ell \in L . n \in \ell \quad \Rightarrow \quad(\mathrm{ev} L \preccurlyeq n) \\
& \mathcal{A}_{L}(\lambda \xi . \xi n \wedge \xi m) \Leftrightarrow \forall \ell \in L . n \in \ell \wedge m \in \ell \quad \Rightarrow \quad(\mathrm{ev} L \preccurlyeq n \star m) \\
& \mathcal{A}_{L}(\lambda \xi . \xi n \vee \xi m) \Leftrightarrow \forall \ell \in L . n \in \ell \vee m \in \ell \quad \Rightarrow \quad(\mathrm{ev} L \preccurlyeq n+m)
\end{aligned}
$$

with equality in the cases $L \equiv\{\{k\}\}$ and $L \equiv R$.

Proof. In the expansion of $\mathcal{A}_{L} B^{R}$, the products in $L$ are of longer strings than those in $R$. The other three results follow by putting $R \equiv\{\{n\}\},\{\{n, m\}\}$ and $\{\{n\},\{m\}\}$,

Remark 13.15. The foregoing discussion of $\cong$ is the price that we pay for not requiring $(N, 0,1,+, \star)$ to satisfy the equations for a distributive lattice in Remark 1.8. If, like [JS96, we had done so, we would have instead paid the same price to construct the basis for $\Sigma^{X}$. This is indexed by the free distributive lattice on $N^{\mathrm{op}} q u \hat{a} \star$-semilattice, i.e. with new joins but using the old ones as meets. What we have bought for this price is the flexibility of switching amongst various kinds of basis (Definition [6.6), when we needed or didn't need them, throughout the paper.

The reason why it is unnecessary to form the quotient of $N$ or $\operatorname{Fin}(\operatorname{Fin} N)$ by the congruence $\cong$ is that we never deal with their elements up to equality. The things that matter are the rules

$$
\frac{n \cong n^{\prime} \quad n \preccurlyeq k}{n^{\prime} \preccurlyeq k} \quad \frac{k \preccurlyeq n \quad n \cong n^{\prime}}{k \nVdash n^{\prime}}
$$

which are examples of Corollary 11.5. Indeed the relation $\preccurlyeq$ itself is only needed to avoid the extra rules that relate + and $\star$ to $\preccurlyeq$, which appear in [JS96, Lemma 7]. 


\section{From the basis to the SPACE}

We are now able to show that any "abstract" basis satisfying the conditions of Section 11 actually arises from some definable object. As in Section [12 we have to define a nucleus $\mathcal{E}: \Sigma^{\Sigma^{N}} \rightarrow \Sigma^{\Sigma^{N}}$ and (in the next section) characterise its admissible terms in the sense of Definition 12.8.

Definition 14.1. An abstract basis is an overt discrete object $N$ with elements $0,1 \in N$, binary operations $+, \star: N \times N \rightarrow N$ and an open binary relation $\preccurlyeq: N \times N \rightarrow \Sigma$ such that

$$
\begin{aligned}
& 0 \preccurlyeq 0 \quad \frac{n \preccurlyeq p \quad m \preccurlyeq p}{n+m \preccurlyeq p} \quad \frac{m^{\prime} \preccurlyeq m \quad m \preccurlyeq n \quad n \preccurlyeq n^{\prime}}{m^{\prime} \preccurlyeq n^{\prime}} \quad \frac{n \preccurlyeq m}{\overline{n \preccurlyeq k \preccurlyeq m}} \\
& \frac{n \preccurlyeq m \quad m \preccurlyeq p \quad m \preccurlyeq q}{n \preccurlyeq p \star q} \quad \frac{n \preccurlyeq p+q}{\overline{n \preccurlyeq p^{\prime}+q^{\prime} \quad p^{\prime} \preccurlyeq p \quad q^{\prime} \preccurlyeq q}}
\end{aligned}
$$

where $\preccurlyeq$ is defined from + and $\star$ by Definition 13.10

Notation 14.2.

$$
\begin{aligned}
\mathcal{E} & \equiv \lambda \Phi \cdot \lambda \xi \cdot J\left(\lambda n . \exists L \cdot(n \preccurlyeq \mathrm{ev} L) \wedge \mathcal{A}_{L} \Phi\right) \xi \\
& \equiv \lambda \Phi \cdot \lambda \xi \cdot \exists n \cdot \exists L \cdot \xi n \wedge(n \preccurlyeq \mathrm{ev} L) \wedge \mathcal{A}_{L} \Phi .
\end{aligned}
$$

We have to show that this satisfies the equations in Theorem 10.10

$$
\Phi, \Psi: \Sigma^{\Sigma^{N}} \vdash \mathcal{E}(\Phi \wedge \Psi)=\mathcal{E}(\mathcal{E} \Phi \wedge \mathcal{E} \Psi) \text { and } \mathcal{E}(\Phi \vee \Psi)=\mathcal{E}(\mathcal{E} \Phi \vee \mathcal{E} \Psi)
$$

This is made a little easier by the fact that we need only test these equations for basic $\Phi \equiv B^{R}$ and $\Psi \equiv B^{S}$ :

Lemma 14.3. $\frac{R, S: \operatorname{Fin}\left(\operatorname{Fin}(N) \vdash \mathcal{E}\left(\mathcal{E} B^{R} \odot \mathcal{E} B^{S}\right)=\mathcal{E}\left(B^{R} \odot B^{S}\right)\right.}{\Phi, \Psi: \Sigma^{\Sigma^{N}} \vdash \mathcal{E}(\mathcal{E} \Phi \odot \mathcal{E} \Psi)=\mathcal{E}(\Phi \odot \Psi) .}$

Proof. We use the lattice basis expansion $\Phi=\exists R . \mathcal{A}_{R} \Phi \wedge B^{R}$.

Note first that the combined expansion using distributivity (Lemma 11.8),

$$
\Phi \odot \Psi=\exists R S . \mathcal{A}_{R} \Phi \wedge \mathcal{A}_{S} \Psi \wedge\left(B^{R} \odot B^{S}\right),
$$

is directed in $\langle R, S\rangle$, so $\mathcal{E}$ preserves the join by Theorem 9.6] and $\mathcal{E} \Phi=\exists R . \mathcal{A}_{R} \Phi \wedge \mathcal{E} B^{R}$. Using distributivity, directedness and Scott continuity again, we have

$$
\begin{array}{rlr}
\mathcal{E} \Phi \odot \mathcal{E} \Psi & =\exists R S . \mathcal{A}_{R} \Phi \wedge \mathcal{A}_{S} \Psi \wedge\left(\mathcal{E} B^{R} \odot \mathcal{E} B^{S}\right) & \text { distributivity } \\
\mathcal{E}(\mathcal{E} \Phi \odot \mathcal{E} \Psi) & =\exists R S . \mathcal{A}_{R} \Phi \wedge \mathcal{A}_{S} \Psi \wedge \mathcal{E}\left(\mathcal{E} B^{R} \odot \mathcal{E} B^{S}\right) & \text { directedness } \\
& =\exists R S . \mathcal{A}_{R} \Phi \wedge \mathcal{A}_{S} \Psi \wedge \mathcal{E}\left(B^{R} \odot B^{S}\right) & \text { hypothesis } \\
& =\mathcal{E}(\Phi \odot \Psi)
\end{array}
$$

Next we need to evaluate the expression $\mathcal{A}_{L}\left(\mathcal{E} B^{R}\right)$.

Lemma 14.4. $\quad \mathcal{E} B^{R}=J(\lambda n . n \preccurlyeq \mathrm{ev} R)$.

Proof.

$$
\begin{array}{rlr}
\mathcal{E} B^{R} & =J\left(\lambda n . \exists L . n \preccurlyeq \mathrm{ev} L \wedge \mathcal{A}_{L} B^{R}\right) & \text { Definition 14.2 } \\
& \leq J(\lambda n . \exists L . n \preccurlyeq \mathrm{ev} L \preccurlyeq \mathrm{ev} R) & \text { Lemma 13.14 } \\
& =J(\lambda n . n \preccurlyeq \mathrm{ev} R) & \text { Definition 14.1 }
\end{array}
$$

but the $\leq$ is an equality, as we may put $L \equiv R$ in the other direction. 
Lemma 14.5. Since, by hypothesis, « satisfies $0 \prec k$ and (cf. Lemma [11.3)

$$
\frac{n \preccurlyeq k \quad m \preccurlyeq k}{n+m \preccurlyeq k} \quad \frac{m \star n \preccurlyeq n \quad n \preccurlyeq r}{m \star n \preccurlyeq r}
$$

we have $\mathcal{A}_{L}\left(\mathcal{E} B^{R}\right) \Rightarrow(\mathrm{ev} L \preccurlyeq \mathrm{ev} R)$, with equality in the case $L \equiv\{\{k\}\}$.

Proof. The reason for the inequality is that, whereas $B^{(-)}: \operatorname{Fin}(\operatorname{Fin} N) \rightarrow \operatorname{DL}(N) \rightarrow \Sigma^{\Sigma^{N}}$ sends + to $\vee$ and $\star$ to $\wedge, \mathcal{A}_{(-)}$only takes + to $\wedge$, not necessarily $\star$ to $\vee$.

$$
\begin{aligned}
& \mathcal{A}_{L}\left(\mathcal{E} B^{R}\right) \Leftrightarrow \forall \ell \in L . \mathcal{E} B^{R}(\lambda m . m \in \ell) \\
& \operatorname{def} \mathcal{A}_{L} \\
& \Leftrightarrow \quad \forall \ell \in L . J(\lambda n . n \preccurlyeq \operatorname{ev} R)(\lambda m . m \in \ell) \\
& \Leftrightarrow \quad \forall \ell \in L . \exists n \in \ell . n \nVdash \mathrm{ev} R \\
& \text { Lemma } 14.4 \\
& \Rightarrow \quad \forall \ell \in L . \mu \ell \preccurlyeq \mathrm{ev} R \\
& \text { def. } J \\
& \Leftrightarrow \mathrm{ev} L \preccurlyeq \mathrm{ev} R \\
& \star \text { rule } \\
& 0,+ \text { rules }
\end{aligned}
$$

Here $\mu \ell$ is the "product" of $\ell$, in the sense of 1 and $\star$ (written fold $\star 1 \ell$ in functional programming notation), so $n \in \ell \Rightarrow \mu \ell \preccurlyeq n$. Then $\operatorname{ev} L$ is the sum of these products (Definition 13.10). Equality holds when $L \equiv\{\{k\}\}$ since $\ell=\{k\}$ and $\operatorname{ev} L=\mu \ell=k$.

Equipped with formulae for $\mathcal{E} B^{R}$ and $\mathcal{A}_{L}\left(\mathcal{E} B^{R}\right)$, we can now verify the two equations. Their proofs are almost the same, illustrating once again the lattice duality that we get by putting directed joins into the background. Unfortunately, they're not quite close enough for us to use $\odot$ and give just one proof. First, however, we give the similar but slightly simpler argument for idempotence, although it is easily seen to be implied by either of the other results.

Proposition 14.6. If $\nleftarrow$ satisfies the transitive and interpolation rules,

$$
\frac{n \nVdash r}{\overline{n \nVdash m \preccurlyeq r}}
$$

cf. Corollary 11.7, then $\mathcal{E}$ is idempotent: $\mathcal{E}(\mathcal{E} \Phi)=\mathcal{E} \Phi$.

Proof. By (a simpler version of) Lemma 14.3 it's enough to consider $\Phi \equiv B^{R}$,

$$
\begin{aligned}
& \mathcal{E}\left(\mathcal{E} B^{R}\right)=J\left(\lambda n . \exists L .(n \preccurlyeq \operatorname{ev} L) \wedge \mathcal{A}_{L}\left(\mathcal{E} B^{R}\right)\right) \quad \text { Notation } 14.2 \\
& \leq J(\lambda n . \exists m .(n \ll m) \wedge(m \nVdash \mathrm{ev} R)) \quad \text { Lemma 14.5 } \\
& =J(\lambda n . n \gtrless \mathrm{ev} R) \quad \text { hypothesis } \\
& =\mathcal{E} B^{R} \quad \text { Lemma 14.4 }
\end{aligned}
$$

where $m \equiv \mathrm{ev} L$. However, the $\leq$ is an equality as we may use $L \equiv\{\{m\}\}$ in Lemma 14.4 to prove $\geq$.

Proposition 14.7. Since $\preccurlyeq$ obeys the rule linking it with $\star$,

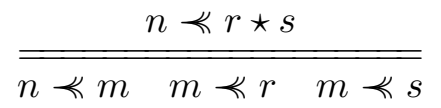

(cf. Lemma 11.11) $\mathcal{E}$ satisfies the $\wedge$-equation, $\mathcal{E}(\Phi \wedge \Psi)=\mathcal{E}(\mathcal{E} \Phi \wedge \mathcal{E} \Psi)$. 
Proof. By Lemma 14.3, it's enough to consider $\Phi \equiv B^{R}$ and $\Psi \equiv B^{S}$. With $m \equiv \operatorname{ev} L$, $r \equiv \mathrm{ev} R$ and $s \equiv \mathrm{ev} S$,

$$
\begin{aligned}
& \mathcal{E}\left(\mathcal{E} B^{R} \wedge \mathcal{E} B^{S}\right)=J\left(\lambda n . \exists L .(n \nVdash \mathrm{ev} L) \wedge \mathcal{A}_{L}\left(\mathcal{E} B^{R} \wedge \mathcal{E} B^{S}\right)\right) \quad \text { Notation } 14.2 \\
& =J\left(\lambda n . \exists L .(n \nVdash \mathrm{ev} L) \wedge \mathcal{A}_{L}\left(\mathcal{E} B^{R}\right) \wedge \mathcal{A}_{L}\left(\mathcal{E} B^{S}\right)\right) \quad \text { Lemma 13.6 } \\
& \leq J(\lambda n . \exists m .(n \nVdash m) \wedge(m \nVdash r) \wedge(m \nVdash s)) \quad \text { Lemma 14.5 } \\
& =J(\lambda n \cdot n \preccurlyeq r \star s) \\
& =J(\lambda n \cdot n \nVdash \mathrm{ev}(R \star S)) \\
& \text { hypothesis } \\
& =\mathcal{E} B^{R \star S}=\mathcal{E}\left(B^{R} \wedge B^{S}\right)
\end{aligned}
$$

To make the $\leq$ an equality, we use $L \equiv\{\{m\}\}$ in Lemma 13.14,

Proposition 14.8. If $\ll$ satisfies the Wilker rule linking it with + ,

$$
\frac{n \preccurlyeq r+s}{\overline{n \ll p+q \quad p \ll r \quad q \preccurlyeq s}}
$$

(cf. Lemma 11.12) then $\mathcal{E}$ satisfies the $\vee$-equation, $\mathcal{E}(\Phi \vee \Psi)=\mathcal{E}(\mathcal{E} \Phi \vee \mathcal{E} \Psi)$.

Proof. With $r \equiv \mathrm{ev} R, s \equiv \mathrm{ev} S, p \equiv \operatorname{ev} L_{1}, q \equiv \operatorname{ev} L_{2}$ and $L=L_{1}+L_{2}$,

$$
\begin{aligned}
& \mathcal{E}\left(\mathcal{E} B^{R} \vee \mathcal{E} B^{S}\right)=J\left(\lambda n . \exists L .(n \preccurlyeq \operatorname{ev} L) \wedge \mathcal{A}_{L}\left(\mathcal{E} B^{R} \vee \mathcal{E} B^{S}\right)\right) \quad \text { Notation } 14.2 \\
& =J\left(\lambda n . \exists L_{1} L_{2} \cdot\left(n \ll \operatorname{ev} L_{1}+\operatorname{ev} L_{2}\right) \wedge \mathcal{A}_{L_{1}}\left(\mathcal{E} B^{R}\right) \wedge \mathcal{A}_{L_{2}}\left(\mathcal{E} B^{S}\right)\right) 13.7 \\
& \leq J(\lambda n . \exists p q .(n \preccurlyeq p+q) \wedge(p \preccurlyeq r) \wedge(q \prec s)) \quad \text { Lemma 14.5 } \\
& =J(\lambda n . n \nvdash r+s) \quad \text { hypothesis } \\
& =J(\lambda n \cdot n \preccurlyeq \operatorname{ev}(R+S)) \quad \text { Lemma } 13.13 \\
& =\mathcal{E} B^{R+S}=\mathcal{E}\left(B^{R} \vee B^{S}\right) \quad \text { Lemmas 14.4 \& 13.5 }
\end{aligned}
$$

Again the $\leq$ becomes an equality, using $L_{1} \equiv\{\{p\}\}, L_{2} \equiv\{\{q\}\}$ and $L \equiv L_{1}+L_{2}$ in Lemma 13.14.

Theorem 14.9. $\mathcal{E}$ is a nucleus on $\Sigma^{N}$ in the sense of $[\mathrm{B}$, Section 8].

\section{The POINTS OF THE NEW SPACE}

Now we can characterise the (parametric) points of the object that we constructed from an abstract basis in the previous section. Then we define a lattice basis on it, just as we did for continuous dcpos in Theorem 12.11.

Notation 15.1. We are again in the situation of Definition 12.8 that we have defined a nucleus $\mathcal{E}$ from an abstract basis, so the monadic calculus [B, $\S 8]$ provides a subobject $i:\left\{\Sigma^{N} \mid \mathcal{E}\right\} \longmapsto \Sigma^{N}$ with $\Sigma$-splitting $I$. As in Lemma 12.9 the next task is to characterise its elements, i.e. those $\Gamma \vdash \xi: \Sigma^{N}$ that are admissible:

$$
\Gamma, \Phi: \Sigma^{\Sigma^{N}} \vdash \Phi \xi \Leftrightarrow \mathcal{E} \Phi \xi .
$$

Lemma 15.2. If $\Gamma \vdash \xi: \Sigma^{N}$ is admissible then it is rounded in the sense that

$$
\Gamma, n: N \vdash \xi n \Leftrightarrow \exists m . \xi m \wedge m \prec n .
$$


Proof. Consider $\Phi \equiv \lambda \xi$. $\xi n$, so $\mathcal{A}_{L} \Phi \Rightarrow(\mathrm{ev} L \preccurlyeq n)$ by Lemma 13.14. Then

$$
\begin{array}{rlr}
\xi n \Leftrightarrow \Phi \xi \Leftrightarrow \mathcal{E} \Phi \xi & \Leftrightarrow \exists m . \exists L . \xi m \wedge(m \preccurlyeq \mathrm{ev} L) \wedge \mathcal{A}_{L} \Phi & \text { Notation 14.2 } \\
& \Rightarrow \exists m . \exists L . \xi m \wedge(m \prec \mathrm{ev} L \preccurlyeq n) & \text { above } \\
& \Rightarrow \exists m . \xi m \wedge(m \prec n) & \text { monotonicity }
\end{array}
$$

where $\Rightarrow$ is actually equality, as we may put $L \equiv\{\{n\}\}$ in Lemma 13.14 to obtain $\Leftarrow$.

Lemma 15.3. If $\Gamma \vdash \xi: \Sigma^{N}$ is admissible then it is a lattice homomorphism in the sense that

$$
\xi 0 \Leftrightarrow \perp \quad \xi 1 \Leftrightarrow \top \quad \xi(n+m) \Leftrightarrow \xi n \vee \xi m \quad \xi(n \star m) \Leftrightarrow \xi n \wedge \xi m .
$$

Proof. Consider $\Phi \equiv \lambda \xi$. $\xi n \odot \xi m$, so $\mathcal{A}_{L} \Phi \Rightarrow(\mathrm{ev} L \preccurlyeq n \odot m)$ by Lemma 13.14. Then

$$
\begin{array}{rlr}
\xi n \odot \xi m & \Leftrightarrow \Phi \xi \Leftrightarrow \mathcal{E} \Phi \xi & \\
& \Leftrightarrow \exists k . \exists L \cdot \xi k \wedge(k \prec \mathrm{ev} L) \wedge \mathcal{A}_{L} \Phi & \text { Notation 14.2 } \\
& \Rightarrow \exists k \cdot \exists L . \xi k \wedge(k \preccurlyeq \mathrm{ev} L \preccurlyeq n \odot m) & \text { above } \\
& \Rightarrow \exists k . \xi k \wedge(k \prec n \odot m) \Leftrightarrow \xi(n \odot m) & \text { roundedness }
\end{array}
$$

with equality by $L \equiv\{\{n \odot m\}\}$ in Lemma 13.14 Similarly, for the constants, consider $\Phi \equiv \lambda \xi$. T, so $\mathcal{A}_{L} \Phi \Leftrightarrow \top \Leftrightarrow(\mathrm{ev} L \preccurlyeq 1)$, and $\Phi \equiv \lambda \xi$. $\perp$, so $\mathcal{A}_{L} \Phi \Leftrightarrow \forall \ell \in L . \perp \Leftrightarrow(L=0)$.

Notice that it is the fact that $\xi$ is rounded (for $\preccurlyeq$ ), rather than a homomorphism (for $0,1,+, \star)$, that distinguishes the particular object $X$ from the ambient $\Sigma^{N}$ into which it is embedded, $c f$. Remark 13.2

Lemma 15.4. If $\Gamma \vdash \xi: \Sigma^{N}$ is a rounded lattice homomorphism then it is admissible.

Proof.

$$
\begin{array}{rlr}
\Phi \xi & \Leftrightarrow \exists L . \mathcal{A}_{L} \Phi \wedge B^{L} \xi & \text { lattice basis on } \Sigma^{N} \\
& \Leftrightarrow \exists L . \mathcal{A}_{L} \Phi \wedge \xi(\mathrm{ev} L) & \\
& \Leftrightarrow \exists L . \exists n . \mathcal{A}_{L} \Phi \wedge \xi n \wedge(n \prec \mathrm{ev} L) & \text { rounded } \\
& \Leftrightarrow \mathcal{E} \Phi \xi & \text { Definition 14.2 }
\end{array}
$$

where the second step exploits the definition of $B^{L}$ (Lemma 13.5) and ev $L$ when $\xi$ is a homomorphism.

Remark 15.5. Hence the rounded lattice homomorphisms $\xi: \Sigma^{N}$ are the points of $X$, whilst the rounded filters ( $\wedge$-homomorphisms) correspond to its compact saturated subspaces, at least when $X$ is a classical stably locally compact space (Example 12.13).

Lemma 15.6. $\beta^{n} \equiv \lambda x$. ixn and $A_{n} \equiv \lambda \phi . I \phi\{n\}$ provide an effective basis for $X$.

Proof. First note that $\xi \mapsto J \phi \xi$ preserves joins in $\xi$, and therefore so do $\xi \mapsto \mathcal{E} \Phi \xi$ and $\xi \mapsto I \phi \xi$, as required by Lemma [7.7] so we recover

$$
i x=\lambda n \cdot \beta^{n} x \text { and } I \phi=\lambda \xi \cdot \exists n . A_{n} \phi \wedge \xi n .
$$


For the basis expansion, let $\phi: \Sigma^{X}$ and $x: X$. Then $\xi \equiv i x: \Sigma^{N}$ is admissible, and $\phi=\Sigma^{i} \Phi$, where $\Phi \equiv I \phi: \Sigma^{\Sigma^{N}}$.

$$
\begin{array}{rlr}
\exists n . A_{n} \phi \wedge \beta^{n} x & \Leftrightarrow \exists n . I \phi\{n\} \wedge i x n & \operatorname{defs} \beta^{n}, A_{n} \\
& \Leftrightarrow \exists n .\left(I \cdot \Sigma^{i}\right) \Phi\{n\} \wedge \xi n & \operatorname{defs} \Phi, \xi \\
& \Leftrightarrow \exists n . \mathcal{E} \Phi\{n\} \wedge \xi n & \operatorname{defs} i, I \\
& \Leftrightarrow \mathcal{E} \Phi \xi & \mathcal{E} \Phi \text { preserves } \xi \Leftrightarrow \exists n .\{n\} \wedge \xi n \\
& \Leftrightarrow \Phi \xi & \xi \text { admissible } \\
& \Leftrightarrow(I \phi)(i x) & \text { defs } \Phi, \xi \\
& \Leftrightarrow \phi x
\end{array}
$$

Corollary 15.7. $x=$ focus $\left(\exists n . A_{n} \wedge \xi n\right)$.

This is the generalisation of $x=\bigvee\left\{p_{n} \mid \xi n\right\}$ in domain theory (Proposition 12.3), and the version in ASD of $\{x\}=\bigcap K\{x\} \preccurlyeq K$ in Theorem [5.18. The predicate $\xi n$ means $x \in U^{n} \subset K^{n}$ in the spatial setting, $c f$. Definition 1.1.

Theorem 15.8. $\left(\beta^{n}, A_{n}\right)$ is a lattice basis, and its way-below relation is $\preccurlyeq$.

Proof. Since $x: X \vdash \xi \equiv i x \equiv \lambda n . \beta^{n} x: \Sigma^{N}$ is admissible, and therefore a homomorphism by Lemma 15.3, we have

$$
\beta^{0}=\lambda x . \perp, \quad \beta^{1}=\lambda x . \top \quad \text { and } \quad \beta^{n \odot m}=\beta^{n} \odot \beta^{m} .
$$

Next we check the equations on $A_{n}$ for an $\vee$-basis. Let $\phi: \Sigma^{X}$ and $\Phi \equiv I \phi: \Sigma^{\Sigma^{N}}$. Then $\mathcal{E} \Phi=I \cdot \Sigma^{i} \cdot I \phi=I \phi=\Phi$, so

$$
A_{n} \phi \Leftrightarrow I \phi\{n\} \Leftrightarrow \Phi\{n\} \Leftrightarrow \mathcal{E} \Phi\{n\} .
$$

Hence

$$
\begin{aligned}
& A_{0} \phi \quad \Leftrightarrow \mathcal{E} \Phi\{0\} \\
& \Leftrightarrow \quad \exists L .(0 \prec \operatorname{ev} L) \wedge \mathcal{A}_{L} \Phi \\
& \Leftarrow(0 \prec 0) \wedge \mathcal{A}_{0} \Phi \Leftrightarrow \top \\
& A_{n} \phi \wedge A_{m} \phi \Leftrightarrow \mathcal{E} \Phi\{n\} \wedge \mathcal{E} \Phi\{m\} \\
& \Leftrightarrow \quad \exists L_{1} L_{2} .\left(n \preccurlyeq \mathrm{ev} L_{1}\right) \wedge\left(m \prec \mathrm{ev} L_{2}\right) \wedge \mathcal{A}_{L_{1}} \Phi \wedge \mathcal{A}_{L_{2}} \Phi \quad \text { Def. } 14.2 \\
& \Rightarrow \quad \exists L_{1} L_{2} .\left(n+m \preccurlyeq \operatorname{ev} L_{1}+\mathrm{ev} L_{2}\right) \wedge \mathcal{A}_{L_{1}+L_{2}} \Phi \quad \text { Lemma 13.5] } \\
& \Rightarrow \quad \exists L .(n+m \prec \operatorname{ev} L) \wedge \mathcal{A}_{L} \Phi \quad \text { Lemma 13.13 } \\
& \Leftrightarrow \mathcal{E} \Phi\{n+m\} \Leftrightarrow A_{n+m} \phi \quad \text { Notation 14.2 }
\end{aligned}
$$

using distributivity, $L=L_{1}+L_{2}$ and Lemma 13.6. But $A_{n+m} \phi \Rightarrow A_{n} \phi$, so we have equality. Finally,

$$
\begin{aligned}
A_{n} \beta^{m} & \Leftrightarrow I(\lambda x . i x m)\{n\} \Leftrightarrow\left(I \cdot \Sigma^{i}\right)(\lambda \xi . \xi m)\{n\} & & \text { Lemma 15.6 } \\
& \Leftrightarrow \mathcal{E}(\lambda \xi . \xi m)\{n\} & & \\
& \Leftrightarrow \exists L .(n \preccurlyeq \mathrm{ev} L) \wedge \mathcal{A}_{L}(\lambda \xi . \xi m) & & \text { Notation 14.2 } \\
& \Rightarrow \exists L .(n \preccurlyeq \mathrm{ev} L \preccurlyeq m) & & \text { Lemma 13.14 } \\
& \Rightarrow(n \preccurlyeq m) & & \text { monotonicity }
\end{aligned}
$$

where we also obtain $\Leftarrow$ by putting $L \equiv\{\{m\}\}$ in Lemma 13.14, 
Corollary 15.9. If the object $X$ and its lattice basis $\left(\beta^{n}, A_{n}\right)$ had been given, and and $\mathcal{E}$ derived from them, this construction would recover $X$ and $\left(\beta^{n}, A_{n}\right)$ up to unique isomorphism. In particular, if we had started with a filter lattice basis, we would get it back.

We have shown that the notion of "abstract basis" is a complete axiomatisation of the way-below relation, and is therefore the formulation of the consistency requirements in Definitions [1.5 and 2.3. without using a classically defined topological space or locale as a reference.

This completes the proof of the equivalence amongst the characterisations of local compactness that we listed in the Introduction

In Lemmas 15.2 ff, we have also characterised points $x: X$ as rounded lattice homomorphisms $\xi: \Sigma^{N}$. We shall replace the predicate $\xi m$ by a binary relation $\widehat{H}_{n}^{m}$ in the next section, in order to generalise from points of $X$ to continuous functions $Y \rightarrow X$.

\section{Morphisms AS MATrices}

The analogy between bases for topology and bases for linear algebra in Section [6 can also be applied to morphisms. In this section we identify the abstract conditions satisfied by the relation

$$
f K^{n} \subset U^{m} \text { or } K^{n} \subset f^{*} U^{m}
$$

that was used in Definitions 1.6 and 2.3. Like , this is a binary relation between the two overt discrete objects of codes.

As this condition is an observable property of the function, it determines an open subobject of the set of functions $X \rightarrow Y$, and such properties form a sub-basis of the compact-open topology. If $X$ is locally compact then this object is the exponential $Y^{X}$ in both traditional topology and locale theory, and the conditions below are those listed in [Joh82, Lemma VII 4.11]. Unfortunately, there need not be a corresponding dual basis of compact subobjects to make $Y^{X}$ locally compact.

In our notation, the relation $f K^{n} \subset U^{m}$ is $A_{n}\left(\Sigma^{f} \beta^{m}\right)$. Clearly this question can easily be generalised to $A_{n}\left(H \beta^{m}\right)$, in which we replace $f$ by the "second class" map $\widehat{H}$ (Notation [5.13).

Notation 16.1. Let $\left(\beta^{n}, A_{n}\right)$ and $\left(\gamma^{m}, D_{m}\right)$ be $\vee$-bases for objects $X$ and $Y$ respectively. Then any first or second class morphism $\widehat{H}: X \rightarrow Y$ in $\mathrm{HS}$ (that is, $H: \Sigma^{Y} \rightarrow \Sigma^{X}$ in $\mathcal{S}$ ) has a matrix,

$$
\widehat{H}_{n}^{m} \equiv A_{n}\left(H \gamma^{m}\right): \Sigma
$$

which generalises the representation of $y: Y$ via $H \psi \equiv \psi x$ as $\xi \equiv i y \equiv \lambda y \cdot \gamma^{m} y$ in Lemmas 15.2fr.

Lemma 16.2. $H$ is recovered from $\widehat{H}_{n}^{m}$ as $H \psi=\exists m n . D_{m} \psi \wedge \widehat{H}_{n}^{m} \wedge \beta^{n}$.

Proof.

$$
\begin{aligned}
H \psi & =H\left(\exists m . D_{m} \psi \wedge \gamma^{m}\right) & \left(\gamma^{m}, D_{m}\right) \text { basis for } Y \\
& =\exists m . D_{m} \psi \wedge H \gamma^{m} & \text { indeed } \vee \text {-basis } \\
& =\exists m . D_{m} \psi \wedge \exists n . A_{n}\left(H \gamma^{m}\right) \wedge \beta^{n} & \left(\beta^{n}, A_{n}\right) \text { basis for } X \\
& =\exists m n . D_{m} \psi \wedge \widehat{H}_{n}^{m} \wedge \beta^{n} & \text { definition } \square
\end{aligned}
$$


Lemma 16.3. The matrix is directed in n, cf. Lemma 11.3:

$$
\widehat{H}_{0}^{m} \Leftrightarrow \top \quad \text { and } \quad \widehat{H}_{n+p}^{m} \Leftrightarrow \widehat{H}_{n}^{m} \wedge \widehat{H}_{p}^{m} .
$$

Proof. These are $A_{0} \phi \Leftrightarrow \top$ and $A_{n+p} \phi \Leftrightarrow A_{n} \phi \wedge A_{p} \phi$ with $\phi=H \gamma^{m}$. They hold because $\left(\beta^{n}, A_{n}\right)$ is an $\vee$-basis (Definition 6.6(a)).

Lemma 16.4. The matrix is monotone in $m$, cf. Lemma 11.6;

$$
\left(n^{\prime} \preccurlyeq X n\right) \wedge \widehat{H}_{n}^{m} \wedge\left(m \preccurlyeq{ }_{Y} m^{\prime}\right) \Rightarrow \widehat{H}_{n^{\prime}}^{m^{\prime}} .
$$

Proof. $\quad A_{n} \leq A_{n^{\prime}}$ (though we already had this from directedness) and $\gamma^{m} \leq \gamma^{m^{\prime}}$.

Lemma 16.5. The matrix is rounded, respecting on both sides, cf. Lemmas 11.7 8 [15.2]:

$$
\exists m^{\prime} .\left(m^{\prime} \preccurlyeq Y m\right) \wedge \widehat{H}_{n}^{m^{\prime}} \Leftrightarrow \widehat{H}_{n}^{m} \Leftrightarrow \exists n^{\prime} . \widehat{H}_{n^{\prime}}^{m} \wedge\left(n \preccurlyeq X n^{\prime}\right) .
$$

Proof.

$$
\begin{array}{rlr}
\widehat{H}_{n}^{m^{\prime}} & \Leftrightarrow A_{n}\left(H \gamma^{m^{\prime}}\right) & \\
& \Leftrightarrow A_{n}\left(H\left(\exists m \cdot D_{m} \gamma^{m^{\prime}} \wedge \gamma^{m}\right)\right) & \left(\gamma^{m}, D_{m}\right) \text { basis for } Y \\
& \Leftrightarrow \exists m \cdot D_{m} \gamma^{m^{\prime}} \wedge A_{n}\left(H \gamma^{m}\right) & \text { indeed, } \vee \text {-basis } \\
& \Leftrightarrow \exists m \cdot\left(m \preccurlyeq m^{\prime}\right) \wedge \widehat{H}_{n}^{m} & \\
\widehat{H}_{n^{\prime}}^{m} & \Leftrightarrow A_{n^{\prime}}\left(H \gamma^{m}\right) & \\
& \Leftrightarrow A_{n^{\prime}}\left(\exists n \cdot A_{n}\left(H \gamma^{m}\right) \wedge \beta^{n}\right) & \left(\beta^{n}, A_{n}\right) \text { basis for } X \\
& \Leftrightarrow \exists n \cdot A_{n}\left(H \gamma^{m}\right) \wedge A_{n^{\prime}} \beta^{n} & \text { indeed, } \vee \text {-basis } \\
& \Leftrightarrow \exists n . \widehat{H}_{n}^{m} \wedge\left(n^{\prime} \preccurlyeq n\right)
\end{array}
$$

Lemma 16.6. Suppose $n, m: N \vdash \rho(n, m): \Sigma$ satisfies the foregoing properties, i.e.

$$
\rho(0, k) \quad \frac{\rho(n, k) \quad \rho(m, k)}{\rho(n+m, k)} \quad \frac{n \preccurlyeq n^{\prime} \quad \rho\left(n^{\prime}, m\right)}{\rho(n, m)} \quad \frac{\rho\left(n, m^{\prime}\right) \quad m^{\prime} \ll m}{\rho(n, m)}
$$

and define $H: X \rightarrow Y$ by $H \psi \equiv \exists m n$. $D_{m} \psi \wedge \rho(n, m) \wedge \beta^{n}$. Then $\rho(n, m) \Leftrightarrow A_{n}\left(H \gamma^{m}\right)$. Proof. Since $\rho$ respects $\nLeftarrow$ on the right,

$$
\begin{aligned}
H \gamma^{m} & =\exists m^{\prime} n . D_{m^{\prime}} \gamma^{m} \wedge \rho\left(n, m^{\prime}\right) \wedge \beta^{n} \\
& =\exists m^{\prime} n .\left(m^{\prime} \ll m\right) \wedge \rho\left(n, m^{\prime}\right) \wedge \beta^{n} \\
& =\exists n \cdot \rho(n, m) \wedge \beta^{n} .
\end{aligned}
$$

Then, since $\rho$ also respects $\nVdash$ on the left and $A_{n}$ preserves the join, which is directed because $\rho$ respects 0 and + ,

$$
\begin{aligned}
A_{n}\left(H \gamma^{m}\right) & \Leftrightarrow A_{n}\left(\exists n^{\prime} \cdot \rho\left(n^{\prime}, m\right) \wedge \beta^{n^{\prime}}\right) \\
& \Leftrightarrow \exists n^{\prime} \cdot \rho\left(n^{\prime}, m\right) \wedge A_{n} \beta^{n^{\prime}} \\
& \Leftrightarrow \exists n^{\prime} \cdot \rho\left(n^{\prime}, m\right) \wedge n \preccurlyeq n^{\prime} \Leftrightarrow \rho(n, m) .
\end{aligned}
$$

Lemma 16.7. $\operatorname{id}_{n}^{m} \Leftrightarrow A_{n}\left(\mathrm{id} \beta^{m}\right) \Leftrightarrow(n \prec m) \Leftrightarrow \widehat{\mathcal{E}}_{n}^{m}$.

Proof. The relationship with $\mathcal{E}$ follows from Lemma 14.5. The unit laws were given by Lemma 16.5 $c f$. the Karoubi completion, which splits idempotents in any category. 
Lemma 16.8. ${\widehat{K} \cdot H_{n}^{k}}_{k} \Leftrightarrow m . \widehat{K}_{m}^{k} \wedge \widehat{H}_{n}^{m}$.

Proof.

$$
\begin{array}{rlr}
\widehat{K \cdot H}_{n}^{k} & \Leftrightarrow A_{n}\left(H\left(K \epsilon^{k}\right)\right) & \\
& \Leftrightarrow A_{n}\left(\exists m n^{\prime} \cdot D_{m}\left(K \epsilon^{k}\right) \wedge \widehat{H}_{n^{\prime}}^{m} \wedge \beta^{n^{\prime}}\right) & \text { Lemma 16.2 } \\
& \Leftrightarrow \exists m n^{\prime} . D_{m}\left(K \epsilon^{k}\right) \wedge \widehat{H}_{n^{\prime}}^{m} \wedge A_{n} \beta^{n^{\prime}} & \\
& \Leftrightarrow \exists m n^{\prime} . D_{m}\left(K \epsilon^{k}\right) \wedge \widehat{H}_{n^{\prime}}^{m} \wedge\left(n \prec n^{\prime}\right) & \\
& \Leftrightarrow \exists m . D_{m}\left(K \epsilon^{k}\right) \wedge \widehat{H}_{n}^{m} & \text { Lemma [16.5 } \\
& \Leftrightarrow \exists m . \widehat{K}_{m}^{k} \wedge \widehat{H}_{n}^{m}, & \operatorname{def} K
\end{array}
$$

although $\Leftarrow$ is actually $\Leftrightarrow$ as we have an $\vee$-basis. (We draw attention to this because I uses the natural $\wedge$-basis on $\mathbb{R}$, and therefore the weaker result about composition.)

Theorem 16.9. HS (Notation 5.13) is equivalent to the category whose

(a) objects are abstract bases $(N, 0,1,+, \star, \gtrless)$ (Definition 14.1);

(b) morphisms are $\rho(n, m)$ satisfying the conditions in Lemma 16.6;

(c) identity is ;

(d) composition is relational.

The definition of $\mathrm{HS}$ in $[\mathrm{A}$ was essentially taken from Hayo Thielecke's work on "computational effects" Thi97, which was in turn based on the Kleisli category for the monad. It was therefore motivated by more syntactic considerations than ours. From a semantic point of view, it would have been more natural to have split the idempotents. In the classical models, the category would then be the opposite of that of all continuous (but not necessarily distributive) lattices and Scott-continuous maps ( $c f$. Example 12.6(b)]. In this result, we would drop the $\preccurlyeq+$ and $\preccurlyeq \star$ rules from Definition 14.1 of an abstract basis.

In order to characterise first class maps, by Corollary 10.3 we have to consider preservation of the lattice connectives, $c f$. Lemmas 15.3f. For this, the target object $Y$ must have a lattice basis.

Lemma 16.10. $H \top=\top$ iff $\widehat{H}_{n}^{1} \Leftrightarrow(n \prec 1)$, and $H \perp=\perp$ iff $\widehat{H}_{n}^{0} \Leftrightarrow(n \prec 0)$.

Proof. If $H \top=\top$ then $\widehat{H}_{n}^{1} \equiv A_{n}\left(H \gamma^{1}\right) \equiv A_{n}(H \top) \Leftrightarrow A_{n} \top \equiv A_{n} \beta^{1} \equiv(n \prec 1)$ by Notation [16.1 Definition 6.6] and Notation 11.1.

Conversely, $H \top \equiv H \gamma^{1} \equiv \exists n . \widehat{H}_{n}^{1} \wedge \beta^{n} \Leftrightarrow \exists n . A_{n} \top \wedge \beta^{n} \equiv \top$ by Definition 6.6. Lemma 16.5 and Definition 6.2

We may substitute $\perp$ and 0 for $T$ and 1 in the same argument.

Similarly we are able on this occasion to handle $\wedge$ and $\vee$ simultaneously (Remark 4.15).

Lemma 16.11. $H(\phi \odot \psi)=H \phi \odot H \psi$ iff $\widehat{H}_{n}^{s \odot t} \Leftrightarrow \exists m p . \widehat{H}_{m}^{s} \wedge \widehat{H}_{p}^{t} \wedge(n \prec m \odot p)$, both when $\odot$ is $\wedge$ or $\star$ and when it is $\vee$ or + . 
Proof. If $H(\phi \odot \psi)=H \phi \odot H \psi$ then

$$
\begin{array}{rlr}
\widehat{H}_{n}^{s \odot t} & \Leftrightarrow A_{n}\left(H \beta^{s \odot t}\right) \Leftrightarrow A_{n}\left(H\left(\beta^{s} \odot \beta^{t}\right)\right) & \\
& \Leftrightarrow A_{n}\left(H \beta^{s} \odot H \beta^{t}\right) & \text { hypothesis } \\
& \Leftrightarrow \exists m p . A_{m}\left(H \beta^{s}\right) \wedge A_{p}\left(H \beta^{t}\right) \wedge(n \preccurlyeq m \odot p) & \text { Lemma 11.9 } \\
& \Leftrightarrow \exists m p . \widehat{H}_{m}^{s} \wedge \widehat{H}_{p}^{t} \wedge(n \preccurlyeq m \odot p) &
\end{array}
$$

Conversely, using distributivity,

$$
\begin{array}{rlr}
H \phi \odot H \psi & =\left(\exists m u . D_{m} \phi \wedge \widehat{H}_{u}^{m} \wedge \beta^{u}\right) \odot\left(\exists p v . D_{p} \psi \wedge \widehat{H}_{v}^{p} \wedge \beta^{v}\right) & \text { Lemma 16.2] } \\
& =\exists m p u v . D_{m} \phi \wedge D_{p} \psi \wedge \widehat{H}_{u}^{m} \wedge \widehat{H}_{v}^{p} \wedge \beta^{u \odot v} & \text { Lemma 4.17] } \\
& =\exists k m p u v . D_{m} \phi \wedge D_{p} \psi \wedge \widehat{H}_{u}^{m} \wedge \widehat{H}_{v}^{p} \wedge(k \prec u \odot v) \wedge \beta^{k} & \text { L. 11.4 } \\
& =\exists k m p . D_{m} \phi \wedge D_{p} \psi \wedge \widehat{H}_{k}^{m \odot p} \wedge \beta^{k} & \text { hypothesis } \\
& =\exists n k m p . D_{m} \phi \wedge D_{p} \psi \wedge(n \prec m \odot p) \wedge \widehat{H}_{k}^{n} \wedge \beta^{k} & \text { Lemma 16.5] } \\
& =\exists n k . D_{n}(\phi \odot \psi) \wedge \widehat{H}_{k}^{n} \wedge \beta^{k} & \text { Lemma 11.9 } \\
& =H(\phi \odot \psi) & \text { Lemma 16.2] }
\end{array}
$$

Definition 16.12. An abstract matrix is a binary relation $\widehat{H}_{n}^{m}$ such that

$$
\begin{aligned}
& \widehat{H}_{0}^{m} \quad \frac{\widehat{H}_{n}^{m} \widehat{H}_{p}^{m}}{{\widehat{H_{n+p}^{m}}}_{n}^{m}} \quad \frac{n^{\prime} \preccurlyeq X n}{\widehat{H}_{n}^{m} \quad m \preccurlyeq{ }_{Y} m^{\prime}} \quad \frac{\widehat{H}_{n^{\prime}}^{m^{\prime}}}{\overline{\hat{H}_{n^{\prime}}^{m^{\prime}}}}
\end{aligned}
$$

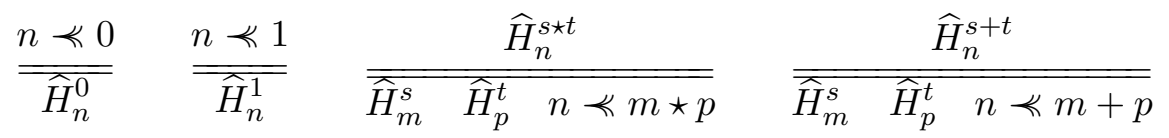

Theorem 16.13. $\mathcal{S}$ is equivalent to the category whose

(a) objects are abstract lattice bases $(N, 0,1,+, \star, \gtrless)$;

(b) morphisms are abstract matrices;

(c) identity is «;

(d) composition is relational.

Jung and Sünderhauf characterised continuous functions between stably locally compact spaces in a similar way JS96.

Remark 16.14. We shall speculate on the possible computational applications of matrices for continuous functions in the next section, but let's say something here about the analogy with linear algebra that we have used. Really, this has been much more useful that we had any right to expect, since neither $\mathcal{S}$ nor $\mathrm{H} \mathcal{S}$ (the "first" and "second class" maps) is a symmetric monoidal closed category.

However, by Theorem 9.11 stably locally compact objects and the $\widehat{A}: X \rightarrow Y$ for which $A$ preserves $\top$ and $\wedge$ do define such a category. In fact, they provide a model of linear logic with involutive negation and an "of course" operator given by the Smyth powerdomain $\mathcal{P}^{\sharp}$ (Example 12.13):

Proposition 16.15. Let $X$ be a stably locally compact space. The $\mathrm{HS}$-maps $\widehat{A}: \Gamma \longrightarrow \times X$ for which $A: \Sigma^{X} \rightarrow \Sigma^{\Gamma}$ preserves $\top$ and $\wedge$ correspond bijectively and naturally to $\mathcal{S}$-maps $\Gamma \rightarrow \mathcal{P}^{\sharp} X$. 
Proof. Both correspond to terms $\Gamma \vdash \xi \equiv \lambda n . A \beta^{n}: \Sigma^{N}$ that are rounded filters $(\wedge-$ homomorphisms) for $\prec_{X}$ or ideals for $\succcurlyeq_{X}$.

Since they also preserve directed joins, such $A$ are known as preframe homomorphisms; for more on their relationship to the Smyth powerdomain, see [Vic88, 11.2.5] and JKM01. For a similar investigation of join-preserving maps and the Hoare or lower powerdomain $\mathcal{P}^{b} X$ in ASD, presumably we would first need to identify the "stably locally overt" objects to which the analogous construction may be applied.

\section{Relating the Classical AND term MOdels}

We promised to translate each step of the logical development into topological language, but we haven't done this since the end of Section 7 . We shall now show how the "coding" provides the link between the classical and computational models (Remark 3.1), and then how abstract matrices themselves describe exact computation for the reals and locally compact objects in general.

Remark 17.1. On the one hand, we know from the classical proof for LKSp in $[\mathrm{A}$, Theorem 5.12] and the intuitionistic one for LKLoc in [B. Theorem 3.11] that these categories are models of the calculus in Sections 3 4 , i.e. that there are interpretation functors $\llbracket-\rrbracket: \mathcal{S} \rightarrow$ LKSp and $\llbracket-\rrbracket: \mathcal{S} \rightarrow$ LKLoc. In the light of Theorem 8.11 that every object of $\mathcal{S}$ is a $\Sigma$-split subobject of $\Sigma^{\mathbb{N}}$, the converse part of Theorem 7.14 provides another proof in the localic setting.

In this paper we have sought the "inverse" of this functor. Since the classical models are of course richer, they have to be constrained in order to obtain something equivalent to the computational one (Remark 3.1). This constraint was in the form of a computable basis, as in Definitions 1.5] and 2.3. Nevertheless, as we saw in the case of $\mathbb{R}$ (Example 1.4), such bases may already be familiar to us from traditional considerations.

There is no need to verify the consistency conditions that we set out in Sections 11 and 14. They follow automatically from the existence of the classical space, which serves as a reference as in Remark 1.7 So long as $\star,+$ and $\preccurlyeq$ are defined by programs, which can be translated into our $\lambda$-calculus, we already have an abstract basis.

Examples 17.2. At this point let us recall the various ways in which a lattice basis can be defined on a locally compact sober space or locale.

(a) In a stably locally compact sober space (which is, in particular, compact in the global sense), we may choose a sublattice of compact subspaces $K^{n}$ and corresponding sublattice of open ones $U^{n}$, such that $U^{n} \subset K^{n}$ and the basis expansion is satisfied. Then the indexing set $N$, together with the operations + and $\star$ on codes corresponding to unions and intersections of open-compact pairs, and the relation $n \preccurlyeq m$ given by $K^{n} \subset U^{m}$, define an abstract basis, so long as these operations are computable. In the corresponding lattice filter basis in the $\lambda$-calculus, $\beta^{n}$ and $A_{n}$ classify $U^{n}$ and the Scott-open filter $\mathcal{F}_{n} \equiv\left\{V \mid K^{n} \subset V\right\}$.

(b) In a compact Hausdorff space, the compact subspaces $K^{n}$ are the complements of open subspaces $V_{n}$, which may be chosen from the same sublattice as the $U^{n}$, but with $U^{n} \pitchfork V_{n}$.

(c) In particular, finite unions of open or of closed rational intervals provide this structure for the closed real unit interval $[0,1]$. 
(d) $\mathbb{R}$ is not globally compact, though binary intersections of compact subspaces are compact. The lattice basis may be defined in the same way as for $[0,1]$, with the single exception of $A_{1} \equiv \lambda \phi . \perp$, which does not preserve $\top$.

(e) Let $(N, \triangleleft)$ be a recursively enumerable directed interpolative relation (Definition 12.1). Then Theorem 12.11 defines an object in $\mathcal{S}$, whose classical interpretation is the continuous dcpo of rounded ideals of $(N, \triangleleft)$; it is algebraic iff $\triangleleft$ is reflexive.

(f) The reflexive order $\preccurlyeq$ defined from any imposed distributive lattice $(N, 0,1,+, \star)$ by Definition 13.10 satisfies the conditions on $\preccurlyeq$ for an abstract basis, and so defines an object of $\mathcal{S}$ whose classical interpretation is the coherent space whose compact open subspaces are indexed by $N$.

(g) Given a locally compact locale, we choose a sublattice $N$ that provides a basis for the corresponding continuous distributive lattice $L$, with inclusion $\beta^{(-)}: N \rightarrow L$. Then define $(n \ll m) \equiv\left(\beta^{n} \ll \beta^{m}\right)$. This is a lattice basis, but only a filter basis in the stably locally compact case.

(h) Finally, in the case of a non-stably locally compact sober space, we only have a $U$ semilattice $N$ of compact subspaces $K^{n}$, and therefore a (filter) $\vee$-basis $\left(\beta^{n}, A_{n}\right)$. Remark 8.7 turned this into a lattice basis $\left(\beta^{\ell}, A_{\ell}\right)$ indexed by $\operatorname{Fin}(N)$, by defining

$$
\beta^{\ell} x \equiv \forall n \in \ell . x \in U^{n} \text { and } A_{\ell} \phi \equiv \exists n \in \ell . K^{n} \subset V,
$$

where $\phi$ classifies $V$ as usual. Then $\beta^{\ell}$ simply classifies the intersection of the basic open subspaces as in the stably locally compact case, but $A_{\ell}$ is a logical disjunction, not a union of subspaces, $c f$. Lemma 5.7(e) Then

$$
\ell \prec \ell^{\prime} \Leftrightarrow \exists n \in \ell . \forall m \in \ell^{\prime} . K^{n} \subset U^{m},
$$

but this is not a filter basis.

Remark 17.3. Section 14 showed that the abstract basis is inter-definable with the nucleus $\mathcal{E}$. These are interpreted both in the computational model $\mathcal{S}$ and in the classical ones LKSp and LKLoc. This means that the idempotent $\llbracket \mathcal{E} \rrbracket$ on $\Upsilon K \mathbb{N}$ is the one that defines the $\Sigma$-split embedding of the original space in $\mathcal{P}(\mathbb{N})$, as in Theorems 7.8 and 7.14 .

Hence any classically defined locally compact sober space or locale that has a computable basis may be "imported" into abstract Stone duality as an object, whose interpretation in the classical model is homeomorphic to the given space. Summing this up diagrammatically,

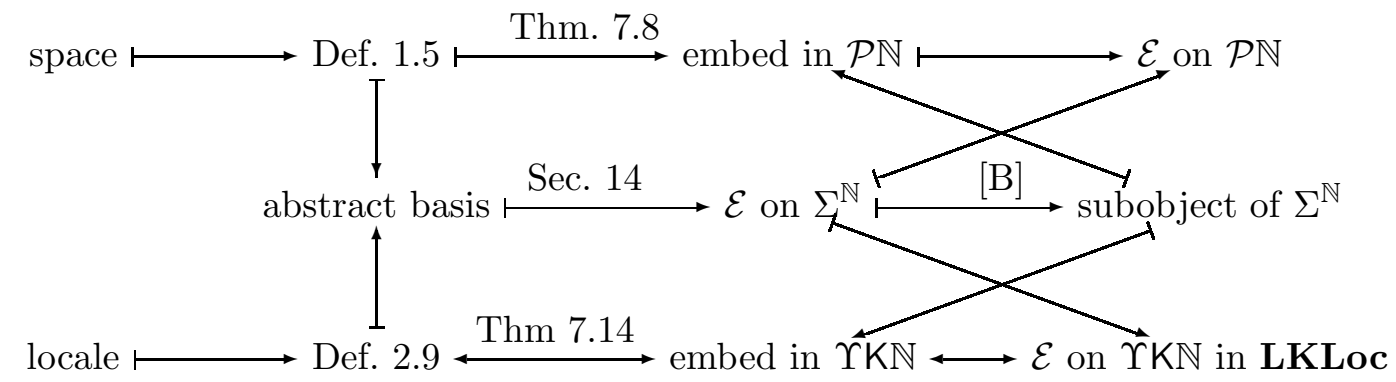

Remark 17.4. Having fixed computational bases for two classically defined spaces or locales, $X$ and $Y$, we may look at continuous functions $f: X \rightarrow Y$. By the basis property, any such map is determined by the relation

$$
f K^{n} \subset V^{m}
$$


as $n$ and $m$ range over the bases for $X$ and $Y$ respectively. If this relation is recursively enumerable (Definition 1.6) then the corresponding program may be translated into our $\lambda$ calculus. Just as we saw for abstract bases, the resulting term satisfies Definition 16.12 for an abstract matrix, because its interpretation agrees with a continuous function. Between the objects whose denotations are $X$ and $Y$ there is therefore a term whose denotation is $f$.

In particular, computationally equivalent bases for the same space give rise to an isomorphism between the objects of $\mathcal{S}$. In the case of morphisms, extensionally equivalent terms give rise to the same $\left(f K^{n} \subset V^{m}\right)$-relations, and therefore to the same continuous functions. However, programs may be extensionally equivalent for some deep mathematical reason, or as a result of the stronger logical principles in the classical situation, without being provably equivalent within our calculus. This is the reason why we required the computable aspects of the definitions in the Introduction to be accompanied by actual programs.

This completes the proof of our main result:

Theorem 17.5. Abstract Stone duality, i.e. the free model $\mathcal{S}$ of the axioms in Sections 3 and 4 is equivalent to the category of computably based locally compact locales and computably continuous functions.

Remark 17.6. An obvious lacuna in this result arises from the difference between sober spaces and locales: we are relying on the axiom of choice within the classical models to say that the two are the same. Recall [Joh82, Theorem VII 4.3] that, using excluded middle, the crucial requirement is to find, for any $\phi \not \leq \psi: L$ in a distributive continuous lattice, (a) some (Scott open) filter $A: \Sigma^{L}$ for which $A \phi \Leftrightarrow \top$ but $A \psi \Leftrightarrow \perp$, and then

(b) using Zorn's lemma, enlarge $A$ to a maximal such filter $P$, which is prime.

For stage (a), we would like to show, more generally, that every object (which we know carries a lattice basis) also has a filter $\vee$-basis. The idea, due to Jimmie Lawson $\mathrm{GHK}^{+} 80$, $\S I$ 3.3], is to iterate the interpolation property $\mathrm{G}_{-}$. This can be shown with the aid of a weaker choice principle that merely extracts a total function from a non-deterministic one.

For stage (b), since any space has a $\mathbb{N}$-indexed lattice basis, we only need to consider $\Sigma^{\mathbb{N}}$, and not general distributive continuous lattices. The filter $A$ provides a rounded filter (semilattice homomorphism) $\xi_{0}: \Sigma^{\mathbb{N}}$ such that $I \phi \xi \Leftrightarrow \top$ but $I \psi \xi \Leftrightarrow \perp$, which we enlarge to a rounded lattice homomorphism $\xi$ (Lemmas 15.2f) with the same values on $\phi$ and $\psi$. To do this, we may replace Zorn's Lemma by an argument that uses excluded middle but not Choice: we build up $\xi$ from $\xi_{0}$ by adding each number $0,1,2, \ldots$ in turn if it generates a proper filter.

Remark 17.7. Let's review what we've achieved by way of a type theory for topology.

(a) The original idea of abstract Stone duality was that the non-computable unions could be eliminated from general topology by expressing the category of "frames" by a monadic adjunction over its opposite category of "spaces" rather than over sets.

(b) Beck's theorem says that this is equivalent to the, perhaps less friendly, condition that the functor $\Sigma^{(-)}$reflect invertibility and "create $\Sigma$-split coequalisers".

(c) In $[\mathrm{B}$ we saw that the latter can be interpreted as (certain) subobjects, and that the data for such subobjects could be encapsulated in a $\operatorname{single~morphism~} \mathcal{E}$, called a "nucleus".

(d) This was further developed, in Section 8 of that paper, into a $\lambda$-calculus similar to comprehension in set theory. However, the data defining a subobject remained arcane: 
terms satisfying the equation defining a nucleus could only be found with considerable expert ingenuity.

(e) In Theorem 10.10 of this paper we reformulated the $\lambda$-equation in a much simpler way using the lattice connectives.

(f) The notion of abstract basis in this paper puts the construction within the grasp of anyone who has a knowledge of open and compact subspaces in topology.

Remark 17.8. Let us consider the computational meaning of the matrix $\widehat{H}_{n}^{m}$ when $H \equiv \Sigma^{f}$ for some continuous function $f: \mathbb{R} \rightarrow \mathbb{R}$. In order to have a lattice basis, the indices $n$ and $m$ must range over (finite) unions of intervals, although by Lemma 16.3 $n$ need only denote a single closed interval. The matrix therefore encodes the predicate

$$
f\left[x_{0} \pm \delta\right] \subset \bigcup_{j}\left(y_{j} \pm \epsilon_{j}\right)
$$

as an ASD term of type $\Sigma$, the union being finite. Suppose that we have a real input value $x$ that we know to lie in the interval $\left[x_{0} \pm \delta\right]$, and we require $f(x)$ to within $\epsilon$.

We substitute the rational values $x_{0}, \delta$ and $\epsilon_{j} \equiv \epsilon$ in the predicate, leaving $\left(y_{j}\right)$ indeterminate. Recall from Remark [3.5 and [A, Remark 11.3] that any such term may be translated into a $\lambda$-PROLOG program. Such a program permits substitution of values for any subset of the free variables, and is executed by resolving unification problems, which result in values of (or at least constraints on) the remaining variables. In this case, we obtain (nondeterministically) some finite set $\left(y_{j}\right)$.

In the language of real analysis, we are seeking to cover the compact interval $f\left[x_{0} \pm \delta\right]$ with (finitely many) open intervals of size $\epsilon$, centred on the $y_{j}$. The Wilker property (Lemma 16.11) then provides

$$
\left[x_{0} \pm \delta\right] \subset \bigcup_{j}\left(x_{j} \pm \delta^{\prime}\right) \quad \text { with } \quad f\left[x_{j} \pm \delta^{\prime}\right] \subset\left(y_{j} \pm \epsilon\right)
$$

Responsibility now passes back to the supplier of the input value $x$ to choose which of the $x_{j}$ is nearest, and the corresponding $y_{j}$ is the required approximation to the result $f(x)$. This discussion is taken up again in I, J].

Remark 17.9. This illustrates the way in which we would expect to use abstract Stone duality for computations with objects such as $\mathbb{R}$ that we regard, from a mathematical point of view, as "base types" (though of course only $\mathbf{1}, \mathbb{N}$ and $\Sigma$ are actually base types of our $\lambda$-calculus). Where higher types, such as continuous or differentiable function-spaces, can be shown to be locally compact, they too have bases and matrices, but it would be an example of the mis-use of normalisation theorems (Remark 8.12) to insist on reducing everything to matrix form. We would expect to use higher-type $\lambda$-terms of our calculus to encode higher-order features of analysis, for example in the calculus of variations. Of course, much preliminary work with $\mathbb{R}$ itself needs to be done before we see what can be done in such subjects [I, [J].

Remark 17.10. The manipulations that we have done since introducing the abstract waybelow relation have all required lattice bases. The naturally occurring basis on an object such as $\mathbb{R}$, on the other hand, is often just an $\wedge$-basis. This didn't matter in Sections 14 16. as they were only concerned with the theoretical issue of the consistency of the abstract basis. We have just seen, however, that the "matrices" in Section 16 encapsulate actual 
computation, in which the base types are those of the indices of the bases. The lists used in Lemma 8.4 would then be a serious burden.

There is a technical issue here that is intrinsic to topology. In locale theory, which is based on an algebraic theory of finite meets distributing over arbitrary unions of "opens", it is often necessary to specify when two such expressions are equal, which may be reduced to the question of when an intersection is contained in a union of intersections. This coverage relation is an important part of the technology of locale theory [Joh82, Section II 2.12], whilst it was chosen as the focus of the axiomatisation of Formal Topology.

Remark 17.11. The question of whether, using abstract Stone duality, we can develop a technically more usable approach than these warrants separate investigation, led by the examples. Since, in a locally compact object, we may consider coverages of compact subobjects, the covering families of open subobjects need only be finite. Jung, Kegelmann and Moshier have exploited this idea to develop a Gentzen-style sequent calculus [JKM99.

As this finiteness comes automatically, maybe we don't need to force it by using lists. To put this another way, as the lists act disjunctively, we represent them by their membership predicates (Remark 8.5). That is, we replace the matrix $\widehat{H}_{m}^{n}$ with the predicate

$$
m: M, \xi: \Sigma^{N} \vdash A_{m} \cdot H\left(\lambda y \cdot \exists n \cdot \xi n \wedge \beta^{n} y\right): \Sigma,
$$

where $N$ need no longer have + . In Remark 17.8 above, we could add the constraint that $\xi$ only admits intervals of size $<\epsilon$.

Remark 17.12. Another striking feature of the matrix description is that it reduces the topological theory to an entirely discrete one. The latter may be expressed in an arithmetic universe, which is a category with finite limits, stable disjoint coproducts, stable effective quotients of equivalence relations and a List functor $[\mathrm{E}$.

Once again, we need to see this normalisation theorem in reverse. It appears that any arithmetic universe may conversely be embedded as the full subcategory of overt discrete objects in a model of abstract Stone duality. Example 12.6(d) shows that we can do this by modelling a much simpler structure than that of abstract bases and matrices for general locally compact spaces. The differences in logical strength amongst models of ASD are therefore measured by the overt discrete objects that they contain.

Such a construction would enable topological, domain-theoretic and $\lambda$-calculus reasoning to be applied to problems in discrete algebra and logic. Topologically, it would strongly vindicate Marshall Stone's dictum, always topologise, whilst computationally it would provide continuation-passing translations of discrete problems, and of type theories for inductive types.

\section{REFERENCES}

[Bar81] Henk Barendregt. The Lambda Calculus: its Syntax and Semantics. Number 103 in Studies in Logic and the Foundations of Mathematics. North-Holland, 1981. Second edition, 1984.

[Bou66] Nicolas Bourbaki. Topologie Générale. Hermann, 1966. English translation, "General Topology", distrubuted by Springer-Verlag, 1989.

[CS88] John Horton Conway and Neil Sloane. Sphere Packings, Lattices and Groups. Number 290 in Grundlehren der Mathematischen Wissenschaften. Springer-Verlag, 1988.

$\left[\mathrm{GHK}^{+} 80\right]$ Gerhard Gierz, Karl Heinrich Hoffmann, Klaus Keimel, Jimmie Lawson, Michael Mislove, and Dana Scott. A Compendium of Continuous Lattices. Springer-Verlag, 1980. Second edition, Continuous Lattices and Domains, published by Cambridge University Press, 2003. 
[Har40] G. H. Hardy. A Mathematician's Apology. Cambridge University Press, 1940. Frequently reprinted.

[HL78] Karl Hofmann and Michael Lawson. Spectral theory of continuous lattices. Transactions of the American Mathematical Society, 246:285-310, 1978.

[HM81] Karl Hofmann and Michael Mislove. Local compactness and continuous lattices. In Bernhard Banaschewski and Rudolf-Eberhard Hoffmann, editors, Continuous Lattices, volume 871 of Springer Lecture Notes in Mathematics, pages 209-248, 1981.

[JKM99] Achim Jung, Mathias Kegelmann, and Andrew Moshier. Multi lingual sequent calculus and coherent spaces. Fundamenta Informaticae, 37(4):369-412, 1999.

[JKM01] Achim Jung, Mathias Kegelmann, and Andrew Moshier. Stably compact spaces and closed relations. Electronic Notes in Theoretical Computer Science, 45, 2001. MFPS 17.

[Joh82] Peter Johnstone. Stone Spaces. Number 3 in Cambridge Studies in Advanced Mathematics. Cambridge University Press, 1982.

[Joh84] Peter Johnstone. Open locales and exponentiation. Contemporary Mathematics, 30:84-116, 1984.

[Joh85] Peter Johnstone. Vietoris locales and localic semi-lattices. In R.-E. Hoffmann and K. H. Hoffmann, editors, Continuous Lattices and their Applications, number 101 in Pure and Applied Mathematics, pages 155-180. Marcel Dekker, 1985.

[JS96] Achim Jung and Philipp Sünderhauf. On the duality of compact vs. open. In S. Andima, R.C. Flagg, G. Itzkowitz, P. Misra, Y. Kong, and R.D. Kopperman, editors, Papers on general topology and applications: Eleventh Summer Conference at University of Southern Maine, volume 806 of Annals of the New York Academy of Sciences, pages 214-230, 1996.

[Jun90] Achim Jung. The classification of continuous domains. In Logic in Computer Science, pages 35-40. IEEE Computer Society Press, 1990.

[Kel55] John Kelley. General Topology. Van Nostrand, 1955. Reprinted by Springer-Verlag, Graduate Texts in Mathematics, 27, 1975.

[Plo77] Gordon Plotkin. LCF considered as a programming language. Theoretical Computer Science, 5:223-255, 1977.

[Sco70] Dana Scott. Outline of a mathematical theory of computation. In 4th Annual Princeton Conference on Information Sciences and Systems, pages 169-176, 1970. Superseded by Oxford technical report PRG-2.

[Sco82] Dana Scott. Domains for denotational semantics. In M. Nielson and E. M. Schmidt, editors, Automata, Languages and Programming: Proceedings 1982. Springer-Verlag, 1982. Lecture Notes in Computer Science 140.

[Smy77] Michael Smyth. Effectively given domains. Theoretical Computer Science, 5:257-274, 1977.

[Tay91] Paul Taylor. The fixed point property in synthetic domain theory. In Gilles Kahn, editor, Logic in Computer Science 6, pages 152-160. IEEE, 1991.

[Tay99] Paul Taylor. Practical Foundations of Mathematics. Number 59 in Cambridge Studies in Advanced Mathematics. Cambridge University Press, 1999.

[Thi97] Hayo Thielecke. Categorical Structure of Continuation Passing Style. PhD thesis, University of Edinburgh, 1997. Also available as technical report ECS-LFCS-97-376.

[Vic88] Steven Vickers. Topology Via Logic, volume 5 of Cambridge Tracts in Theoretical Computer Science. Cambridge University Press, 1988.

[Wil70] Peter Wilker. Adjoint product and hom functors in general topology. Pacific Journal of Mathematics, 34:269-283, 1970.

The papers on abstract Stone duality may be obtained from

$$
\text { WWW.cs.man.ac.uk/ pt/ASD }
$$

[A] Paul Taylor, Sober spaces and continuations Theory and Applications of Categories, 10(12):248-299, 2002.

[B] Paul Taylor, Subspaces in abstract Stone duality Theory and Applications of Categories, 10(13):300-366, 2002.

[C] Paul Taylor, Geometric and higher order logic using abstract Stone duality Theory and Applications of Categories, 7(15):284-338, 2000. 
[D] Paul Taylor, Non-Artin gluing in recursion theory and lifting in abstract Stone duality. 2000.

[E] Paul Taylor, Inside every model of Abstract Stone Duality lies an Arithmetic Universe Electronic Notes in Theoretical Computer Science 416, Elsevier, 2005.

[F] Paul Taylor, Scott domains in abstract Stone duality. March 2002.

[G-] Paul Taylor,

Local compactness and the Baire category theorem in abstract Stone duality Electronic Notes in Theoretical Computer Science 69 Elsevier, 2003.

$[\mathrm{H}] \quad$ Paul Taylor, An elementary theory of various categories of spaces and locales November 2004.

[I] Andrej Bauer and Paul Taylor, The Dedekind reals in abstract Stone duality Computability and Complexity in Analysis, Kyoto, August 2005.

$[\mathrm{J}] \quad$ Paul Taylor, A $\lambda$-calculus for real analysis Computability and Complexity in Analysis, Kyoto, August 2005.

\section{ACKNOWLEDGEMENTS}

This paper evolved from [G], which included roughly Sections 5 [8] and 11 of the present version. It was presented at Category Theory and Computer Science $\mathbf{9}$ in Ottawa on 17 August 2002, and at Domains Workshop 6 in Birmingham a month later. The characterisation of locally compact objects using effective bases (Section 6) had been announced on categories on 30 January 2002.

The earlier version also showed that any object has a filter basis, and went on to prove Baire's category theorem, that the intersection of any sequence of dense open subobjects (of any locally compact overt object) is dense. These arguments were adapted from the corresponding ones in the theory of continuous lattices $\mathrm{GHK}^{+} 80$, Sections I 3.3 and 3.43].

I would like to thank Andrej Bauer, Martín Escardó, Peter Johnstone, Achim Jung, Jimmie Lawson, Graham White and the CTCS and LMCS referees for their comments. Graham White has given continuing encouragement throughout the abstract Stone duality project, besides being an inexhaustible source of mathematical ideas.

This research is now supported by UK EPSRC project GR/S58522, but this funding was obtained in part on the basis of the work in this paper. Apart from[5.19 12.13 12.14 15.3 15.5] 16.15] and 17.6 the work here was carried out during a period of unemployment, supported entirely from my own savings. However, I would have been unable to do this without the companionship and emotional support of my partner, Richard Symes.

This work is licensed under the Creative Commons Attribution-NoDerivs License. To view a copy of this license, visit http://creativecommons.org/licenses/by-nd/2.0/ or send a letter to Creative Commons, 559 Nathan Abbott Way, Stanford, California 94305, USA. 